\title{
THE CARINA PROJECT. IX. ON HYDROGEN AND HELIUM BURNING VARIABLES
}

\author{
G. Coppola ${ }^{1}$, M. Marconi ${ }^{1}$, P. B. Stetson ${ }^{2}$, G. Bono $^{3,4}$, V. F. Braga ${ }^{3,4}$, V. Ripepi ${ }^{1}$, M. Dall'Ora ${ }^{1}$, I. Musella ${ }^{1}$,

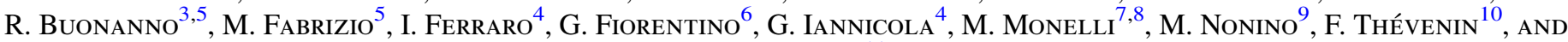 \\ A. R. WALKER ${ }^{11}$ \\ ${ }^{1}$ INAF-Osservatorio Astronomico di Capodimonte, Via Moiariello 16, I-80131 Napoli, Italy; marcella@oacn.inaf.it \\ ${ }^{2}$ National Research Council, 5071 West Saanich Road, Victoria, BC V9E 2E7, Canada \\ ${ }^{3}$ Dipartimento di Fisica, Universitá di Roma Tor Vergata, Via della Ricerca Scientifica 1, I-00133 Roma, Italy \\ ${ }^{4}$ INAF-Osservatorio Astronomico di Roma, Via Frascati 33, I-00040 Monte Porzio Catone, Italy \\ 5 INAF-Osservatorio Astronomico di Teramo, via M. Maggini, I-64110, Teramo, Italy \\ ${ }^{6}$ INAF-Osservatorio Astronomico di Bologna, via Ranzani 1, I-40127, Bologna, Italy \\ ${ }^{7}$ Instituto de Astrofísica de Canarias, Calle Via Lactea s/n, E-38205 La Laguna, Tenerife, Spain \\ 8 Departmento de Astrofísica, Universidad de La Laguna, E-38200 La Laguna, Tenerife, Spain \\ 9 INAF-Osservatorio Astronomico di Trieste, via G. B. Tiepolo 11, I-40131 Trieste, Italy \\ ${ }^{10}$ Laboratoire Lagrange, Universit Cte d'Azur, Observatoire de la Cte d'Azur, CNRS, Blvd de l'Observatoire, CS 34229, F-06304 Nice cedex 4, France \\ ${ }^{11}$ Cerro Tololo Inter-American Observatory, National Optical Astronomy Observatory, Casilla 603, La Serena, Chile \\ Received 2015 July 7; accepted 2015 September 8; published 2015 November 17
}

\begin{abstract}
We present new multiband $(U B V I)$ time-series data of helium burning variables in the Carina dwarf spheroidal galaxy. The current sample includes 92 RR Lyrae-six of them are new identifications-and 20 Anomalous Cepheids, one of which is new identification. The analysis of the Bailey diagram shows that the luminosity amplitude of the first overtone component in double-mode variables is located along the long-period tail of regular first overtone variables, while the fundamental component is located along the short-period tail of regular fundamental variables. This evidence further supports the transitional nature of these objects. Moreover, the distribution of Carina double-mode variables in the Petersen diagram $\left(P_{1} / P_{0}\right.$ versus $\left.P_{0}\right)$ is similar to metal-poor globulars (M15, M68), to the dwarf spheroidal Draco, and to the Galactic Halo. This suggests that the Carina old stellar population is metal-poor and affected by a small spread in metallicity. We use trigonometric parallaxes for five field RR Lyrae stars to provide an independent estimate of the Carina distance using the observed reddening free Period-Wesenheit $[\mathrm{PW},(B V)]$ relation. Theory and observations indicate that this diagnostic is independent of metallicity. We found a true distance modulus of $\mu=20.01 \pm 0.02$ (standard error of the mean) \pm 0.05 (standard deviation) mag. We also provided independent estimates of the Carina true distance modulus using four predicted PW relations $(B V, B I, V I, B V I)$ and we found: $\mu=(20.08 \pm 0.007 \pm 0.07) \mathrm{mag}, \mu=(20.06 \pm 0.006 \pm 0.06) \mathrm{mag}$, $\mu=(20.07 \pm 0.008 \pm 0.08) \mathrm{mag}$, and $\mu=(20.06 \pm 0.006 \pm 0.06) \mathrm{mag}$. Finally, we identified more than 100 new SX Phoenicis stars that together with those already known in the literature (340) make Carina a fundamental laboratory for constraining the evolutionary and pulsation properties of these transitional variables.
\end{abstract}

Key words: galaxies: individual (Carina) - Local Group - stars: distances - stars: oscillations (including pulsations) - stars: variables: RR Lyrae

Supporting material: figure set, machine-readable tables

\section{INTRODUCTION}

Dwarf galaxies play a crucial role in several astrophysical and cosmological problems. They outnumber giant stellar systems in the nearby universe and at low redshift (see, e.g., Mateo et al. 1998; McConnachie 2012), but it seems that the number is steadily decreasing at large redshifts (Baldry et al. 2012; Mortlock et al. 2013). The current empirical evidence indicates that the luminosity function does not peak at low-surface brightness systems at redshifts larger than $z=2$ (Bauer et al. 2011; Weinzirl et al. 2011). It is clear that in this context the age distribution of the old stellar populations in nearby dwarf galaxies plays a crucial role in constraining their early formation and evolution (Salvadori et al. 2010). Current empirical evidence indicates that both early- and late-type dwarf galaxies host stellar populations older than $10 \mathrm{Gyr}$. This evidence applies not only to IC10, the Local Group prototype of star-forming galaxies (Sanna et al. 2009), but also to the recently discovered Ultra Faint Dwarf galaxies (Dall'Ora et al. 2006, 2012; Greco et al. 2008; Kuehn et al. 2008; Moretti et al. 2009; Musella et al. 2009, 2012; Clementini et al. 2012;
Garofalo et al. 2013; Fabrizio et al. 2014). Moreover, the comparison between old stellar populations in nearby dwarfs and Galactic Globular clusters does not show, at fixed metal content, any striking difference concerning the age distribution (Monelli et al. 2003; Cole et al. 2007; Bono et al. 2010).

The above empirical evidence suggests that low-mass dark matter halos started assembling baryons at about the same time as the giant halos (Springel et al. 2005; Bauer et al. 2011; Duncan et al. 2014). Detailed constraints on the formation of these systems require detailed investigations into their starformation histories. However, this approach needs deep and accurate color-magnitude diagrams (CMDs) down to limiting magnitudes fainter than the main-sequence turnoff of the old stellar populations (Gallart et al. 2005; Monelli et al. 2010b). In this context variable stars play a crucial role, since they can be easily identified. Moreover, they cover a broad range in age, from a few $100 \mathrm{Myr}$ for Classical Cepheids (e.g., Bono et al. 2005) to a few Gyr for Anomalous Cepheids (AC) (e.g., Caputo 1998; Marconi et al. 2004) to ages older than $10 \mathrm{Gyr}$ for the RR Lyrae stars (RRLs; e.g., Castellani et al. 1991). 
Table 1

Log of Observations

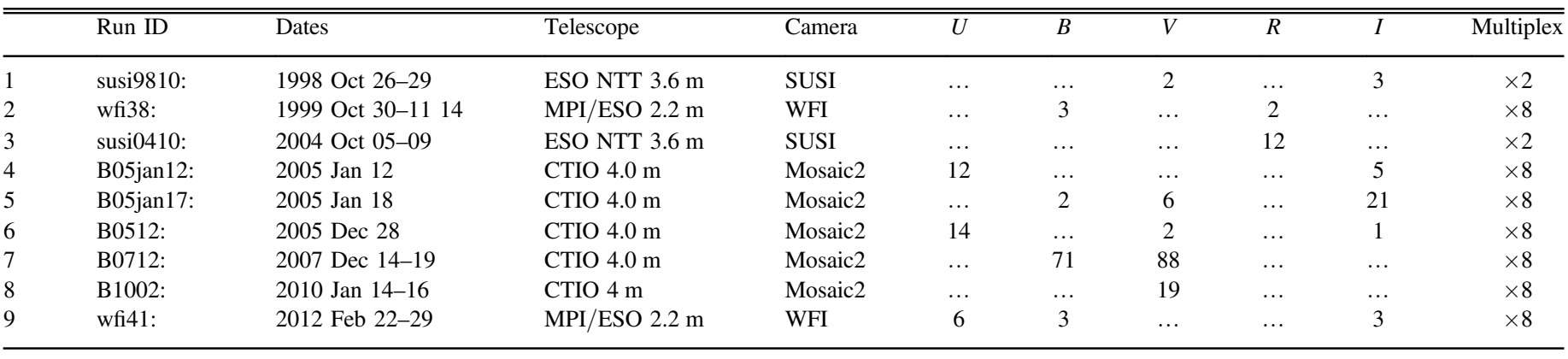

However, for classical Cepheids we have evidence of tight correlation between pulsation and evolutionary age (Matsunaga et al. 2011, 2013). The same outcome does not apply, as noted by the referee, to the other quoted variables. This means that for RRLs we can only provide a lower limit to their individual ages (Bono et al. 2011), while for ACs we can only provide an age interval.

The latter two groups have several distinctive features that make them solid stellar tracers in resolved dwarf spheroidal galaxies. Empirical evidence indicates that all the dSphs that have been searched for evolved helium burning variable stars host RRLs. It is worth mentioning that they have been identified in all stellar systems hosting a stellar population older than $10 \mathrm{Gyr}$ and an intermediate horizontal branch (HB) morphology, i.e., systems in which the mass distribution along the HB covers the RRL instability strip (IS). The stellar systems with ages younger than 10 Gyr typically display Galactic thin disk kinematics (Blanco-Cuaresma et al. 2015). This means that we still lack solid empirical evidence of RR Lyrae belonging to old thin disk open clusters (Bono et al. 2011). On the other hand, ACs have been identified in stellar systems more metal-poor than about $[\mathrm{Fe} / \mathrm{H}]=-1.6$ dex. Theoretical predictions indicate that helium burning sequence of intermediate-mass stars more metal-rich than the above limit do not cross the so-called Cepheid IS (Castellani \& degl'Innocenti 1995; Stetson et al. 2014b).

It goes without saying that the comparison of pulsation and evolutionary properties of evolved helium burning-variables in nearby stellar systems allows us to constrain their early formation (Fiorentino et al. 2012, 2015a; Coppola et al. 2013; Stetson et al. 2014b). The Carina dSph is a very interesting laboratory for investigating the above issues. In a previous investigation we compared the period distribution of the central helium burning variable stars in Carina with similar distributions in nearby dSphs and in the Large Magellanic Cloud (LMC) and we found that the old stellar populations in these systems share similar properties (Coppola et al. 2013, hereafter, Paper VI). On the other hand, the period distribution and the Bailey diagram (luminosity amplitude versus period) of ACs show significant differences among the above stellar systems. This evidence suggested that the properties of intermediate-age stellar populations might be affected both by environmental effects and structural parameters.

In this investigation we move toward a complete census of helium-burning variable stars. The structure of the paper is the following. In Section 2 we present in detail the adopted photometric data set together with the light curves of both RRLs and ACs and their pulsation properties. In Section 3 we discuss the properties of RRLs and ACs using the Bailey diagram and the Petersen diagram (period ratio versus period). We focused our attention on the position of double mode variables and on the sensitivity of the period ratio on the iron abundance. In Section 4 we use the optical Period-WesenheitMetallicity (PWZ) relation of RRLs to estimate the Carina true distance modulus. To constrain the possible occurrence of systematic errors we estimated the Carina distance using the five field RRLs for which accurate estimates of their trigonometric parallaxes are available. Independent distance determinations were also provided using predicted optical PWZ relations. In Section 5 we present preliminary results about SX Phoenicis variables. Finally, in Section 6 we summarize the results of this investigation and briefly outline the future development of the Carina project.

\section{PHOTOMETRIC DATA}

The photometric catalog adopted in this investigation is an extension of the data set discussed by Coppola et al. (2013). This included $4474 \mathrm{CCD}$ images in the UBVRI photometric bands obtained with the CTIO $4 \mathrm{~m}$ and $1.5 \mathrm{~m}$ telescopes, the ESO $3.6 \mathrm{~m} \mathrm{NTT}$, the MPI/ESO $2.2 \mathrm{~m}$ telescope, and the $8 \mathrm{~m}$ ESO VLT during the period 1992 December through 2008 September. Here we add 2028 CCD images in the same photometric bands obtained with the same telescopes (see Table 1). In the current analysis, the $R$-band data were not included because the number of measurements is limited and they do not provide a good coverage of the pulsation cycle. The seeing had a median value of $1 . " 0$, and ranged from $0 . " 8$ (10percentile) and 0.9 (25-percentile) to 1 ."3 (75-percentile) and 1."6 (90-percentile). The reader interested in a detailed discussion of the photometric methodology, the absolute photometric calibration, the identification of the variable stars and analysis of the time series data is referred to Paper VI and to Stetson et al. (2014b).

The list of variable stars begins with that from Dall'Ora et al. (2003, hereafter D03), who merged the prior lists of Saha et al. (1986, hereafter S86), and performed their own comprehensive investigation of the resulting set of 92 candidate variable stars. This existing star list has been augmented by our own search for new variables within the new, expanded set of 6992 optical images of Carina. In particular, we have identified 92 RRLs. Among them 12 pulsate in the first-overtone $\left(\mathrm{FO}, \mathrm{RR}_{c}\right), 63$ in the fundamental mode $\left(\mathrm{F}, \mathrm{RR}_{a b}\right), 9$ are double-mode pulsators $\left(\mathrm{RR}_{d}\right)$ and 8 are candidate Blazhko variables (Bl). We also identified $20 \mathrm{ACs}, 2$ long-period variables (LPV) and 10 geometrical variables (eclipsing binaries, EB, and W Uma). The main difference between the current set of variable stars and those discussed in Paper VI is that we have identified 16 new variables. Among them are six RRLs, one AC, seven EBs 
Table 2

Catalog of Carina Variable Stars

\begin{tabular}{|c|c|c|c|c|c|c|c|}
\hline ID & $\alpha(\mathrm{J} 2000.0)$ & $\delta(\mathrm{J} 2000.0)$ & S86 & D03 & VM13 & Paper VI & Our \\
\hline$\ldots$ & & & $\ldots$ & $\ldots$ & & & $\ldots$ \\
\hline$\overline{\mathrm{V} 3}$ & 064331.71 & -505105.3 & $\mathrm{ab}$ & $\ldots$ & $\ldots$ & V215 (ab) & $a b$ \\
\hline V4 & 064328.70 & -505546.9 & $\mathrm{c}$ & V177 (c) & $\ldots$ & $\ldots$ & $\mathrm{c}$ \\
\hline V7 & $0643 \quad 16.26$ & $\begin{array}{llll}-51 & 04 & 17.2\end{array}$ & $a b$ & V7 (ab) & $\ldots$ & V7 (ab) & $a b$ \\
\hline V10 & $0643 \quad 10.66$ & -504347.8 & $a b$ & V10 (ab) & $\ldots$ & V10 (ab) & $a b$ \\
\hline V11 & 064308.20 & -505047.9 & $\mathrm{c}$ & V11 (d) & $\ldots$ & V11 (d) & $\mathrm{d}$ \\
\hline V14 & 064301.93 & $\begin{array}{lll}-51 & 01 & 26.3\end{array}$ & $a b$ & V14 (AC) & $\ldots$ & V14 (AC) & $\mathrm{AC}$ \\
\hline $\mathrm{V} 22$ & 064246.32 & -505736.9 & $a b$ & $\mathrm{~V} 22(\mathrm{c})$ & $\ldots$ & $\mathrm{V} 22(\mathrm{ab})$ & $a b$ \\
\hline V24 & 064234.00 & -504424.9 & $a b$ & V24 (ab) & $\ldots$ & V24 (ab) & $a b$ \\
\hline V25 & $0642 \quad 32.22$ & -505936.4 & $a b$ & $\ldots$ & $\ldots$ & $\mathrm{V} 213(\mathrm{ab})$ & $\mathrm{B} 1$ \\
\hline V26 & 064230.33 & -505224.1 & $\mathrm{ab}$ & V26 (d) & $\ldots$ & V26 (d) & $\mathrm{d}$ \\
\hline V27 & $0642 \quad 28.89$ & -504750.2 & $a b$ & V27 (AC) & $\ldots$ & $\mathrm{V} 27$ (AC) & $\mathrm{AC}$ \\
\hline V29 & $0642 \quad 25.07$ & -510347.7 & $a b$ & V29 (AC) & $\ldots$ & $\mathrm{V} 29(\mathrm{AC})$ & $\mathrm{AC}$ \\
\hline V30 & $0642 \quad 24.10$ & -510238.9 & $a b$ & $\mathrm{~V} 30(\mathrm{ab})$ & $\ldots$ & $\mathrm{V} 30(\mathrm{ab})$ & $a b$ \\
\hline V31 & $0642 \quad 22.87$ & -505916.0 & $\mathrm{c}$ & V31 (ab) & $\ldots$ & V31 (ab) & $\mathrm{B} 1$ \\
\hline V32 & 064222.50 & -505918.3 & $a b$ & V202 (ab) & $\ldots$ & V202 (ab) & $\mathrm{ab}$ \\
\hline V33 & 064221.10 & -511151.4 & $a b$ & V33 (AC) & $\ldots$ & V33 (AC) & $\mathrm{AC}$ \\
\hline V34 & $0642 \quad 19.22$ & -504530.7 & $a b$ & V34 (ab) & $\ldots$ & V34 (ab) & $\mathrm{ab}$ \\
\hline V40 & $0642 \quad 15.63$ & -510700.0 & $\mathrm{c}(\mathrm{ab} ?)$ & $\mathrm{V} 40(\mathrm{c})$ & $\ldots$ & V40 (c) & $\mathrm{c}$ \\
\hline V41 & $0642 \quad 14.76$ & -505514.0 & SV & V180 (AC) & $\ldots$ & $\mathrm{V} 180(\mathrm{AC})$ & $\mathrm{AC}$ \\
\hline V43 & $0642 \quad 13.11$ & -504649.9 & $\mathrm{c}$ & $\mathrm{V} 43(\mathrm{c})$ & $\ldots$ & V43 (c) & $\mathrm{c}$ \\
\hline V44 & $0642 \quad 13.05$ & -50 5722.1 & $a b$ & $\ldots$ & $\ldots$ & $\mathrm{V} 212(\mathrm{ab})$ & $a b$ \\
\hline V47 & 064209.10 & -505353.5 & $\mathrm{c}$ & V47 (c) & $37(a b)$ & V47 (c) & $\mathrm{c}$ \\
\hline V49 & 064208.77 & -510111.8 & $a b$ & V49 (ab) & $\ldots$ & V49 (ab) & $a b$ \\
\hline V57 & 064202.85 & -505256.8 & $a b$ & V57 (ab) & $35(a b)$ & V57 (ab) & $a b$ \\
\hline V58 & 064201.04 & -505719.7 & $a b$ & $\ldots$ & $34(\mathrm{ab})$ & V211 (ab) & $a b$ \\
\hline V60 & 064159.75 & -510638.5 & $a b$ & V60 (ab) & $33(a b)$ & V60 (ab) & $a b$ \\
\hline V61 & 064158.29 & -504737.8 & $a b$ & V61 (ab) & $32(a b)$ & V61 (ab) & $\mathrm{B} 1$ \\
\hline V65 & 064155.86 & -505535.3 & $a b$ & V65 (ab) & $31(\mathrm{ab})$ & V65 (ab) & $a b$ \\
\hline V67 & 064152.64 & -510519.7 & $a b$ & V67 (ab) & $30(a b)$ & V67 (ab) & $a b$ \\
\hline V68 & 064149.15 & -505918.7 & $a b$ & V68 (ab) & $28(a b)$ & V68 (ab) & $a b$ \\
\hline V73 & 064146.91 & -510707.5 & $a b$ & V73 (ab) & $27(\mathrm{ab})$ & V73 (ab) & $a b$ \\
\hline V74 & 064143.86 & -505409.0 & $\mathrm{c}$ & V74 (c) & $\ldots$ & V74 (c) & $\mathrm{d}$ \\
\hline V75 & 064142.90 & -505849.5 & $a b$ & $\ldots$ & $25(a b)$ & $\ldots$ & $a b$ \\
\hline V77 & 064139.24 & -510539.7 & $a b$ & V77 (ab) & $23(a b)$ & V77 (Bl) & $\mathrm{B} 1$ \\
\hline V84 & 064137.11 & -510004.5 & $a b$ & V84 (ab) & $\ldots$ & V84 (ab) & $a b$ \\
\hline V85 & 064135.64 & -505007.4 & $a b$ & V85 (ab) & $22(a b)$ & V85 (ab) & $\mathrm{ab}$ \\
\hline V87 & 064132.67 & -505700.8 & SV & V87 (AC) & $3(\mathrm{AC})$ & V87 (AC) & $\mathrm{AC}$ \\
\hline V89 & 064129.94 & -504713.7 & $a b$ & V89 (d) & $21(a b)$ & V89 (d) & $\mathrm{d}$ \\
\hline V90 & 064129.92 & -505210.7 & $a b$ & V90(ab) & $20(a b)$ & V90 (ab) & $\mathrm{ab}$ \\
\hline V91 & 064129.46 & -510428.5 & $a b$ & V91 (ab) & $19(\mathrm{ab})$ & V91 (ab) & $a b$ \\
\hline V92 & 064128.75 & -510650.5 & $a b$ & V92 (ab) & $18(a b)$ & V92 (ab) & $a b$ \\
\hline V105 & $0641 \quad 16.53$ & -510903.0 & $a b$ & V105 (ab) & $14(\mathrm{ab})$ & V105 (ab) & $\mathrm{ab}$ \\
\hline V115 & 064106.23 & -505318.5 & $?$ & V115 (AC) & $\ldots$ & V115 (AC) & $\mathrm{AC}$ \\
\hline V116 & 064105.63 & -510028.1 & $a b$ & V116 (ab) & $11(\mathrm{ab})$ & V116 (ab) & $\mathrm{ab}$ \\
\hline V122 & 064056.97 & -510045.8 & $a b$ & V122(ab) & $\ldots$ & V122 (ab) & $a b$ \\
\hline V123 & 064054.92 & -504414.5 & $a b$ & V123 (ab) & $\ldots$ & V123 (ab) & $a b$ \\
\hline V124 & 064053.78 & -510553.1 & $a b$ & V124 (ab) & $\ldots$ & V124 (ab) & $a b$ \\
\hline V125 & 064053.37 & -505853.3 & $a b$ & V125 (ab) & 10 (c) & V125 (ab) & $a b$ \\
\hline V126 & 064052.35 & -505856.6 & $a b$ & V126 (ab) & $\ldots$ & V126 (Bl) & $\mathrm{B} 1$ \\
\hline V127 & 064052.22 & -505920.8 & $a b$ & V127 (ab) & $\ldots$ & V127 (B1) & $\mathrm{B} 1$ \\
\hline V129 & 064049.34 & -510815.0 & $a b$ & V129 (AC) & $\ldots$ & V129 (AC) & $\mathrm{AC}$ \\
\hline V133 & 064047.93 & $\begin{array}{lll}-51 & 03 & 23.5\end{array}$ & $a b$ & $\ldots$ & $\ldots$ & V209 (ab) & $\mathrm{ab}$ \\
\hline V135 & 064046.43 & -510524.6 & $a b$ & V135 (ab) & $\ldots$ & V135 (ab) & $a b$ \\
\hline V136 & 064044.80 & $\begin{array}{lll}-51 & 12 & 42.7\end{array}$ & $a b$ & V136 (ab) & $\ldots$ & V136 (ab) & $a b$ \\
\hline V138 & 064040.73 & -505258.3 & SV & V138 (ab) & $\ldots$ & V138 (ab) & $a b$ \\
\hline V141 & 064039.29 & -505601.3 & SV & V141 (ab) & $\begin{array}{l}\cdots \\
\cdots\end{array}$ & V141 (ab) & $\mathrm{ab}$ \\
\hline V142 & 064038.40 & -510138.8 & SV & V142 (c) & $\ldots$ & $\mathrm{V} 142(\mathrm{c})$ & $\mathrm{c}$ \\
\hline V143 & 064038.06 & -511130.9 & $\mathrm{ab}$ & V143 (ab) & $\ldots$ & V143 (ab) & $a b$ \\
\hline V144 & 064037.20 & -505925.0 & $\mathrm{c}$ & V144 (c) & $9(\mathrm{c})$ & V144 (c) & $\mathrm{c}$ \\
\hline V148 & 064031.69 & -504839.9 & $a b$ & V148(c) & 8 (c) & V148 (c) & $\mathrm{c}$ \\
\hline V149 & 064030.46 & -510700.9 & $a b$ & V149 (AC) & $\ldots$ & V149 (AC) & $\mathrm{AC}$ \\
\hline
\end{tabular}


Table 2

(Continued)

\begin{tabular}{|c|c|c|c|c|c|c|c|}
\hline $\begin{array}{l}\text { ID } \\
\ldots\end{array}$ & $\begin{array}{l}\alpha(\mathrm{J} 2000.0) \\
(\mathrm{hh} \mathrm{mm} \mathrm{ss})\end{array}$ & $\begin{array}{l}\delta(\mathrm{J} 2000.0) \\
(\mathrm{dd} \mathrm{mm} \mathrm{ss})\end{array}$ & $\begin{array}{c}\text { S86 } \\
\ldots\end{array}$ & $\begin{array}{l}\text { D03 } \\
\ldots\end{array}$ & $\begin{array}{l}\text { VM13 } \\
\ldots\end{array}$ & $\begin{array}{l}\text { Paper VI } \\
\ldots\end{array}$ & $\begin{array}{c}\text { Our } \\
\ldots\end{array}$ \\
\hline V151 & 064029.45 & -510017.3 & SV & V151 (c) & 7 (c) & V151 (c) & $\mathrm{c}$ \\
\hline V153 & 064024.39 & -510449.9 & $a b$ & V153 (ab) & $\ldots$ & V153 (ab) & $a b$ \\
\hline V158 & 064012.87 & -505624.8 & $a b$ & V158 (ab) & $\ldots$ & V158 (ab) & $a b$ \\
\hline V159 & 064012.59 & $\begin{array}{lll}-51 & 09 & 35.8\end{array}$ & $a b$ & V159 (ab) & $\ldots$ & V159 (ab) & $a b$ \\
\hline V164 & 063956.29 & -505354.7 & $a b$ & V164 (ab) & $\ldots$ & V164 (ab) & $a b$ \\
\hline V165 & 063955.72 & -505949.2 & $a b$ & V186 (ab) & $\ldots$ & V186 (ab) & $\mathrm{ab}$ \\
\hline V170 & 063938.54 & -504633.6 & $a b$ & $\ldots$ & $\ldots$ & $\ldots$ & $a b$ \\
\hline V171 & 063936.54 & $\begin{array}{lll}-51 & 1240.7\end{array}$ & $a b$ & $\ldots$ & $\ldots$ & $\ldots$ & $\mathrm{ab}$ \\
\hline V173 & 064327.13 & -504449.2 & $\ldots$ & V173 (RGB) & $\ldots$ & V173 (RGB) & RGB \\
\hline V174 & 064238.13 & -504610.9 & $\ldots$ & V174 (ab) & $\ldots$ & V174 (ab) & $\mathrm{ab}$ \\
\hline V175 & 064254.36 & -505121.6 & $\ldots$ & V175 (c) & $\ldots$ & V175 (c) & $\mathrm{c}$ \\
\hline V176 & 064300.06 & -505359.1 & $\ldots$ & V176 (c) & $\ldots$ & V176 (ab) & $a b$ \\
\hline V178 & 064144.04 & -505017.2 & $\ldots$ & V178 (AC) & $5(\mathrm{AC})$ & V178 (AC) & $\mathrm{AC}$ \\
\hline V179 & 064149.40 & -505411.2 & $\ldots$ & V179 (ab) & 29 (c) & V179 (ab) & $\mathrm{ab}$ \\
\hline V181 & 064224.16 & -505600.3 & $\ldots$ & V181 (c) & $\ldots$ & V181 (c) & $\mathrm{c}$ \\
\hline V182 & 064134.93 & -505239.1 & $\ldots$ & V182 (ab) & $\ldots$ & V182 (ab) & $\mathrm{ab}$ \\
\hline V183 & 064127.59 & -505601.4 & $\ldots$ & V183 (ab) & $17(a b)$ & V183 (ab) & $a b$ \\
\hline V184 & 064033.02 & -504314.8 & $\ldots$ & V184 (c) & $\ldots$ & V184 (c) & $\mathrm{c}$ \\
\hline V185 & 064025.22 & -504435.4 & $\ldots$ & $\mathrm{V} 185(\mathrm{ab})$ & $\ldots$ & V185 (ab) & $\mathrm{ab}$ \\
\hline V187 & 064024.74 & $\begin{array}{lll}-51 & 01 & 29.4\end{array}$ & $\ldots$ & V187 (AC) & $\ldots$ & V187 (AC) & $\mathrm{AC}$ \\
\hline V188 & $\begin{array}{lll}06 & 40.28\end{array}$ & -510706.2 & $\ldots$ & V188 (ab) & $\ldots$ & V188 (ab) & $a b$ \\
\hline V189 & 064009.85 & -510805.2 & $\ldots$ & V189 (ab) & $6(\mathrm{c})$ & V189 (ab) & $a b$ \\
\hline V190 & 064139.78 & -505902.1 & $\ldots$ & V190 (AC) & $4(\mathrm{AC})$ & V190 (AC) & $\mathrm{AC}$ \\
\hline V191 & 064126.22 & -505933.9 & $\ldots$ & V191 (ab) & $16(a b)$ & V191 (ab) & $a b$ \\
\hline V192 & 064120.66 & -510031.5 & $\ldots$ & V192 (d) & $15(a b)$ & V192 (d) & $\mathrm{d}$ \\
\hline V193 & 064128.23 & -510045.4 & $\ldots$ & V193 (AC) & $2(\mathrm{AC})$ & V193 (AC) & $\mathrm{AC}$ \\
\hline V194 & 064104.23 & $\begin{array}{lll}-51 & 0132.9\end{array}$ & $\ldots$ & V194 (FV) & $\ldots$ & V194 (FV) & FV \\
\hline V195 & 064140.82 & $\begin{array}{lll}-51 & 0133.7\end{array}$ & $\ldots$ & V195 (ab) & $24(a b)$ & V195 (ab) & $a b$ \\
\hline V196 & 064111.48 & -510216.1 & $\ldots$ & V196 (ab) & $13(a b)$ & V196 (ab) & $a b$ \\
\hline V197 & 064100.27 & -510239.5 & $\ldots$ & V197 (c) & $\ldots$ & V197 (c) & $\mathrm{c}$ \\
\hline V198 & $0641 \quad 10.55$ & $\begin{array}{lll}-51 & 03 & 22.9\end{array}$ & $\ldots$ & V198 (d) & 12 (c) & V198 (d) & $\mathrm{d}$ \\
\hline V199 & $0641 \quad 19.60$ & $\begin{array}{lll}-51 & 10 & 23.7\end{array}$ & $\ldots$ & V199 (ab) & $\ldots$ & V199 (ab) & $\mathrm{ab}$ \\
\hline V200 & 064146.74 & -505839.3 & $\ldots$ & V200 (ab) & $26(a b)$ & V200 (ab) & $a b$ \\
\hline V201 & $\begin{array}{lll}06 & 42 & 10.38\end{array}$ & -505907.5 & $\ldots$ & V201 (ab) & $\ldots$ & V201 (ab) & $a b$ \\
\hline V203 & 064156.12 & -505921.6 & $\ldots$ & V203 (AC) & $6(\mathrm{AC})$ & V203 (AC) & $\mathrm{AC}$ \\
\hline V204 & 064204.10 & -510151.4 & $\ldots$ & V204 (ab) & $\ldots$ & V204 (ab) & $\mathrm{ab}$ \\
\hline V205 & $0642 \quad 24.51$ & $\begin{array}{lll}-51 & 02 & 40.4\end{array}$ & $\ldots$ & V205 (AC) & $7(\mathrm{AC})$ & V205 (AC) & $\mathrm{AC}$ \\
\hline V206 & 064204.25 & -510941.3 & $\ldots$ & V206 (ab) & $36(a b)$ & V206 (ab) & $\mathrm{B} 1$ \\
\hline V207 & 064307.50 & -510230.9 & $\ldots$ & V207 (d) & $\ldots$ & V207 (d) & $\mathrm{d}$ \\
\hline V208* & 064022.40 & $\begin{array}{lll}-51 & 17 & 08.1\end{array}$ & $\begin{array}{l}\cdots \\
\ldots\end{array}$ & $\ldots$ & $\cdots$ & V208 (ab) & $\mathrm{ab}$ \\
\hline V210* & 064127.04 & $\begin{array}{lll}-51 & 1539.8\end{array}$ & $\ldots$ & $\ldots$ & $\ldots$ & $\mathrm{V} 210(\mathrm{Bl})$ & $\mathrm{d}$ \\
\hline V214* & 064236.99 & $\begin{array}{lll}-51 & 03 & 29.7\end{array}$ & $\ldots$ & $\ldots$ & $\ldots$ & V214 (B1) & $\mathrm{B} 1$ \\
\hline V216* & $0641 \quad 17.76$ & -505646.0 & $\ldots$ & $\ldots$ & $\ldots$ & $\mathrm{V} 216$ (AC) & $\mathrm{AC}$ \\
\hline V217* & 064133.03 & -504756.0 & $\ldots$ & $\ldots$ & $\ldots$ & V217 (AC) & $\mathrm{AC}$ \\
\hline V218* & 064139.56 & -511552.6 & $\ldots$ & $\ldots$ & $\ldots$ & V218 (AC) & $\mathrm{AC}$ \\
\hline V219* & 064209.01 & -505937.6 & $\ldots$ & $\ldots$ & $\ldots$ & V219 (AC) & $\mathrm{AC}$ \\
\hline V220* & 064019.12 & -505823.0 & $\ldots$ & $\ldots$ & $\ldots$ & V220 (EB) & EB \\
\hline $\mathrm{V} 221^{*}$ & 064320.73 & -505529.9 & $\ldots$ & $\ldots$ & $\ldots$ & V221 (WUma) & WUma \\
\hline V222* & 064124.30 & $\begin{array}{lll}-51 & 0324.7\end{array}$ & $\ldots$ & $\ldots$ & $\ldots$ & $\ldots$ & LPV \\
\hline V223* & 063952.92 & -505428.3 & $\ldots$ & $\ldots$ & $\ldots$ & $\ldots$ & $a b$ \\
\hline V224* & 064036.37 & $\begin{array}{lll}-51 & 16 & 42.2\end{array}$ & $\cdots$ & $\cdots$ & $\cdots$ & $\cdots$ & LPV \\
\hline V225* & 064101.01 & $\begin{array}{lll}-51 & 00 & 02.4\end{array}$ & $\begin{array}{l}\cdots \\
\cdots\end{array}$ & $\cdots$ & $\cdots$ & $\begin{array}{l}\cdots \\
\ldots\end{array}$ & $\mathrm{d}$ \\
\hline V226* & 064018.76 & -511836.6 & $\ldots$ & $\ldots$ & $\ldots$ & $\ldots$ & $\mathrm{ab}$ \\
\hline V $227^{*}$ & 064126.13 & $\begin{array}{lll}-51 & 21 & 26.7\end{array}$ & $\ldots$ & $\cdots$ & $\cdots$ & $\ldots$ & $a b$ \\
\hline V228* & 064235.07 & -503807.2 & $\begin{array}{l}\cdots \\
\ldots\end{array}$ & $\cdots$ & $\begin{array}{l}\cdots \\
\ldots\end{array}$ & $\begin{array}{l}\cdots \\
\ldots\end{array}$ & $a b$ \\
\hline V229* & 063938.92 & -510559.1 & $\begin{array}{l}\cdots \\
\cdots\end{array}$ & $\begin{array}{l}\cdots \\
\cdots\end{array}$ & $\begin{array}{l}\cdots \\
\cdots\end{array}$ & $\begin{array}{l}\cdots \\
\ldots\end{array}$ & $a b$ \\
\hline V230* & 064234.11 & -505820.0 & $\ldots$ & $\ldots$ & $8(\mathrm{AC})$ & $\ldots$ & $\mathrm{AC}$ \\
\hline V231* & $0641 \quad 10.58$ & -505340.1 & $\ldots$ & $\ldots$ & $\ldots$ & $\ldots$ & $\mathrm{EB}$ \\
\hline V232* & 064010.11 & -511601.5 & $\ldots$ & $\ldots$ & $\ldots$ & $\ldots$ & EB \\
\hline V233* & 064123.47 & -505403.0 & $\ldots$ & $\ldots$ & $\ldots$ & $\ldots$ & EB \\
\hline V234* & 064230.62 & -505334.5 & $\ldots$ & $\ldots$ & $\ldots$ & $\ldots$ & EB \\
\hline
\end{tabular}


Table 2

(Continued)

\begin{tabular}{|c|c|c|c|c|c|c|c|}
\hline $\begin{array}{l}\text { ID } \\
\ldots\end{array}$ & $\begin{array}{l}\alpha(\mathrm{J} 2000.0) \\
(\mathrm{hh} \mathrm{mm} \mathrm{ss})\end{array}$ & $\begin{array}{l}\delta(\mathrm{J} 2000.0) \\
(\mathrm{dd} \mathrm{mm} \mathrm{ss})\end{array}$ & $\begin{array}{c}\text { S86 } \\
\ldots\end{array}$ & $\begin{array}{l}\text { D03 } \\
\ldots\end{array}$ & $\begin{array}{l}\text { VM13 } \\
\ldots\end{array}$ & $\begin{array}{l}\text { Paper VI } \\
\ldots\end{array}$ & $\begin{array}{c}\text { Our } \\
\ldots\end{array}$ \\
\hline V235* & $0643 \quad 14.32$ & -510645.0 & $\ldots$ & $\ldots$ & $\ldots$ & $\ldots$ & EB \\
\hline V236* & 064103.78 & -515452.8 & $\ldots$ & $\ldots$ & 2 (Mis) & $\ldots$ & EB \\
\hline
\end{tabular}

Notes. Col. 1: Identification star; Col. 2: R.A., $(\alpha)$; Col. 3: Decl., ( $\delta$; Col. 4: Type as in S86; Col. 5: Type and identification as in D03; Col. 6: Type and identification as in VM13; Col. 7: Identification and type as in Paper VI; Col. 8: Type as in this study. FV—Field variable. RGB_Variable located along the RGB. SV—Suspected variable.

(This table is available in machine-readable form.)

and two probable LPVs. Moreover, in Paper VI we discovered 14 new variables, but by using the new data set, we confirm that only nine of these are new identifications (see the Appendix for details).

Recently Vivas \& Mateo (2013, hereafter VM13) performed a detailed investigation of pulsating stars in Carina. They found 38 RRLs, 10 AC variables and more than 340 new SX Phoenicis (SX Phe). Among them 36 (30 RRLs and 6 ACs) are in common with D03 and 41 (32 RRLs, 7 ACs, 2 EBs) with our new catalog. The nine stars listed by VM13 that do not appear in our catalog are listed in Table 7 . These variables were not included in the following analysis of RRLs pulsation properties since they are located beyond the Carina tidal radius (see Section 3.1 for a detailed discussion). Concerning SX Phe stars, we confirm the variability for 324 out of 340 known pulsators in Carina and we identified 101 new variables of this class (see Section 5 for details).

In the first column of Table 2 we find the identification number according to S86 and D03. For the newly detected variables in this paper and in Paper VI (208-237) the D03 running number was continued and these stars were also marked with an asterisk in this same table. Columns (2) and (3) list $\alpha$ and $\delta$ (J2000.0) coordinates in units of (hh:mm:ss) and (dd:mm:ss), respectively, ${ }^{12}$ while the last five columns give S86, D03, VM13, Paper VI and current individual notes. Candidate variable stars for which we could not confirm the variability are given in Table 3 .

The current data set included the $B V$ images adopted by D03 in their investigation of Carina's variable stars. We confirm their variability analysis and their variable star classification with only a few exceptions. According to our extended data set the variable V161 does not show clear signs of variability. The variable V17 appears to be an EB variable; while the variables V22 and V176 seem to pulsate in the fundamental $\left(\mathrm{RR}_{a b}\right)$ instead of in the first overtone $\left(\mathrm{RR}_{c}\right)$ mode. The variables V31, V61, V77, V126, V127 and V206 were also classified by D03 as $\mathrm{RR}_{a b}$ variables and according to our new data they also show the Blazhko effect. Moreover, the variable V74 was classified by $\mathrm{D} 03$ as $\mathrm{RR}_{c}$ variable, but according to our new data it is classified as a double-mode pulsator. A more detailed analysis of individual variables is given in the Appendix.

The individual $U B V I$ measurements for all the variables in our sample are listed in Table 4. For every variable in our sample the first three columns of the table contain the Heliocentric Julian Date (HJD), the $U$-band magnitude and the photometric error. Columns (4)-(12) give the same

\footnotetext{
${ }^{12}$ The astrometry is on the system of the USNO-A2.0 catalog.
}

information, but for the $B-, V$-, and $I$-band measurements. The total number of phase points in the complete data set depends on the photometric band, and they range from 1 to 23 $(U)$, from 22 to $203(B)$, from 38 to $278(V)$ and from 3 to 92 (I). The coverage of the pulsation cycle is optimal in the $B$ and $V$ bands, modest in the $I$ band and poor in the $U$ band. The photometric error of individual measurements depends on the photometric band and on seeing conditions. It is also occasionally affected by crowding conditions, but it is on average of the order of $0.02 \mathrm{mag}$.

Period searches and the phasing of light curves were performed using the procedure adopted by Stetson et al. (2014a), for the galactic Globular Cluster M4. The large time baseline ( $\sim 20$ years) covered by our data set allowed us to overcome half- and one-day alias ambiguities that can be quite severe in data sets covering limited time intervals. The large time interval covered by the current data set allowed us to provide more precise periods, epochs of maximum light, and luminosity amplitudes for the entire set of variable stars. In particular, the accuracy of the period estimates is related to the period itself and to the time interval covered by the observations. They range from $\left(1 \times 10^{-8}\right)$ to $\left(1 \times 10^{-5}\right)$ days.

The mean optical magnitudes and the amplitudes in the bands with good time sampling $(B, V)$ were estimated from fits with a spline under tension.

We already mentioned that the time sampling of both the $U$ and $I$-band light curves is either modest or poor. This means less accurate mean magnitudes and luminosity amplitudes. To overcome this problem for the $I$-band light curves we adopted the method described by Di Criscienzo et al. (2011) based on using the $V$-band light curve as a template and re-scaling the same measurements in amplitude to fit the I-band light curves. To accomplish this goal we adopted a visual-to-I-band amplitude ratio of $1.58 \pm 0.03$, obtained by $\mathrm{Di}$ Criscienzo et al. (2011) using the literature $V$ and $I$ light curves of 130 RRLs with good light curve parameters selected from different Galactic globular clusters.

The $U$-band light curves for several variables are poorly sampled, and often there is no coverage across minimum and/or maximum light. In those cases, we adopted as a mean magnitude the median of measurements and we do not provide luminosity amplitudes.

Table 5 lists, from left to right, for every variable in the current data set, the identification, the classification, the epoch of maximum light, the period (days), the intensity-averaged [ $\langle U\rangle,\langle B\rangle,\langle V\rangle,\langle I\rangle]$ mean magnitudes and the $A(U), A(B), A(V), A$ (I) luminosity amplitudes. For some variables the light curves are poorly sampled, and as explained above for the $U$ band, we 
Table 3

Catalog of Candidate Variable Stars that According to the Current Investigation are Not Variable

\begin{tabular}{|c|c|c|c|c|c|}
\hline $\begin{array}{l}\text { ID } \\
\ldots \\
\end{array}$ & $\begin{array}{l}\alpha(\mathrm{J} 2000.0) \\
(\mathrm{hh} \mathrm{mm} \mathrm{ss})\end{array}$ & $\begin{array}{l}\delta(\mathrm{J} 2000.0) \\
(\mathrm{dd} \mathrm{mm} \mathrm{ss})\end{array}$ & $\begin{array}{l}\text { S86 } \\
\ldots \\
\end{array}$ & $\begin{array}{l}\text { D03 } \\
\ldots \\
\end{array}$ & $\begin{array}{l}\text { This Work } \\
\ldots\end{array}$ \\
\hline V1 & 064221.90 & -505135.0 & $a b$ & $\ldots$ & NV \\
\hline V2 & 064219.60 & -504512.0 & SV & $\ldots$ & NV \\
\hline V5 & 064319.04 & -505329.3 & SV & $\mathrm{NV}$ & NV \\
\hline V6 & 064318.01 & -504643.7 & SV & $\mathrm{NV}$ & NV \\
\hline V8 & 064256.44 & -504145.6 & $\delta \mathrm{Scu}$ & NV & NV \\
\hline V9 & $0643 \quad 16.00$ & -510243.6 & WUma & NV & $\mathrm{NV}^{\mathrm{a}}$ \\
\hline V12 & 064306.20 & -505904.6 & $\delta \mathrm{Scu}$ & NV & $\mathrm{NV}^{\mathrm{b}}$ \\
\hline V13 & 064305.02 & -504933.0 & SV & NV & $N V^{b}$ \\
\hline V15 & 064300.82 & -505028.2 & SV & NV & $\mathrm{NV}$ \\
\hline V16 & 064256.33 & $-5054 \quad 47.4$ & SV & NV & $\mathrm{NV}^{\mathrm{b}}$ \\
\hline V17 & 064256.39 & -510838.0 & SV & NV & $\mathrm{EB}$ \\
\hline V18 & 063730.80 & -511731.4 & SV & $\mathrm{NV}^{\mathrm{c}}$ & NV \\
\hline V19 & 064254.24 & -504444.8 & SV & NV & NV \\
\hline V20 & 064250.97 & -505606.7 & SV & $\mathrm{NV}$ & NV \\
\hline V21 & 064246.66 & -510850.6 & SV & $\mathrm{NV}$ & NV \\
\hline V23 & 064237.03 & -510328.6 & $\mathrm{ab}$ & $\ldots$ & NV \\
\hline V28 & 064226.26 & -505944.2 & SV & $\mathrm{NV}^{\mathrm{c}}$ & $\mathrm{NV}^{\mathrm{a}}$ \\
\hline V35 & 064219.37 & -504844.0 & SV & NV & $\mathrm{NV}$ \\
\hline V36 & $0642 \quad 18.15$ & -510807.6 & SV & $\mathrm{NV}$ & $\mathrm{NV}$ \\
\hline V37 & 064152.94 & -504411.4 & SV & NV & $\mathrm{NV}^{\mathrm{d}}$ \\
\hline V38 & $0642 \quad 17.30$ & -510716.0 & SV & $\mathrm{NV}$ & $N V^{b}$ \\
\hline V39 & $06 \quad 42 \quad 17.21$ & -510158.0 & SV & $\mathrm{NV}^{\mathrm{c}}$ & NV \\
\hline $\mathrm{V} 42$ & $0642 \quad 14.75$ & -505536.0 & SV & NV & $\mathrm{NV}^{\mathrm{b}}$ \\
\hline V45 & $0642 \quad 10.98$ & -511256.5 & SV & $\mathrm{NV}$ & $\mathrm{NV}$ \\
\hline V46 & 064208.71 & -504657.3 & SV & NV & $N V^{b}$ \\
\hline V48 & 064208.98 & $\begin{array}{lll}-51 & 02 & 40.4\end{array}$ & SV & $\mathrm{NV}$ & $\mathrm{NV}$ \\
\hline V50 & 064208.50 & -510252.1 & SV & $\mathrm{NV}$ & NV \\
\hline V51 & 064208.32 & $\begin{array}{lll}-51 & 02 & 26.7\end{array}$ & SV & $\mathrm{NV}$ & $\mathrm{NV}$ \\
\hline V52 & $0642 \quad 07.52$ & -504308.4 & SV & NV & NV \\
\hline V53 & 064207.46 & -505125.3 & SV & NV & NV \\
\hline V54 & 064207.54 & $\begin{array}{lll}-51 & 01 & 17.0\end{array}$ & SV & $\mathrm{NV}$ & NV \\
\hline V55 & 064231.74 & -505156.7 & SV & $\mathrm{NV}$ & $\mathrm{NV}^{\mathrm{e}}$ \\
\hline V56 & 064204.38 & -504915.1 & SV & $\mathrm{NV}$ & $\mathrm{NV}$ \\
\hline V59 & 064159.71 & -505037.3 & SV & NV & NV \\
\hline V62 & 064157.81 & -505953.3 & SV & NV & $\mathrm{NV}^{\mathrm{b}}$ \\
\hline V63 & 064157.40 & -510323.0 & SV & $\mathrm{NV}$ & $\mathrm{NV}$ \\
\hline V64 & 064157.17 & -505330.0 & SV & NV & $\mathrm{NV}^{\mathrm{b}}$ \\
\hline V66 & 064153.04 & $-51 \quad 1238.3$ & SV & NV & $\mathrm{NV}$ \\
\hline V69 & 064148.94 & -510232.5 & $\mathrm{c}$ & NV & $\mathrm{NV}^{\mathrm{a}}$ \\
\hline V70 & 064148.23 & -505501.8 & SV & $\mathrm{NV}$ & $\mathrm{NV}$ \\
\hline V71 & 064147.90 & -510312.6 & SV & $\mathrm{NV}$ & $\mathrm{NV}$ \\
\hline V72 & 064146.89 & -510525.3 & SV & NV & $\mathrm{NV}$ \\
\hline V76 & 064139.68 & -504856.1 & SV & NV & $\mathrm{NV}^{\mathrm{e}}$ \\
\hline V78 & 064026.70 & -510823.0 & SV & $\ldots$ & $\mathrm{NV}$ \\
\hline V79 & 064138.26 & -504903.5 & SV & $\mathrm{NV}$ & $\mathrm{NV}$ \\
\hline V80 & 064138.36 & -510437.5 & SV & NV & $\mathrm{NV}^{\mathrm{b}}$ \\
\hline V81 & 064137.70 & $\begin{array}{lll}-51 & 02 & 37.2\end{array}$ & SV & NV & NV \\
\hline V82 & 064137.52 & -505741.5 & SV & $\mathrm{NV}$ & $\mathrm{NV}^{\mathrm{c}}$ \\
\hline V83 & 064137.46 & -510137.1 & SV & NV & $\mathrm{NV}^{\mathrm{c}}$ \\
\hline V86 & 064133.86 & -504349.3 & SV & NV & NV \\
\hline V88 & 064131.18 & -505756.1 & SV & $\mathrm{NV}$ & $\mathrm{NV}$ \\
\hline V93 & $\begin{array}{llll}06 & 16.20\end{array}$ & $-51 \quad 1020.0$ & SV & $\ldots$ & $\mathrm{NV}$ \\
\hline V94 & 064014.60 & -505720.0 & SV & $\ldots$ & $\mathrm{NV}$ \\
\hline V95 & 064126.76 & -505215.0 & SV & NV & $\mathrm{NV}^{\mathrm{b}}$ \\
\hline V96 & 064124.98 & -504411.9 & SV & $\mathrm{NV}$ & $\mathrm{NV}$ \\
\hline V97 & 064009.40 & -505431.0 & SV & $\ldots$ & NV \\
\hline V98 & 064008.50 & -505717.0 & SV & $\ldots$ & $\mathrm{NV}$ \\
\hline V99 & $0641 \quad 18.87$ & -505824.8 & SV & $\mathrm{NV}$ & NV \\
\hline V100 & 064005.6 & -504348.0 & $\mathrm{c}$ & $\ldots$ & NV \\
\hline V101 & $0641 \quad 18.13$ & -510128.0 & SV & $\mathrm{NV}$ & $\mathrm{NV}$ \\
\hline V102 & $0641 \quad 18.12$ & $-51 \quad 1057.1$ & SV & NV & NV \\
\hline V103 & $0641 \quad 17.40$ & -505515.3 & SV & NV & NV \\
\hline V104 & $0641 \quad 16.45$ & -510108.8 & SV & NV & $N V^{b}$ \\
\hline
\end{tabular}

Table 3

(Continued)

\begin{tabular}{|c|c|c|c|c|c|}
\hline $\begin{array}{l}\text { ID } \\
\ldots\end{array}$ & $\begin{array}{l}\alpha \text { (J2000.0) } \\
(\text { hh mm ss) }\end{array}$ & $\begin{array}{l}\delta(\mathrm{J} 2000.0) \\
(\mathrm{dd} \mathrm{mm} \mathrm{ss})\end{array}$ & $\begin{array}{l}\text { S86 } \\
\ldots\end{array}$ & $\begin{array}{l}\text { D03 } \\
\ldots\end{array}$ & $\begin{array}{l}\text { This Work } \\
\ldots\end{array}$ \\
\hline V106 & $0641 \quad 15.10$ & $\begin{array}{lll}-51 & 08 & 40.7\end{array}$ & SV & NV & $\mathrm{NV}^{\mathrm{b}}$ \\
\hline V107 & $0641 \quad 11.36$ & $\begin{array}{llll}-51 & 11 & 14.8\end{array}$ & SV & NV & NV \\
\hline V108 & $0641 \quad 10.55$ & $\begin{array}{lll}-51 & 08 & 19.5\end{array}$ & SV & NV & NV \\
\hline V109 & 064109.53 & -505943.8 & SV & $\mathrm{NV}^{\mathrm{a}}$ & $N V^{a}$ \\
\hline V110 & 064109.18 & -510033.7 & SV & $\mathrm{NV}$ & $N V^{b}$ \\
\hline V111 & 063956.10 & -505436.0 & SV & $\ldots$ & NV \\
\hline V112 & 064108.80 & $\begin{array}{lll}-51 & 04 & 39.3\end{array}$ & SV & $\mathrm{NV}$ & NV \\
\hline V113 & 064108.56 & -504821.1 & SV & NV & NV \\
\hline V114 & 064108.26 & -505441.1 & SV & $\mathrm{NV}$ & $N V^{b}$ \\
\hline V117 & 063952.60 & -510007 & SV & $\ldots$ & NV \\
\hline V118 & 064101.82 & -504722.4 & SV & $\mathrm{NV}$ & $N V^{e}$ \\
\hline V119 & 064101.44 & -505230.7 & SV & NV & NV \\
\hline V120 & 064058.83 & -504524.4 & SV & NV & NV \\
\hline V121 & 064057.49 & -510041.2 & SV & $\mathrm{NV}$ & NV \\
\hline V128 & 064049.17 & $\begin{array}{lll}-51 & 00 & 33.4\end{array}$ & SV & $\ldots$ & $N V^{b}$ \\
\hline V130 & 063935.90 & -504313.0 & SV & $\ldots$ & NV \\
\hline V131 & 063936.50 & -510143.0 & SV & $\ldots$ & NV \\
\hline V132 & 063935.90 & -505438.0 & SV & $\ldots$ & NV \\
\hline V134 & 063935.80 & -510654.0 & SV & $\ldots$ & NV \\
\hline V137 & 064043.01 & -505554.3 & SV & $\mathrm{NV}$ & NV \\
\hline V139 & 064039.86 & $\begin{array}{lll}-50 & 5322.9\end{array}$ & SV & NV & NV \\
\hline V140 & 064039.47 & -510702.2 & SV & $\mathrm{NV}$ & $\mathrm{NV}$ \\
\hline V145 & 064036.06 & -505831.1 & SV & NV & NV \\
\hline V146 & 064034.79 & $\begin{array}{lll}-51 & 01 & 51.7\end{array}$ & SV & NV & NV \\
\hline V147 & 064034.08 & $\begin{array}{llll}-51 & 11 & 29.9\end{array}$ & SV & NV & $N V^{b}$ \\
\hline V150 & 064030.73 & $\begin{array}{l}-504527.9 \\
\end{array}$ & SV & NV & NV \\
\hline V152 & 064029.32 & -504214.5 & SV & $\mathrm{NV}^{\mathrm{c}}$ & $N V^{b}$ \\
\hline V154 & 064013.95 & -504255.1 & SV & NV & NV \\
\hline V155 & $\begin{array}{lll}06 & 19.68\end{array}$ & -504545.4 & SV & NV & NV \\
\hline V156 & $\begin{array}{llll}06 & 17.98\end{array}$ & -510602.0 & SV & NV & NV \\
\hline V157 & $\begin{array}{lll}06 & 17.60\end{array}$ & -505841.4 & SV & $\mathrm{NV}$ & NV \\
\hline V160 & 064001.42 & -510250.3 & SV & NV & NV \\
\hline V161 & 064000.98 & -510406.6 & SV & SV & $N V^{a}$ \\
\hline V162 & $0640 \quad 00.14$ & $\begin{array}{l}-504414.5 \\
\end{array}$ & SV & NV & NV \\
\hline V163 & 063957.08 & $\begin{array}{llll}-50 & 51 & 10.6\end{array}$ & SV & NV & NV \\
\hline V166 & 063829.90 & -505241.0 & SV & $\ldots$ & NV \\
\hline V167 & 063830.30 & -505436.0 & SV & $\ldots$ & NV \\
\hline V168 & 063829.90 & -505427.0 & SV & $\ldots$ & NV \\
\hline V169 & 063829.2 & -504505.0 & SV & $\ldots$ & NV \\
\hline V172 & 064108.38 & -504706.8 & SV & NV & NV \\
\hline
\end{tabular}

Notes. Col. 1: Identification star; Col. 2: R.A., $(\alpha)$; Col. 3: Decl., ( $\delta)$; Col. 4: Type as in S86; Col. 5: Type as in D03; Col. 6: Type as in this study. NV—No variability detected. SV-Suspected variable.

${ }^{\text {a }}$ Possible blend.

${ }^{\mathrm{b}}$ Located close to a bright or faint star.

${ }^{\mathrm{c}}$ Possible field galaxy.

${ }^{\mathrm{d}}$ Saturated star.

${ }^{\mathrm{e}}$ Faint star.

(This table is available in machine-readable form.)

adopted as a mean magnitude the median of measurements and do not provide luminosity amplitudes. Note that for $\mathrm{RR}_{d}$ variables the same observables and the period ratios, $P_{0} / P_{1}$, are listed in Table 6. For these stars we did not perform the analysis of pulsational parameters in the $U$-band, because the $U$-band light curves are not well sampled.

Figures 1 and 2 show the IS and a zoom of the position of RRLs in the $V,(B-V)$ CMD, respectively. Red and green circles display fundamental and first-overtone RRLs. Orange triangles and gray circles mark double-mode pulsators and 
Table 4

Photometry of the Carina Variable Stars

\begin{tabular}{|c|c|c|c|c|c|c|c|c|c|c|c|}
\hline HJD & $U$ & $\sigma_{U}$ & HJD & $B$ & $\sigma_{B}$ & HJD & $V$ & $\sigma_{V}$ & HJD & $I$ & $\sigma_{I}$ \\
\hline \multicolumn{12}{|l|}{ V7 } \\
\hline 53351.7001 & 21.50 & 0.03 & 51552.5736 & 21.33 & 0.02 & 51552.5669 & 20.90 & 0.02 & 53351.7698 & 20.38 & 0.02 \\
\hline 53351.7129 & 21.64 & 0.03 & 51552.5854 & 21.40 & 0.02 & 51552.5786 & 20.90 & 0.01 & 53351.7745 & 20.40 & 0.02 \\
\hline 53351.7256 & 21.59 & 0.03 & 51552.5971 & 21.43 & 0.02 & 51552.5904 & 20.94 & 0.02 & 53351.7791 & 20.38 & 0.02 \\
\hline 53351.7387 & 21.59 & 0.04 & 51552.6089 & 21.43 & 0.02 & 51552.6021 & 20.92 & 0.01 & 53351.7838 & 20.37 & 0.02 \\
\hline 53351.7515 & 21.62 & 0.04 & 51552.6209 & 21.47 & 0.01 & 51552.6141 & 21.03 & 0.01 & 53351.7885 & 20.43 & 0.02 \\
\hline 53351.7642 & 21.68 & 0.03 & 51552.6327 & 21.49 & 0.02 & 51552.6259 & 20.99 & 0.02 & 53351.7931 & 20.35 & 0.02 \\
\hline 53359.7729 & 21.39 & 0.03 & 51552.6445 & 21.53 & 0.02 & 51552.6378 & 21.04 & 0.01 & 53351.7978 & 20.43 & 0.02 \\
\hline 53359.7858 & 21.14 & 0.02 & 51552.6562 & 21.53 & 0.02 & 51552.6495 & 21.04 & 0.02 & 53359.6762 & 20.39 & 0.01 \\
\hline 53359.7992 & 20.75 & 0.02 & 51552.6681 & 21.56 & 0.02 & 51552.6613 & 21.08 & 0.01 & 53359.6808 & 20.39 & 0.02 \\
\hline \multirow[t]{2}{*}{53359.8121} & 20.45 & 0.02 & 51552.6798 & 21.54 & 0.02 & 51552.6731 & 21.04 & 0.02 & 53359.6855 & 20.34 & 0.02 \\
\hline & & & 51552.6916 & 21.58 & 0.02 & 51552.6849 & 21.10 & 0.02 & 53359.6901 & 20.36 & 0.01 \\
\hline
\end{tabular}

(This table is available in its entirety in machine-readable form.)

candidate Blazhko RRLs, while cyan symbols show the location of AC variable stars. Yellow and blue squares represent RGB and LPV variables, while black stars display the locations of the EBs (see Table 2). Finally, magenta and black pentagons show the RRLs and ACs in the catalog of VMC13 and not in common with our catalog. Blue and red lines display the theoretical IS boundaries predicted by radial nonlinear convective pulsation models for ACs (Fiorentino et al. 2006) and RRLs (Marconi et al. 2015, hereafter M2015). Theoretical predictions were computed assuming a metal abundance of $Z=0.0001$ (ACs) and of $Z=0.001$ (RRLs). Theory was transformed into the observational plane using the bolometric corrections and the color-temperature relations provided by Cardelli et al. (1989). We also adopted a true distance modulus of $\mu=(20.09 \pm 0.07)$ mag (Coppola et al. 2013) and a reddening of $E(B-V)=0.03 \mathrm{mag}$ (Monelli et al. 2003). The agreement between theory and observations appears, within the errors, quite good both for RRLs and ACs. In the above figures the two bright $\mathrm{RR}_{a b}$ stars (V158, V182) were defined as peculiar RRLs in Paper VI. We also note the presence of two $\mathrm{RR}_{a b}(\mathrm{~V} 170, \mathrm{~V} 227)$ that appear anomalously blue. These stars have poorly sampled light curves and uncertain pulsational parameters. In particular, the number of measurements we have for the variable V170 is too small to fit the light curve and to estimate the luminosity amplitude (see the Appendix for details). These four stars are labeled and marked in Figure 2 with blue crosses.

Moreover, we identified 101 new SX Phe. Their pulsation properties, their light curves and their position in the $V,(B-V)$ CMD are discussed in Section 5.

\section{PULSATION PROPERTIES}

Figure 3 shows the $B V I$ light curves for fundamental (left) and first overtone (middle) RRLs plus ACs (right). The different data sets were plotted with different colors. The light curves of $\mathrm{RR}_{d}$ variables are plotted in Figure 4. The complete atlas of light curves, including the $U$-band data, is available in the online edition of the paper.

\subsection{Bailey Diagram}

The left panels of Figure 5 display the Bailey diagramamplitude versus period-for RRLs. From top to bottom the different panels show amplitudes in the $B$ (top), $V$ (middle) and $I$ (bottom) bands. The Bailey diagram is a very good diagnostic for splitting fundamental and first overtones. The transition period between low-amplitude, short-period $\mathrm{RR}_{c}$ and largeamplitude, long-period $\mathrm{RR}_{a b}$ is located around $\log P \sim-0.35 /$ $-0.30 \quad(P \sim 0.45-0.5$ days $)$. The $\mathrm{RR}_{a b}$ display a steady decrease in amplitude when moving toward longer periods. On the other hand, the $\mathrm{RR}_{c}$ show a "bell-shaped" (Bono et al. 1997a) or a "hairpin" (Kunder et al. 2013) distribution. The Bailey diagram is also used to constrain the Oosterhoff class (see, e.g., Bono et al. 1997b) of stellar systems hosting sizable samples of RRLs. The middle panel shows the comparison between $\mathrm{RR}_{a b}$ in the $V$-band with the empirical relations provided by (Clement \& Shelton 1999, dashed lines) and by Cacciari et al. (2005, black solid line). The former relations are based on both OoI and OoII GCs, while the latter are only for OoI GCs. The comparison indicates that Carina can be classified either as an OoI or as an Oosterhoff intermediate system, since for periods longer than $\log P>-0.2$ there is a group of $\mathrm{RR}_{a b}$ that, at fixed period, attains larger luminosity amplitudes. On the other hand, the amplitudes typical of $\mathrm{RR}_{c}$ variables in OoII GCs (Kunder et al. 2013, blue solid line) agree quite well the current data.

In Paper VI we classified Carina, on the basis of the mean period of fundamentalized RRLs, as an OoII system. Indeed, we found $\left\langle P_{\mathrm{RR}}\right\rangle=0.60 \pm 0.01$ days $(\sigma=0.07)$. Including the new discovered $\mathrm{RR}_{a b}$ variables we find that the mean period of fundamental RRLs is $\left\langle P_{\mathrm{RR}}\right\rangle=0.637 \pm 0.006$ days $(\sigma=0.05)$. This estimate suggests that Carina is closer to an OoII stellar systems, since OoII GCs show mean fundamental periods of $\langle P\rangle \sim 0.65$ days, while the OoI GCs show shorter periods, namely $\langle P\rangle \sim 0.55$ days. We have also computed the ratio between the number of $\mathrm{RR}_{c}$ and the total number of RRLs, and we found $N_{c} /\left(N_{a b}+N_{c}\right) \sim 0.14$, i.e., a fraction of $\mathrm{RR}_{c}$ variables that is more typical of OoI $(\sim 17 \%)$ than OoII ( $\sim 4 \%$ ) GCs. The above evidence indicates that the Oosterhoff classification of Carina depends on the adopted diagnostic. This means that the Oosterhoff classification should be cautiously treated, since it depends on the adopted diagnostic on the completeness and size of the RRL sample and on the morphology of the HB (Fiorentino et al. 2015a).

The data plotted in Figure 5 also show mixed-mode pulsators, the so-called $\mathrm{RR}_{d}$ variables. These are radial variables oscillating simultaneously in at least two different pulsation modes. The $\mathrm{RR}_{d}$ typically oscillate in the first overtone and in the fundamental mode, and the former mode is usually stronger than the latter (e.g., Smith 2006), but there are 
Table 5

Pulsation Properties of Carina Variable Stars

\begin{tabular}{|c|c|c|c|c|c|c|c|c|c|c|c|}
\hline ID & Type & Epoch $^{\mathrm{a}}$ & Period (day) & $\langle U\rangle^{\mathrm{b}}$ & $\langle B\rangle^{\mathrm{b}}$ & $\langle V\rangle^{\mathrm{b}}$ & $\langle I\rangle^{\mathrm{b}}$ & $A(U)$ & $A(B)$ & $A(V)$ & $A(I)$ \\
\hline 3 & $\mathrm{ab}$ & 52351.2771 & 0.5786581 & $21.30^{\mathrm{c}}$ & 21.127 & 20.771 & 20.210 & $\ldots$ & 1.256 & 1.012 & 0.637 \\
\hline 4 & $\mathrm{c}$ & 51548.6519 & 0.3973509 & 21.243 & 20.995 & 20.685 & 20.234 & 0.513 & 0.663 & 0.565 & 0.356 \\
\hline 7 & $a b$ & 51548.7025 & 0.6033121 & $21.43^{\mathrm{c}}$ & 21.104 & 20.729 & 20.212 & $\ldots$ & 1.278 & 1.087 & 0.685 \\
\hline 10 & $\mathrm{ab}$ & 51548.7378 & 0.5845143 & 21.158 & 21.070 & 20.724 & 20.203 & 0.712 & 1.367 & 1.095 & 0.690 \\
\hline 14 & $\mathrm{AC}$ & 51548.9240 & 0.4766975 & 20.510 & 20.265 & 19.994 & 19.507 & 0.838 & 0.886 & 0.745 & 0.469 \\
\hline 17 & $\mathrm{~EB}$ & 51548.5693 & 0.3933393 & $22.03^{c}$ & 20.968 & 19.606 & 17.705 & $\ldots$ & 0.092 & 0.086 & 0.059 \\
\hline 22 & $a b$ & 51549.0012 & 0.6380630 & 21.209 & 21.155 & 20.746 & 20.133 & 0.757 & 0.709 & 0.518 & 0.326 \\
\hline 24 & $a b$ & 51548.5844 & 0.6181987 & $21.48^{\mathrm{c}}$ & 21.088 & 20.730 & 20.126 & $\ldots$ & 0.909 & 0.723 & 0.456 \\
\hline 25 & $\mathrm{Bl}$ & 52351.7201 & 0.5956510 & $21.20^{\mathrm{c}}$ & 21.077 & 20.718 & 20.128 & $\ldots$ & 0.993 & 0.856 & 0.539 \\
\hline 27 & $\mathrm{AC}$ & 51549.1519 & 1.0203875 & $20.23^{c}$ & 19.817 & 19.376 & 18.820 & $\ldots$ & 1.118 & 0.971 & 0.612 \\
\hline 29 & $\mathrm{AC}$ & 51549.2591 & 0.7178952 & 19.297 & 19.283 & 19.013 & 18.608 & 1.152 & 1.015 & 0.811 & 0.511 \\
\hline 30 & $a b$ & 51548.7682 & 0.6188105 & $21.57^{\mathrm{c}}$ & 21.179 & 20.774 & 20.136 & $\ldots$ & 0.893 & 0.699 & 0.441 \\
\hline 31 & $\mathrm{~B} 1$ & 51548.7359 & 0.6457949 & $21.39^{\mathrm{c}}$ & 21.154 & 20.750 & 20.093 & $\ldots$ & 0.598 & 0.468 & 0.295 \\
\hline 32 & $\mathrm{ab}$ & 51548.6507 & 0.6150517 & $21.44^{\mathrm{c}}$ & 21.108 & 20.723 & 20.096 & $\ldots$ & 0.875 & 0.743 & 0.468 \\
\hline 33 & $\mathrm{AC}$ & 51549.1187 & 0.5836254 & $20.47^{\mathrm{c}}$ & 20.055 & 19.735 & 19.155 & $\ldots$ & 0.779 & 0.645 & 0.406 \\
\hline 34 & $\mathrm{ab}$ & 51548.8657 & 0.5869516 & $21.44^{\mathrm{c}}$ & 21.108 & 20.767 & 20.281 & $\ldots$ & 1.281 & 0.993 & 0.625 \\
\hline 40 & $\mathrm{c}$ & 51548.6092 & 0.3926228 & 21.183 & 21.125 & 20.781 & 20.223 & 0.429 & 0.525 & 0.441 & 0.278 \\
\hline 41 & $\mathrm{AC}$ & 51548.5461 & 1.0381530 & $19.77^{\mathrm{c}}$ & 19.393 & 19.091 & 18.577 & $\ldots$ & 1.552 & 1.215 & 0.765 \\
\hline 43 & $\mathrm{c}$ & 51548.9153 & 0.2992499 & $21.31^{\mathrm{c}}$ & 20.945 & 20.741 & 20.353 & $\ldots$ & 0.567 & 0.477 & 0.300 \\
\hline 44 & $a b$ & 52351.3257 & 0.6264277 & $20.89^{\mathrm{c}}$ & 21.109 & 20.740 & 20.160 & $\ldots$ & 1.181 & 0.963 & 0.607 \\
\hline 47 & $\mathrm{c}$ & 51548.7090 & 0.3237674 & $21.26^{\mathrm{c}}$ & 20.996 & 20.762 & 20.355 & $\cdots$ & 0.760 & 0.619 & 0.390 \\
\hline 49 & $a b$ & 51548.8332 & 0.6815073 & $21.30^{\mathrm{c}}$ & 21.106 & 20.690 & 20.052 & $\ldots$ & 0.537 & 0.419 & 0.264 \\
\hline 57 & $a b$ & 51548.5449 & 0.6129016 & 21.294 & 21.195 & 20.783 & 20.185 & 0.854 & 0.854 & 0.671 & 0.423 \\
\hline 58 & $a b$ & 52351.7078 & 0.6194599 & 21.119 & 21.079 & 20.690 & 20.075 & 0.688 & 0.839 & 0.644 & 0.406 \\
\hline 60 & $a b$ & 51549.4418 & 0.6094132 & 21.341 & 21.148 & 20.746 & 20.157 & 0.539 & 1.016 & 0.808 & 0.509 \\
\hline 61 & $\mathrm{Bl}$ & 51548.8037 & 0.6213493 & $21.28^{\mathrm{c}}$ & 21.070 & 20.674 & 20.088 & $\ldots$ & 0.936 & 0.795 & 0.501 \\
\hline 65 & $a b$ & 51548.6491 & 0.6517109 & 21.171 & 21.079 & 20.707 & 20.109 & 1.242 & 1.069 & 0.922 & 0.581 \\
\hline 67 & $a b$ & 51548.9560 & 0.60372385 & 21.064 & 21.100 & 20.726 & 20.136 & 0.763 & 0.856 & 0.716 & 0.451 \\
\hline 68 & $a b$ & 51548.5512 & 0.6787359 & $21.43^{\mathrm{c}}$ & 21.158 & 20.721 & 20.091 & $\ldots$ & 0.430 & 0.352 & 0.222 \\
\hline 73 & $a b$ & 51548.5572 & 0.5695182 & $21.11^{\mathrm{c}}$ & 21.100 & 20.756 & 20.184 & $\ldots$ & 1.324 & 1.134 & 0.715 \\
\hline 75 & $a b$ & 52345.7593 & 0.5912161 & 21.209 & 21.192 & 20.746 & 20.164 & 0.757 & 0.682 & 0.538 & 0.339 \\
\hline 77 & $\mathrm{Bl}$ & 51548.6782 & 0.6043226 & $21.38^{\mathrm{c}}$ & 21.101 & 20.730 & 20.138 & $\ldots$ & 1.170 & 0.837 & 0.527 \\
\hline 84 & $a b$ & 51548.9285 & 0.6166815 & $21.01^{\mathrm{c}}$ & 21.082 & 20.722 & 20.107 & $\ldots$ & 0.954 & 0.795 & 0.501 \\
\hline 85 & $\mathrm{ab}$ & 51548.6088 & 0.6405248 & 21.078 & 21.068 & 20.666 & 20.065 & 0.594 & 0.889 & 0.654 & 0.412 \\
\hline 87 & $\mathrm{AC}$ & 51548.9360 & 0.8556130 & 19.144 & 18.975 & 18.732 & 18.353 & 0.526 & 0.644 & 0.478 & 0.301 \\
\hline 90 & $a b$ & 51548.6598 & 0.6313625 & $21.46^{\mathrm{c}}$ & 21.135 & 20.724 & 20.145 & $\ldots$ & 0.807 & 0.636 & 0.401 \\
\hline 91 & $a b$ & 51548.7635 & 0.7180705 & 21.164 & 21.105 & 20.684 & 20.023 & 0.600 & 0.751 & 0.579 & 0.365 \\
\hline 92 & $a b$ & 51548.7778 & 0.6301283 & 21.112 & 21.157 & 20.737 & 20.124 & 0.677 & 0.760 & 0.619 & 0.390 \\
\hline 105 & $a b$ & 51548.4033 & 0.6323014 & $20.99^{c}$ & 21.108 & 20.699 & 20.085 & $\ldots$ & 0.834 & 0.680 & 0.428 \\
\hline 115 & $\mathrm{AC}$ & 51549.4635 & 1.0109789 & 19.284 & 19.016 & 18.703 & 18.196 & 0.547 & 0.841 & 0.691 & 0.435 \\
\hline 116 & $a b$ & 51548.2506 & 0.6833133 & $21.15^{\mathrm{c}}$ & 21.157 & 20.758 & 20.194 & $\ldots$ & 0.938 & 0.727 & 0.458 \\
\hline 122 & $a b$ & 51548.8260 & 0.6314692 & 21.096 & 21.066 & 20.673 & 20.065 & 0.905 & 0.865 & 0.734 & 0.462 \\
\hline 123 & $a b$ & 51548.5547 & 0.6749693 & $21.35^{\mathrm{c}}$ & 21.079 & 20.674 & 20.065 & $\ldots$ & 0.829 & 0.689 & 0.434 \\
\hline 124 & $a b$ & 51548.9470 & 0.5917211 & $21.34^{\mathrm{c}}$ & 21.043 & 20.672 & 20.139 & $\ldots$ & 1.117 & 0.899 & 0.566 \\
\hline 125 & $a b$ & 51548.4329 & 0.5940963 & $20.94^{\mathrm{c}}$ & 20.964 & 20.641 & 20.073 & $\cdots$ & 1.332 & 1.091 & 0.688 \\
\hline 126 & $\mathrm{Bl}$ & 51548.5952 & 0.5570972 & $21.23^{\mathrm{c}}$ & 21.072 & 20.741 & 20.160 & $\ldots$ & 1.070 & 0.917 & 0.578 \\
\hline 127 & $\mathrm{~B} 1$ & 51548.3900 & 0.6262952 & $21.22^{\mathrm{c}}$ & 21.140 & 20.744 & 20.163 & $\ldots$ & 0.694 & 0.506 & 0.319 \\
\hline 129 & $\mathrm{AC}$ & 51549.2201 & 0.6301768 & 19.477 & 19.368 & 19.107 & 18.698 & 0.788 & 1.006 & 0.802 & 0.505 \\
\hline 133 & $a b$ & 52351.8941 & 0.6123127 & 21.398 & 21.160 & 20.776 & 20.237 & 1.463 & 1.011 & 0.813 & 0.512 \\
\hline 135 & $a b$ & 51548.9530 & 0.5909193 & $21.30^{\mathrm{c}}$ & 20.982 & 20.624 & 20.091 & $\ldots$ & 1.213 & 0.945 & 0.596 \\
\hline 136 & $a b$ & 51548.1625 & 0.631613 & $21.24^{\mathrm{c}}$ & 21.099 & 20.691 & 20.064 & $\ldots$ & 0.731 & 0.575 & 0.362 \\
\hline 138 & $a b$ & 51548.3027 & 0.6392611 & $21.12^{\mathrm{c}}$ & 21.110 & 20.704 & 20.094 & $\ldots$ & 0.691 & 0.549 & 0.346 \\
\hline 141 & $a b$ & 51548.3418 & 0.6353340 & $21.20^{\mathrm{c}}$ & 21.143 & 20.737 & 20.144 & $\cdots$ & 0.688 & 0.532 & 0.335 \\
\hline 142 & $\mathrm{c}$ & 51548.4286 & 0.3635433 & $21.05^{\mathrm{c}}$ & 21.004 & 20.734 & 20.254 & $\ldots$ & 0.677 & 0.548 & 0.345 \\
\hline 143 & $a b$ & 51548.6506 & 0.6095789 & 21.297 & 21.063 & 20.674 & 20.116 & 1.336 & 0.934 & 0.763 & 0.481 \\
\hline 144 & $\mathrm{c}$ & 51548.9292 & 0.3933566 & 21.086 & 20.969 & 20.661 & 20.153 & 0.414 & 0.545 & 0.427 & 0.269 \\
\hline 148 & $\mathrm{c}$ & 51548.7335 & 0.3266545 & $21.01^{\mathrm{c}}$ & 20.656 & 20.444 & 20.084 & $\ldots$ & 0.701 & 0.592 & 0.373 \\
\hline 149 & $\mathrm{AC}$ & 51548.5879 & 0.9177072 & $20.96^{\mathrm{c}}$ & 20.444 & 20.077 & 19.488 & $\cdots$ & 1.074 & 0.888 & 0.560 \\
\hline 151 & $\mathrm{c}$ & 51548.8155 & 0.3418011 & $21.35^{\mathrm{c}}$ & 21.125 & 20.842 & 20.374 & $\ldots$ & 0.584 & 0.465 & 0.293 \\
\hline 153 & $a b$ & 51548.4220 & 0.6603692 & 21.076 & 21.072 & 20.674 & 20.083 & 0.717 & 0.670 & 0.539 & 0.339 \\
\hline 158 & $a b$ & 51548.5230 & 0.6324566 & 20.902 & 20.785 & 20.330 & 19.642 & 0.715 & 0.625 & 0.458 & 0.288 \\
\hline 159 & $a b$ & 51548.5223 & 0.5751520 & $20.94^{\mathrm{c}}$ & 21.033 & 20.702 & 20.171 & $\ldots$ & 1.164 & 0.976 & 0.615 \\
\hline 164 & $a b$ & 51548.3880 & 0.6339196 & $21.38^{\mathrm{c}}$ & 21.095 & 20.718 & 20.104 & $\ldots$ & 0.709 & 0.569 & 0.359 \\
\hline 165 & $a b$ & 51548.6742 & 0.5790136 & $20.57^{c}$ & 21.057 & 20.751 & 20.223 & $\ldots$ & 1.315 & 1.081 & 0.681 \\
\hline
\end{tabular}


Table 5

(Continued)

\begin{tabular}{|c|c|c|c|c|c|c|c|c|c|c|c|}
\hline ID & Type & Epoch $^{\mathrm{a}}$ & Period (day) & $\langle U\rangle^{\mathrm{b}}$ & $\langle B\rangle^{\mathrm{b}}$ & $\langle V\rangle^{\mathrm{b}}$ & $\langle I\rangle^{\mathrm{b}}$ & $A(U)$ & $A(B)$ & $A(V)$ & $A(I)$ \\
\hline 170 & $a b$ & 51543.0033 & $0.59^{\mathrm{d}}$ & $21.06^{\mathrm{c}}$ & $21.04^{\mathrm{c}}$ & $20.79^{c}$ & $20.26^{\mathrm{c}}$ & $\ldots$ & $\ldots$ & $\ldots$ & $\ldots$ \\
\hline 171 & $a b$ & 51542.6010 & $0.66^{\mathrm{d}}$ & $21.48^{\mathrm{c}}$ & $21.14^{\mathrm{c}}$ & $20.76^{\mathrm{c}}$ & $20.11^{\mathrm{c}}$ & $\ldots$ & $\ldots$ & $\ldots$ & $\ldots$ \\
\hline 173 & RGB & 51549.1135 & 0.657779 & $20.79^{c}$ & 19.573 & 18.542 & $17.25^{\mathrm{c}}$ & $\ldots$ & 0.095 & 0.094 & \\
\hline 174 & $\mathrm{ab}$ & 51548.5789 & 0.6531203 & $21.22^{\mathrm{c}}$ & 21.175 & 20.756 & 20.152 & $\ldots$ & 0.507 & 0.417 & 0.263 \\
\hline 175 & $\mathrm{c}$ & 51548.8389 & 0.3923778 & $21.23^{\mathrm{c}}$ & 21.068 & 20.739 & 20.263 & $\ldots$ & 0.532 & 0.454 & 0.286 \\
\hline 176 & $a b$ & 51549.4881 & 0.764565 & $21.33^{c}$ & 21.160 & 20.715 & 20.061 & $\ldots$ & 0.264 & 0.212 & 0.133 \\
\hline 178 & $\mathrm{AC}$ & 51549.0958 & 1.0155700 & $19.25^{\mathrm{c}}$ & 19.535 & 19.207 & 18.768 & $\ldots$ & 1.512 & 1.244 & 0.784 \\
\hline 179 & $\mathrm{ab}$ & 51548.7597 & 0.6637945 & $21.26^{\mathrm{c}}$ & 21.155 & 20.719 & 20.081 & $\ldots$ & 0.247 & 0.196 & 0.124 \\
\hline 181 & $\mathrm{c}$ & 51549.0010 & 0.2794913 & $21.16^{\mathrm{c}}$ & 20.996 & 20.781 & 20.350 & $\ldots$ & 0.095 & 0.076 & 0.048 \\
\hline 182 & $a b$ & 51548.1454 & 0.7889722 & $20.48^{\mathrm{c}}$ & 20.588 & 20.178 & 19.571 & $\ldots$ & 0.380 & 0.323 & 0.204 \\
\hline 183 & $a b$ & 51548.7509 & 0.612302 & $21.21^{\mathrm{c}}$ & 20.963 & 20.596 & 20.013 & $\ldots$ & 0.615 & 0.508 & 0.320 \\
\hline 184 & c & 51548.4830 & 0.3951290 & 21.165 & 21.011 & 20.714 & 20.213 & 0.499 & 0.539 & 0.422 & 0.266 \\
\hline 185 & $a b$ & 51548.5511 & 0.6209146 & $21.31^{\mathrm{c}}$ & 21.097 & 20.731 & 20.155 & $\ldots$ & 0.871 & 0.678 & 0.427 \\
\hline 187 & $\mathrm{AC}$ & 51549.0083 & 0.9502923 & $19.42^{\mathrm{c}}$ & 19.440 & 19.166 & 18.674 & $\ldots$ & 1.389 & 1.154 & 0.727 \\
\hline 188 & $a b$ & 51548.6637 & 0.5973773 & $21.19^{\mathrm{c}}$ & 21.019 & 20.665 & 20.143 & $\ldots$ & 1.290 & 1.127 & 0.710 \\
\hline 189 & $a b$ & 51548.8546 & 0.7023692 & $21.22^{\mathrm{c}}$ & 21.028 & 20.639 & 20.044 & $\ldots$ & 0.771 & 0.647 & 0.407 \\
\hline 190 & $\mathrm{AC}$ & 51549.2923 & 1.1647103 & 19.622 & 19.478 & 19.137 & 18.547 & 0.848 & 1.546 & 1.294 & 0.815 \\
\hline 191 & $a b$ & 51548.7011 & 0.6502894 & 21.066 & 21.091 & 20.686 & 20.114 & 0.633 & 0.711 & 0.561 & 0.354 \\
\hline 193 & $\mathrm{AC}$ & 51549.1506 & 0.4263580 & $19.68^{c}$ & 19.530 & 19.284 & 18.900 & $\ldots$ & 0.172 & 0.131 & 0.082 \\
\hline 194 & $\mathrm{FV}$ & 51549.2016 & 0.2645828 & $16.32^{\mathrm{c}}$ & 16.076 & 15.879 & 15.525 & $\ldots$ & 0.133 & 0.123 & 0.072 \\
\hline 195 & $a b$ & 51548.5576 & 0.6189680 & $21.18^{\mathrm{c}}$ & 21.140 & 20.769 & 20.143 & $\ldots$ & 0.646 & 0.483 & 0.304 \\
\hline 196 & $a b$ & 51548.2819 & 0.665989 & 21.213 & 21.081 & 20.676 & 20.132 & 0.740 & 0.500 & 0.410 & 0.258 \\
\hline 197 & $\mathrm{c}$ & 51548.7640 & 0.2961717 & $21.24^{\mathrm{c}}$ & 20.959 & 20.739 & 20.331 & $\ldots$ & 0.390 & 0.312 & 0.197 \\
\hline 199 & $a b$ & 51548.1840 & 0.769925 & 20.976 & 20.999 & 20.574 & 19.942 & 0.322 & 0.485 & 0.406 & 0.256 \\
\hline 200 & $a b$ & 51548.5254 & 0.6373468 & $21.24^{\mathrm{c}}$ & 21.156 & 20.738 & 20.101 & $\ldots$ & 0.359 & 0.289 & 0.182 \\
\hline 201 & $a b$ & 51548.5885 & 0.7222435 & $21.32^{\mathrm{c}}$ & 21.133 & 20.730 & 20.084 & $\ldots$ & 0.762 & 0.632 & 0.398 \\
\hline 203 & $\mathrm{AC}$ & 51549.2587 & 0.9398875 & 19.924 & 19.820 & 19.427 & 18.838 & 0.736 & 1.052 & 0.812 & 0.511 \\
\hline 204 & $a b$ & 51548.8631 & 0.6332687 & $21.40^{\mathrm{c}}$ & 21.121 & 20.725 & 20.094 & $\ldots$ & 0.654 & 0.545 & 0.344 \\
\hline 205 & $\mathrm{AC}$ & 51549.1382 & 0.3833676 & 20.189 & 19.891 & 19.668 & 19.281 & 0.399 & 0.156 & 0.145 & 0.091 \\
\hline 206 & $\mathrm{Bl}$ & 51548.5382 & 0.587812 & $21.48^{\mathrm{c}}$ & 21.069 & 20.700 & 20.113 & $\ldots$ & 1.192 & 1.053 & 0.664 \\
\hline 208 & $a b$ & 52352.0043 & 0.656594 & $21.39^{c}$ & 21.125 & 20.721 & 20.073 & $\ldots$ & 0.619 & 0.484 & 0.305 \\
\hline 214 & $\mathrm{~B} 1$ & 52352.3784 & 0.6391863 & $21.17^{\mathrm{c}}$ & 21.031 & 20.674 & 20.077 & $\ldots$ & 1.041 & 0.753 & 0.475 \\
\hline 216 & $\mathrm{AC}$ & 51549.6831 & 1.079230 & $19.32^{\mathrm{c}}$ & 19.258 & 18.853 & 18.201 & $\ldots$ & 0.427 & 0.303 & 0.191 \\
\hline 217 & $\mathrm{AC}$ & 51549.7742 & 0.9109459 & $18.71^{\mathrm{c}}$ & 18.864 & 18.600 & 18.156 & $\ldots$ & 0.839 & 0.676 & 0.425 \\
\hline 218 & $\mathrm{AC}$ & 52351.7154 & 1.0086934 & $20.44^{\mathrm{c}}$ & 20.141 & 19.742 & 19.224 & $\ldots$ & 0.571 & 0.485 & 0.305 \\
\hline 219 & $\mathrm{AC}$ & 52352.9984 & 1.365517 & $19.58^{\mathrm{c}}$ & 19.277 & 18.879 & 18.252 & $\ldots$ & 0.476 & 0.387 & 0.244 \\
\hline 220 & EB & 51548.7085 & 0.37264174 & $15.59^{c}$ & 15.364 & 14.681 & 13.872 & $\ldots$ & 0.733 & 0.750 & 0.663 \\
\hline 221 & WUma & 51549.0298 & 1.783454 & $22.01^{\mathrm{c}}$ & 20.551 & 19.228 & $17.43^{\mathrm{c}}$ & $\ldots$ & 0.165 & 0.150 & $\ldots$ \\
\hline 222 & LP & 51519.2828 & 329 & $21.70^{\mathrm{c}}$ & $19.35^{\mathrm{c}}$ & $17.42^{\mathrm{c}}$ & $15.66^{\mathrm{c}}$ & $\ldots$ & $\ldots$ & $\ldots$ & $\ldots$ \\
\hline 223 & $\mathrm{ab}$ & 52352.8734 & 0.660937 & $21.24^{\mathrm{c}}$ & 21.105 & 20.715 & 20.061 & $\ldots$ & 0.309 & 0.257 & 0.162 \\
\hline 224 & LP & 52401.0706 & 685 & $20.10^{\mathrm{c}}$ & $21.05^{\mathrm{c}}$ & $20.47^{\mathrm{c}}$ & $19.83^{c}$ & $\ldots$ & $\ldots$ & $\ldots$ & $\ldots$ \\
\hline 226 & $a b$ & 51549.3101 & 0.669846 & $21.12^{\mathrm{c}}$ & 21.086 & 20.698 & 20.082 & $\ldots$ & 0.432 & 0.322 & 0.203 \\
\hline 227 & $a b$ & 51550.7387 & 0.5594254 & $\ldots$ & 21.265 & 20.940 & 20.274 & $\ldots$ & 0.876 & 0.607 & 0.382 \\
\hline 228 & $a b$ & 51548.2822 & 0.6290089 & $20.86^{\mathrm{c}}$ & 21.082 & 20.713 & 20.083 & $\ldots$ & 0.600 & 0.500 & 0.315 \\
\hline 229 & $a b$ & 51548.3681 & 0.6626030 & $21.02^{\mathrm{c}}$ & 21.085 & 20.681 & 20.072 & $\ldots$ & 0.449 & 0.342 & 0.235 \\
\hline 230 & $\mathrm{AC}$ & 52351.3174 & 1.0025734 & $19.73^{c}$ & 19.580 & 19.2546 & 18.663 & $\ldots$ & 1.353 & 1.074 & 0.677 \\
\hline 231 & $\mathrm{~EB}$ & 51549.5699 & 2.3831781 & $21.19^{c}$ & $21.36^{\mathrm{c}}$ & $21.23^{\mathrm{c}}$ & $20.97^{\mathrm{c}}$ & $\ldots$ & $\ldots$ & $\ldots$ & $\ldots$ \\
\hline 232 & $\mathrm{~EB}$ & 51548.7853 & 0.4142902 & $17.28^{\mathrm{c}}$ & $16.44^{\mathrm{c}}$ & $15.44^{\mathrm{c}}$ & $14.14^{\mathrm{c}}$ & $\ldots$ & $\ldots$ & $\ldots$ & $\ldots$ \\
\hline 233 & EB & 51548.8868 & 0.6378688 & $22.48^{\mathrm{c}}$ & $22.37^{\mathrm{c}}$ & $22.26^{\mathrm{c}}$ & $22.14^{\mathrm{c}}$ & $\ldots$ & $\ldots$ & $\ldots$ & $\ldots$ \\
\hline 234 & EB & 51548.4601 & 0.5274709 & $24.44^{\mathrm{c}}$ & $22.59^{\mathrm{c}}$ & $21.14^{\mathrm{c}}$ & $18.99^{c}$ & $\ldots$ & $\ldots$ & $\ldots$ & $\ldots$ \\
\hline 235 & $\mathrm{~EB}$ & 51548.6759 & 0.6539230 & $18.54^{\mathrm{c}}$ & $18.43^{\mathrm{c}}$ & $17.74^{\mathrm{c}}$ & $16.89^{c}$ & $\ldots$ & $\ldots$ & $\ldots$ & $\ldots$ \\
\hline 236 & $\mathrm{~EB}$ & 51548.7125 & 0.3747540 & $22.35^{\mathrm{c}}$ & $22.41^{\mathrm{c}}$ & $22.35^{\mathrm{c}}$ & 22.32 & $\ldots$ & $\ldots$ & $\ldots$ & $\ldots$ \\
\hline 237 & $\mathrm{~EB}$ & 51548.7915 & 0.4083364 & $22.03^{c}$ & 22.003 & 21.761 & $21.49^{c}$ & $\ldots$ & 0.323 & 0.314 & $\ldots$ \\
\hline
\end{tabular}

Notes.

${ }^{\mathrm{a}}$ Epoch of maximum light.

b Intensity-averaged magnitudes.

${ }^{\mathrm{c}}$ Median of individual magnitude measurements.

${ }^{\mathrm{d}}$ Not enough data to fit a light curve, uncertain parameters.

exceptions (Clementini et al. 2004). On the other hand, Classical Cepheids display a wide range of mixed-mode pulsators among the overtones and fundamental mode (Soszynski et al. 2008; Soszyñski et al. 2010), suggesting that surface gravity and effective temperature might play fundamental roles in driving the occurrence of such a phenomenon. Figure 6 shows the comparison in the Bailey diagram between the current observations and predicted amplitudes. Pulsation 
Table 6

Pulsation Properties of Carina $\mathrm{RR}_{d}$ Variable Stars

\begin{tabular}{|c|c|c|c|c|c|c|c|c|c|c|c|c|}
\hline ID & Epoch & $\begin{array}{l}P_{0} \\
\text { (days) }\end{array}$ & $\begin{array}{l}P_{1} / P_{0} \\
\text { (days) }\end{array}$ & $\begin{array}{c}\langle B\rangle \\
(\mathrm{mag})\end{array}$ & $\begin{array}{c}\langle V\rangle \\
(\mathrm{mag})\end{array}$ & $\begin{array}{c}\langle I\rangle \\
(\mathrm{mag})\end{array}$ & $\begin{array}{l}A\left(B_{0}\right) \\
(\mathrm{mag})\end{array}$ & $\begin{array}{l}A\left(B_{1}\right) \\
(\mathrm{mag})\end{array}$ & $\begin{array}{l}A\left(V_{0}\right) \\
(\mathrm{mag})\end{array}$ & $\begin{array}{l}A\left(V_{1}\right) \\
(\mathrm{mag})\end{array}$ & $\begin{array}{l}A\left(I_{0}\right) \\
(\mathrm{mag})\end{array}$ & $\begin{array}{l}A\left(I_{1}\right) \\
(\mathrm{mag}\end{array}$ \\
\hline V11 & 51548.9191 & 0.543689 & 0.745854 & 21.080 & 20.766 & 20.247 & 0.548 & 0.341 & 0.451 & 0.339 & 0.28 & 0.21 \\
\hline V26 & 51548.9291 & 0.562177 & 0.745703 & 20.990 & 20.670 & 20.162 & 0.604 & 0.488 & 0.610 & 0.395 & 0.39 & 0.25 \\
\hline V89 & 51548.6377 & 0.519403 & 0.746522 & 21.102 & 20.770 & 20.222 & 0.408 & 0.384 & 0.524 & 0.278 & 0.33 & 0.17 \\
\hline V192 & 51549.0958 & 0.541694 & 0.748661 & 21.030 & 20.720 & 20.252 & 0.753 & 0.525 & 0.600 & 0.345 & 0.38 & 0.22 \\
\hline V198 & 51548.6238 & 0.530551 & 0.745534 & 21.058 & 20.710 & 20.192 & 0.664 & 0.323 & 0.506 & 0.334 & 0.32 & 0.21 \\
\hline V225 & 52351.8655 & 0.57688 & 0.74610 & 20.960 & 20.641 & 20.147 & 0.776 & 0.363 & 0.575 & 0.324 & 0.36 & 0.20 \\
\hline
\end{tabular}

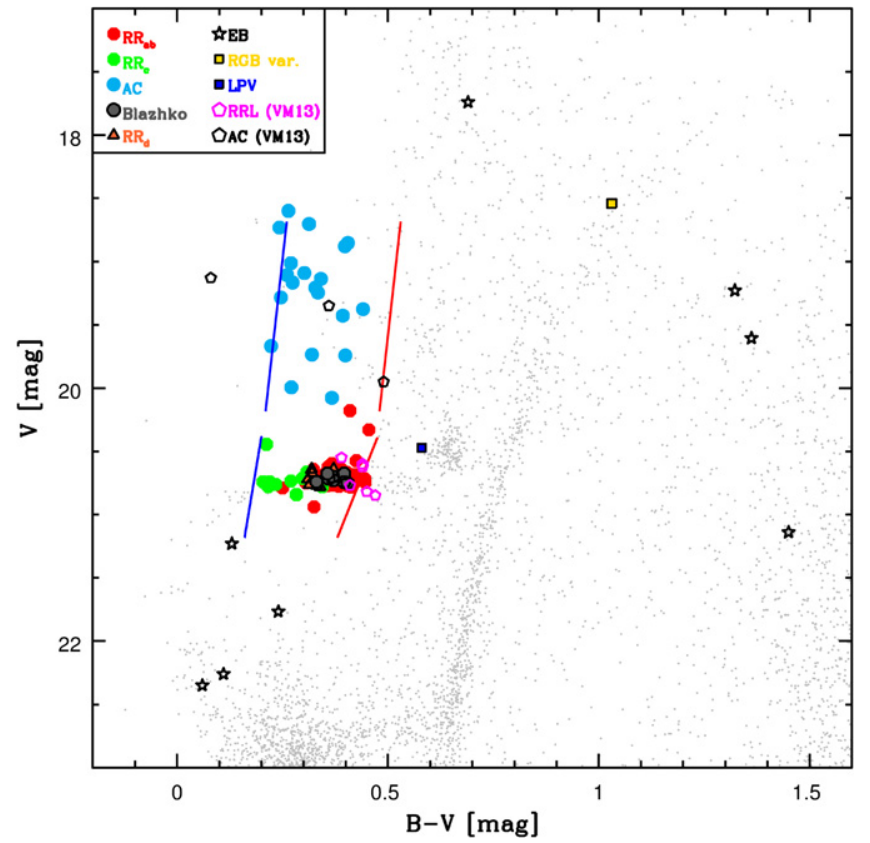

Figure 1. Position of variable stars in $V,(B-V) \mathrm{CMD}$. Red and green circles display fundamental and first-overtone RRLs. Orange triangles and gray circles mark double-mode pulsators and candidate Blazhko RRLs. Cyan symbols show ACs. Yellow and blue squares display candidate RGB and LPV variables, while black stars show the EB variables. Two EBs, not members of Carina, fall outside the plot limits; their colors and magnitudes are given in Table 5.

prescriptions rely on a large set of RRL models recently provided by M2015. The black solid and dotted lines display predicted amplitudes for the sequence of metal-poor $(Z=0.0001, Y=0.245)$ models constructed by assuming a stellar mass of $0.80 M_{\odot}$ and two different luminosity levels. The black solid line shows predictions for the Zero-AgeHorizontal-Branch (ZAHB) luminosity level $\left(\log \left(L / L_{\odot}\right)\right.$ $=1.76)$, while the dotted line for a brighter luminosity level $\left(\log \left(L / L_{\odot}\right)=1.86\right)$. The purple lines display the same predictions, but for a slightly more metal-rich chemical composition $\left(Z=0.0003, Y=0.245 ; M=0.716 M_{\odot}, \log \right.$ $\left(L / L_{\odot}\right)=1.72$ and 1.82$)$.

The predicted amplitudes appear to be slightly larger, at fixed pulsation period, when compared with observations. This is a limit in the current theoretical framework, since the amplitudes are tightly correlated with the efficiency of convective transport. In passing we note that the comparison between predicted and observed luminosity amplitudes is

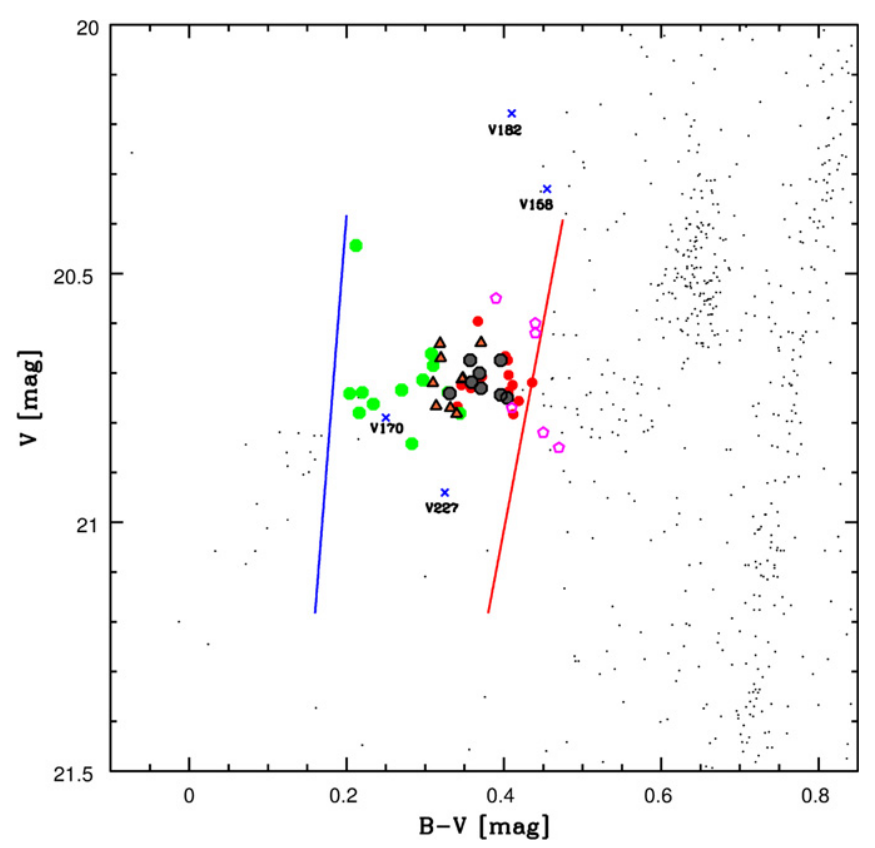

Figure 2. Same as Figure 1, but zoomed on the CMD region located around the RR Lyrae instability strip.

hampered by the current theoretical uncertainties in the treatment of the time dependent convective transport. We current adopt a mixing length parameter of $\alpha=1.5$ (see Marconi et al. 2011). Larger values cause a steady decrease in the luminosity amplitude (see Di Criscienzo et al. 2004). Indeed, observed amplitudes attain, at fixed period smaller amplitudes. This applies to both fundamental and first overtone variables. Moreover, we are assuming that observed light curves have a good sampling around the phases of minimum and maximum light. However, the comparison shows two interesting features. (i) The predicted amplitudes for FO pulsators show a larger dependence on metal content than fundamental pulsators. (ii) The regular pulsators display, at fixed pulsation period, a small spread in amplitudes. The current predictions suggest that their evolutionary status is quite homogenous, since they appear to be located to the ZAHB luminosity level.

Carina was previously known to host six $\mathrm{RR}_{d}$ variables: V11, V26, V89, V192, V198, V207 (D03); in the current analysis we discovered other three double-mode pulsators: V74, V210, and V225. To further understand their nature, and in particular to properly define the location of $\mathrm{RR}_{d}$ pulsators in the Bailey diagram, we estimated both primary (first overtone) 

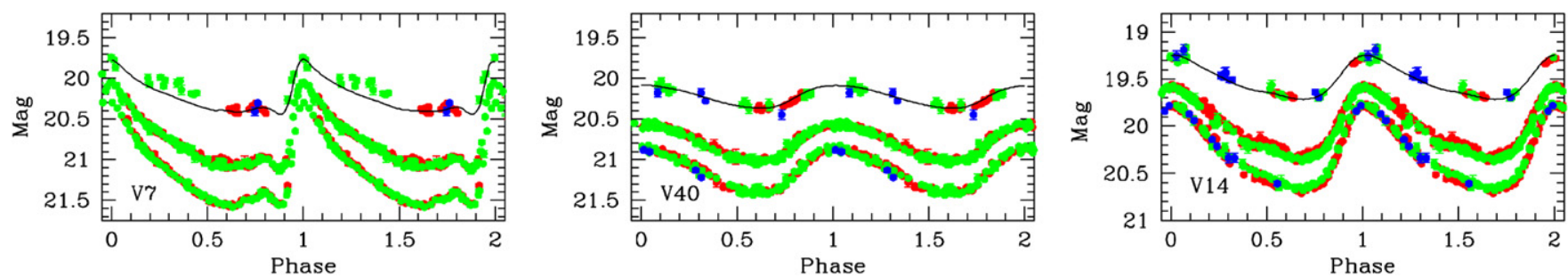

Figure 3. $B V I$ light curves for selected Carina variables. We selected one $\mathrm{RR}_{a b}\left(\mathrm{~V} 7\right.$, left panel), one $\mathrm{RR}_{c}(\mathrm{~V} 40$, middle panel), and one $\mathrm{AC}$ (V14, right panel). Red, green, blue, and yellow filled circles display different data sets: MOSAIC2@CTIO, WFI@MPI/ESO, Tek2K-I@CTIO, and FORS1@ESO/VLT. Black lines are the fits of the light curves. For the $I$ band they are the template light curves obtained by properly scaling the $V$-band light curve according to the procedure described in Section 2. The $B V I$ plus the $U$-band light curves for the the entire sample of variables are given in the electronic edition of the journal.

(The complete figure set (15 images) is available.)
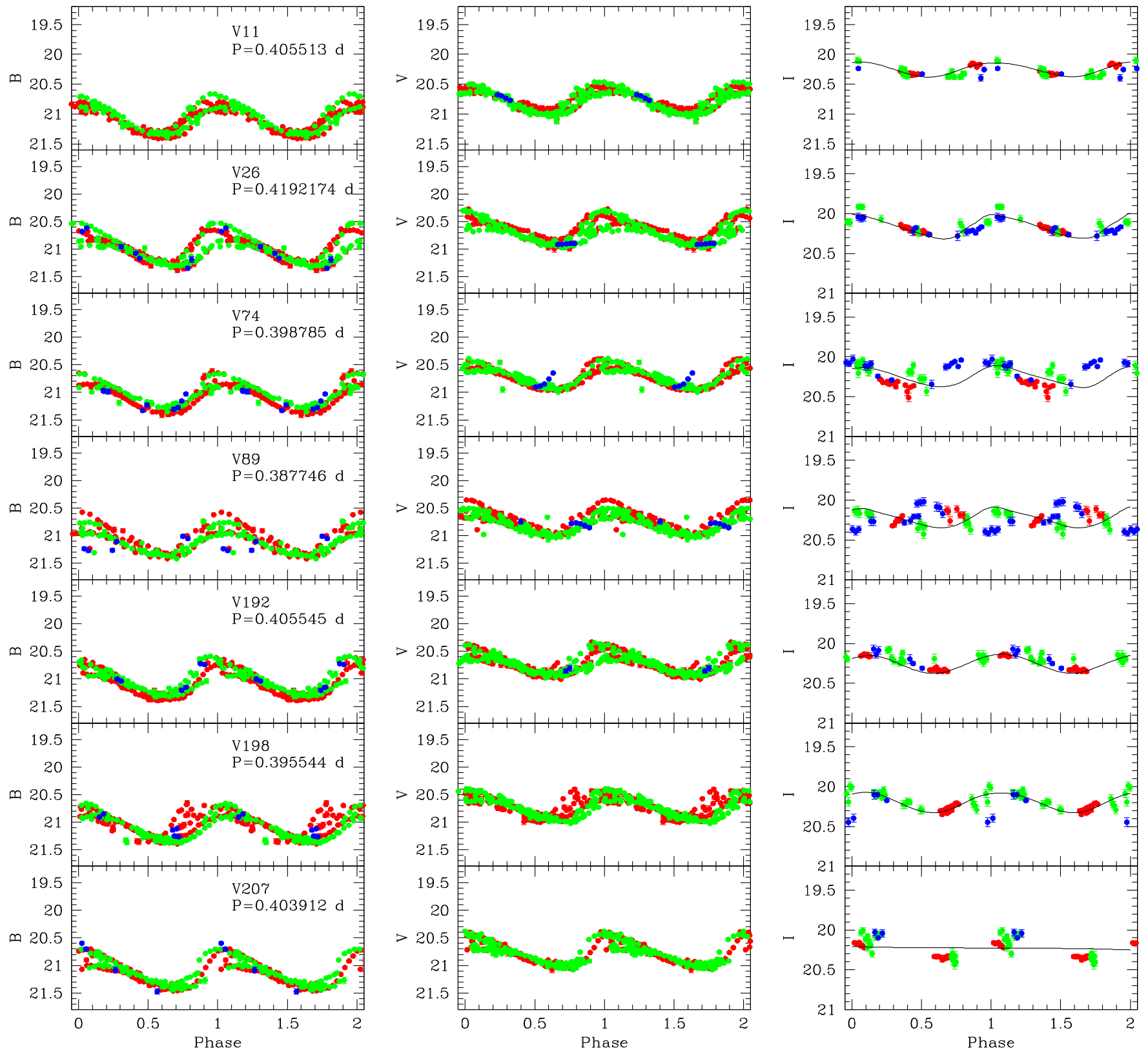

Figure 4. Same as Figure 3, but for the $B V I$ light curves of the nine $\mathrm{RR}_{d}$ stars in our sample. 

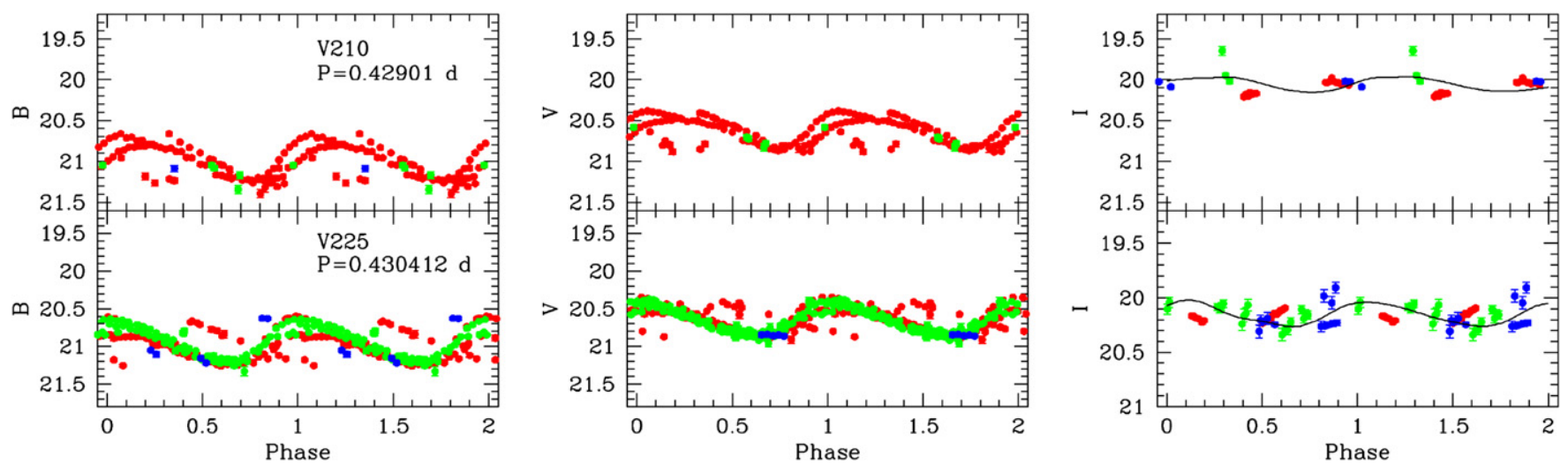

Figure 4. (Continued.)

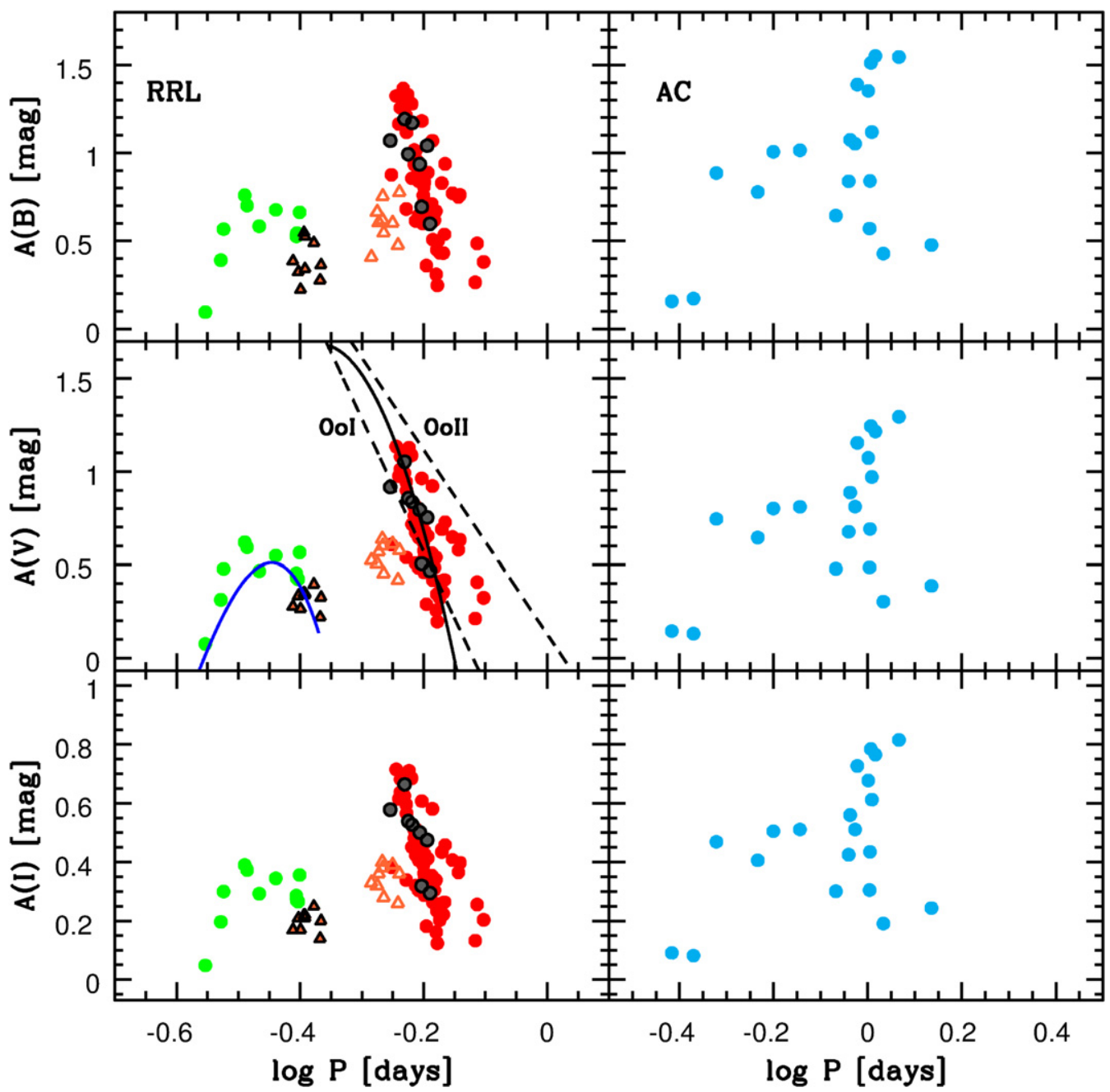

Figure 5. From top to bottom Bailey diagram in $B$ (top), $V$ (middle), and $I$ band (bottom) for Carina RRLs (left) and ACs (right). The dashed lines plotted in the middle left panel display OoI and OoII relations for fundamental pulsators in Galactic globular clusters according to Clement \& Shelton (1999), while the black solid line shows the relation for OoI cluster according to Cacciari et al. (2005). The blue solid line shows the relation for OoII first overtone cluster variables provided by Kunder et al. (2013). Symbols are the same as in Figure 1. Note that double-mode variables have been plotted using periods and amplitudes of both primary (first overtone, filled orange triangles) and secondary (fundamental, empty orange triangles) components.

and secondary (fundamental) periods and decomposed their light curves. Indeed, the quality of the photometry allowed us to estimate not only the "global luminosity amplitude," but also the amplitude of both fundamental (open orange triangles) and first overtone mode (filled orange triangles). Table 6 gives from left to right their periods, mean magnitudes and amplitudes in the $B V I$ bands. To our knowledge this is the first time in which we can associate to the two modes of double-mode variables their individual luminosity amplitudes. The data plotted in the left panels of Figure 5 indicate that the primary components 


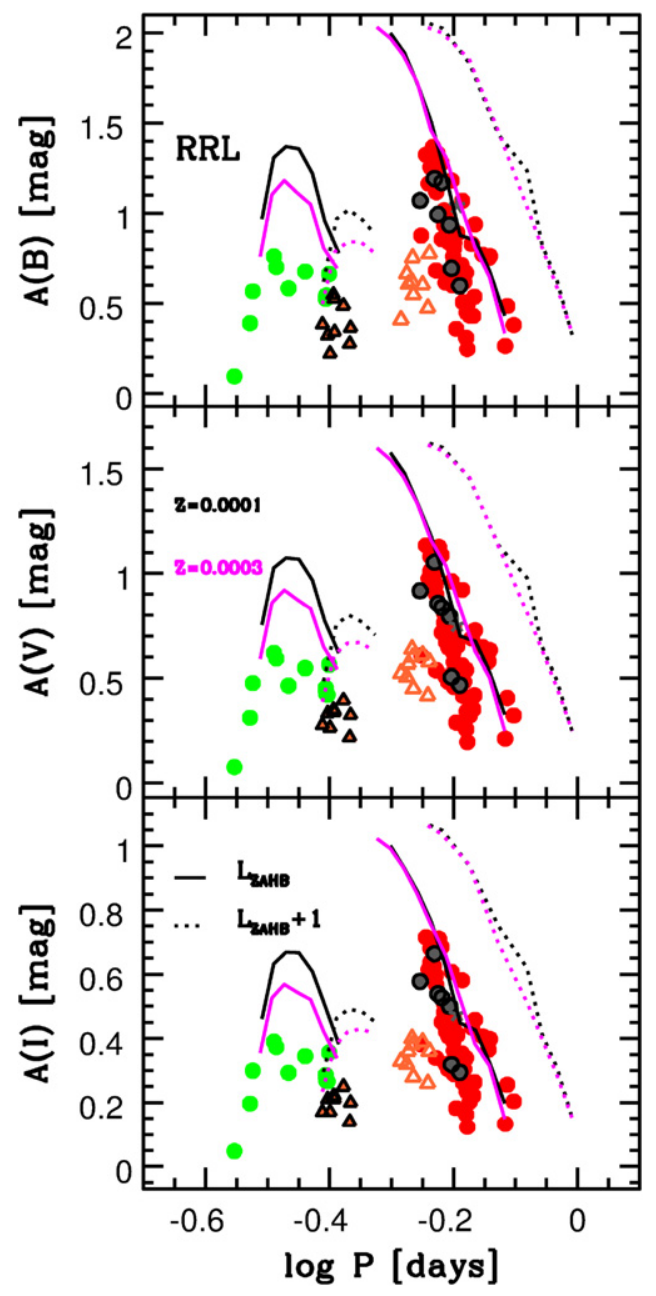

Figure 6. Same as left panels of Figure 5, but the comparison is between observations and predicted luminosity amplitude provided by M2015. The solid lines display predicted amplitude for $\mathrm{F}$ and FO models constructed by assuming a stellar mass of $M=0.80 M_{\odot}$ a metal-poor chemical composition $(Z=0.0001, Y=0.245)$ and Zero-Age-Horizontal-Branch luminosity level $\left(\log \left(L \biguplus_{\odot}\right)=1.76\right)$. The black dotted lines display the same predictions, but for models constructed by assuming a brighter luminosity level $\left(\log \left(L / L_{\odot}\right)\right.$ $=1.86)$. The purple lines display the same pulsation predictions, but for pulsation models constructed by assuming a less metal-poor chemical composition $(Z=0.0003, Y=0.245)$.

(first overtone) are located in the long period tale $(\log P \sim$ -0.4 ) of single mode first overtone variables. Moreover, the secondary components (fundamental) are located in the short period tale $(\log P \sim-0.25)$ of single mode fundamental variables. This evidence is further supported by their mean colors. The mean color of $\mathrm{RR}_{d}$ is systematically redder $(B-V$ $\sim 0.33 \mathrm{mag}$ ) than the color range covered by $\mathrm{RR}_{c}$ variables $(0.2 \leqslant B-V \leqslant 0.35 \mathrm{mag})$ and systematically bluer than the typical color range of $\operatorname{RR}_{a b}$ variables $(0.3 \leqslant B-V \leqslant$ $0.5 \mathrm{mag})$.

The pulsation and evolutionary status of the $\mathrm{RR}_{d}$ variables depends on their evolutionary direction and on their position in the so-called OR region (Bono et al. 1997b). In this context it is worth mentioning that RRLs in dwarf spheroidal galaxies appear to lack High Amplitude Short Period (HASP) fundamental variables (Stetson et al. 2014b; Fiorentino et al. 2015a), thus resembling Oosterhoff II globular clusters in the Milky Way (Bono et al. 1997b).
Table 7

Pulsation Properties for the Nine Extra-tidal Variables (Six RRLs, Four ACs) Identified by VM13

\begin{tabular}{lcccccc}
\hline \hline ID & Type & $\begin{array}{c}P \\
(\text { days })\end{array}$ & $\begin{array}{c}(B) \\
(\mathrm{mag})\end{array}$ & $\begin{array}{c}(V) \\
(\mathrm{mag})\end{array}$ & $\begin{array}{c}A(B) \\
(\mathrm{mag})\end{array}$ & $\begin{array}{c}A(V) \\
(\mathrm{mag})\end{array}$ \\
\hline RRL-1 & $\mathrm{ab}$ & 0.629 & 20.94 & 20.55 & 0.55 & 0.45 \\
RRL-2 & $\mathrm{ab}$ & 0.523 & 21.04 & 20.60 & 0.83 & 0.65 \\
RRL-3 & $\mathrm{ab}$ & 0.544 & 21.06 & 20.62 & 0.56 & 0.46 \\
RRL-4 & $\mathrm{c}$ & 0.204 & 21.32 & 20.85 & 0.17 & 0.24 \\
RRL-5 & $\mathrm{c}$ & 0.107 & 21.27 & 20.82 & 0.14 & 0.27 \\
RRL-38 & $\mathrm{c}$ & 0.189 & 21.18 & 20.77 & 0.41 & 0.31 \\
AC-1 & $\ldots$ & 0.186 & 20.44 & 19.95 & 0.21 & 0.20 \\
AC-9 & $\ldots$ & 0.476 & 19.21 & 19.13 & 0.36 & 0.55 \\
AC-10 & $\ldots$ & 0.163 & 19.71 & 19.35 & 0.18 & 0.32 \\
\hline
\end{tabular}

The occurrence of a good sample of $\mathrm{RR}_{d}$ variables in Carina seems to suggest that this region of the Bailey diagram might be populated by mixed-mode variables. Obviously, the occurrence of $\mathrm{RR}_{d}$ variables depends on the topology of the IS, but also on the evolutionary properties (extent in temperature of the so-called blue hook) and, in particular, on the occurrence of the hysteresis mechanism (van Albada \& Baker 1971; Bono et al. 1995; Fiorentino et al. 2015a; Marconi et al. 2015) when moving from more metal-poor to more metalrich stellar structures.

The Blazhko RRLs plotted in Figure 5 also appear to be located in a very narrow period range. No firm conclusion can be reached concerning the distribution in the Bailey diagram of Blazhko RRLs, since the sample size is quite limited and also because the Blazhko cycle is poorly sampled.

The above findings further support the crucial role played by cluster and galactic RRLs to constrain the topology of the IS and to investigate the evolutionary and pulsation status of exotic objects like $\mathrm{RR}_{d}$ and Blazhko RRLs.

The right panels of Figure 5 show the distribution of Carina ACs in the same Bailey diagrams as the RRLs. Their properties have already been discussed in Paper VI. We confirm the separation at $\log P \sim-0.1$ between long-period and highamplitude with short-period and low-amplitude ACs. In passing we note that three out of the 20 ACs have periods around one day, thus further supporting the need for data sets covering large time intervals to remove the one-day alias.

Note that in the current analysis of evolved variables we did not include the six RRLs (three $\mathrm{RR}_{c}$, three $\mathrm{RR}_{a b}$ ) and the three ACs recently detected by VM13 outside the tidal radius of Carina. The reasons are the following. The three $\mathrm{RR}_{c}$ variables attain periods that are systematically shorter $(-1.0 \lesssim \log P \lesssim$ -0.25 ) that typical $\mathrm{RR}_{c}$ variables (see Table 7), but their mean $B-V$ colors are typical of $\mathrm{RR}_{a b}$ variables. The same outcome applies to the three $\mathrm{RR}_{a b}$ variable, and indeed they are located in the short period range of fundamental pulsators, but their $B-V$ colors are typical of objects located close to the red edge of the IS. Moreover, the newly identified ACs have periods that are systematically shorter $(-0.8 \lesssim \log P \lesssim-0.7)$ than the typical Carina ACs.

\subsection{Petersen Diagram}

The top panel of Figure 7 displays the position of the Carina $\mathrm{RR}_{d}$ variables in the Petersen diagram, i.e., the first-overtoneto-fundamental period ratio $\left(P_{1} / P_{0}\right)$ versus the fundamental period. The data in this panel also show the comparison with 


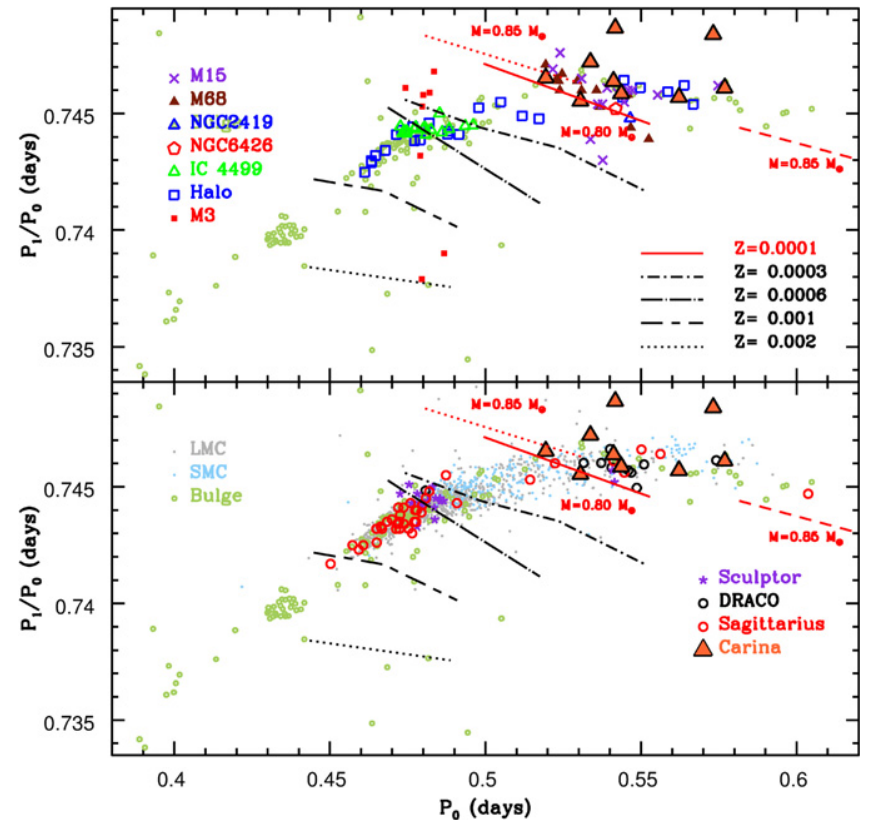

Figure 7. Top: comparison in the Petersen diagram between Galactic (halo, bulge, globular clusters), and Carina (orange triangles) $\mathrm{RR}_{d}$ variables. Pulsation predictions (M2015) for different chemical compositions (see the labeled values) are plotted with different lines. The stellar mass of the pulsation models for the most metal-poor chemical composition are also labeled. Bottom: same as the top, but the comparison is with $\mathrm{RR}_{d}$ variables in nearby dwarf spheroidals and irregulars. The $\mathrm{RR}_{d}$ of the Galactic bulge are also plotted.

$\mathrm{RR}_{d}$ variables identified in Galactic globulars (see labeled names) and in the Galactic field (Halo: blue squares; Bulge: small green circles). Table 8 gives from left to right the name of the stellar system, the number of $\mathrm{RR}_{d}$ variables, the reference for the $\mathrm{RR}_{d}$ data, the mean metallicity, and the reference for the metallicity estimate. The data plotted in this figure display several interesting features.

The period ratio shows a steady decrease when moving from more metal-poor to more metal-rich systems (see also Bragaglia et al. 2001). The largest values in the period ratio are attained in the Halo and in very metal-poor GCs, while the smallest values are attained in the Bulge. The trend with the metallicity was also suggested on both theoretical and empirical bases by Soszyński et al. (2011) and more recently by Soszyński et al. (2014). Note that the fraction of doublemode variables appears to be anti-correlated with the mean metal abundance, and indeed it increases from $0.5 \%$ in the Bulge to $4 \%$ in the LMC and to $10 \%$ in the SMC (Soszyński et al. 2011). The $\mathrm{RR}_{d}$ variables in Carina appear to follow a similar trend, since the current fraction is $\sim 10 \%$. Two $\mathrm{RR}_{d}$ variables (V192, V210) display period ratios that are systematically larger then period ratios in Galactic globulars, in dwarf galaxies, and in the Bulge. In passing we note that one $\mathrm{RR}_{d}$ in M68 (V36) and two in M15 (V51, V53) display period ratios that systematically smaller than the bulk of $\mathrm{RR}_{d}$ variables. The referee suggested the difference on a more quantitative basis. To avoid spurious fluctuations in the period range covered by the different data sets, we ranked the entire sample (398) as a function of the fundamental period. Then we estimated the running average by using a box including the first 40 objects in the list. We estimated the mean period ratio, the mean fundamental period, and the standard deviations of this subsample. We estimated the same quantities by moving one object in the ranked list until we took account of the last 40 objects in the sample. We performed several tests, changing both the number of objects included in the box and the number of stepping stars. The current findings are minimally affected by plausible variations. We found that the quoted five $\mathrm{RR}_{d}$ stars are located within $3 \sigma$ of the mean. The current statistics is too limited to claim solid evidence of discrepancy. Note that we double checked the photometric quality and coverage of the above five objects and we found that they are similar to the other canonical $\mathrm{RR}_{d}$ stars.

The period ratios display a larger spread when moving into the metal-poor regime (long fundamental periods). To define the trend on a more quantitative basis we adopted several sets of nonlinear, convective pulsation models (M2015). The adopted chemical compositions are labeled. The new set of RRL models relies on the theoretical framework outlined in Di Criscienzo et al. (2004) and Marconi et al. (2011), but on new evolutionary prescriptions for low-mass He burning models provided by (Pietrinferni et al. 2004) (BASTI data base, http:// albione.oa-teramo.inaf.it). The pulsation predictions plotted in this panel cover the so-called OR region, i.e., the region in which RRLs show a stable limit cycle in both the fundamental and the first overtone mode. This region is located between the blue edge of the fundamental mode and the red edge of the first overtone. The comparison between theory and observations indicates that an increase in the luminosity $\left(\log L / L_{\odot}=1.76\right.$, 1.86, dotted and dashed red lines) mainly causes, at fixed chemical composition $(Z=0.0001)$ and stellar mass $(M /$ $\left.M_{\odot}=0.85\right)$, a steady increase in the fundamental period, and in turn a decrease in the period ratio. Moreover, a decrease in stellar mass $\left(M / M_{\odot}=0.80\right.$, solid red line) at fixed chemical composition and luminosity level $\left(\log L / L_{\odot}=1.76\right)$ causes a systematic decrease in the period ratio and a moderate increase of the fundamental period. The above trends take into account a significant fraction of $\mathrm{RR}_{d}$ pulsators located in metal-poor GCs and in Carina $\mathrm{dSph}$. This indicates that $\mathrm{RR}_{d}$ in Carina have a metallicity of the order of $Z=0.0001$ and a mean stellar mass close to $0.85 M_{\odot}$. The pulsation masses are slightly larger than predicted by evolutionary models, but are within the current empirical and theoretical uncertainties.

The above findings further support the evidence that the old stellar component in Carina is quite metal-poor. This evidence is soundly supported by recent photometric and spectroscopic results by Monelli et al. (2014) and Fabrizio et al. (2015) suggesting a mean metal abundance for the old stellar component of $[\mathrm{Fe} / \mathrm{H}]=(-2.13 \pm 0.03 \pm 0.28)$ dex.

In this context it is worth mentioning that theoretical predictions for more metal-rich pulsation models (see the black lines and the labeled values) provide a sound explanation of the steady decrease in the period ratio of Bulge $\mathrm{RR}_{d}$ variables, i.e., the stellar systems with the broader metallicity distribution.

To further define the pulsation and evolutionary properties of Carina $\mathrm{RR}_{d}$ variables, the bottom panel of Figure 7 shows the same Petersen diagram, but the comparison is now extended to $\mathrm{RR}_{d}$ in nearby dwarf spheroidals (Draco, Sculptor, Sagittarius), in dwarf irregulars (LMC; Small Magellanic Cloud, SMC) and in the Bulge. The data plotted in this panel bring forward several interesting new findings.

The range in period ratios covered by $\mathrm{LMC} \mathrm{RR}_{d}$ is on average larger $\left(0.740 \leqslant P_{1} / P_{0} \leqslant 0.749\right)$ than the range of SMC $\mathrm{RR}_{d}$ variables. This evidence supports spectroscopic 
Table 8

Number of $\mathrm{RR}_{d}$ Hosted in the Stellar Systems Plotted in Figure 7 and Their References

\begin{tabular}{|c|c|c|c|c|}
\hline System & $N$ & Reference & {$[\mathrm{Fe} / \mathrm{H}]$} & Reference \\
\hline NGC 2419 & 1 & Clement et al. (1993) & $-2.20 \pm 0.09$ & Carretta et al. (2009) \\
\hline NGC 6426 & 1 & Clement et al. (1993) & $-2.33 \pm 0.15$ & Hatzidimitriou et al. (1999) \\
\hline LMC & 985 & Soszyński et al. (2009) & $-0.33 \pm 0.13$ & Romaniello et al. $(2008)^{\mathrm{a}}$ \\
\hline Bulge & 173 & Soszyński et al. (2014) & $-1.5 /-0.5$ & Zoccali et al. $(2008)^{\mathrm{b}}$ \\
\hline Halo (NSV 09295) & 1 & Garcia-Melendo \& Clement (1997) & -1.5 & Layden (1994) \\
\hline Halo (AQ Leo) & 1 & Clement et al. (1991) & -1.5 & Layden (1994) \\
\hline Halo (ASAS) & 32 & Pojmanski (2002) & -1.5 & Layden (1994) \\
\hline M3 & 8 & Clementini et al. (2004) & $-1.50 \pm 0.05$ & Carretta et al. (2009) \\
\hline IC4499 & 16 & Walker \& Nemec (1996) & $-1.62 \pm 0.09$ & Carretta et al. (2009) \\
\hline M68 & 12 & Walker (1994) & $-2.27 \pm 0.04$ & Carretta et al. (2009) \\
\hline M15 & 14 & Nemec (1985b) & $-2.33 \pm 0.02$ & Carretta et al. (2009) \\
\hline Draco & 10 & Nemec (1985a) & $-1.98 \pm 0.01$ & Kirby et al. (2013) \\
\hline
\end{tabular}

Notes. Columns 4 and 5 give the iron abundances of the hosting stellar system and their references.

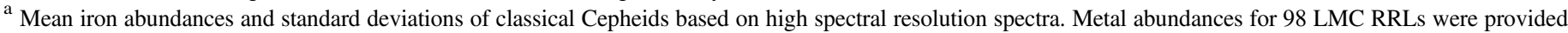

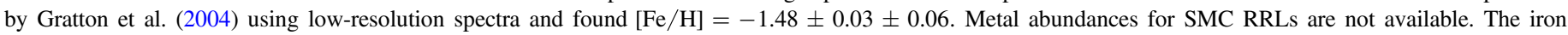
abundance of the single SMC GC (NGC 121) is $[\mathrm{Fe} / \mathrm{H}]=-1.19 \pm 0.12$ provided by Da Costa \& Hatzidimitriou (1998).

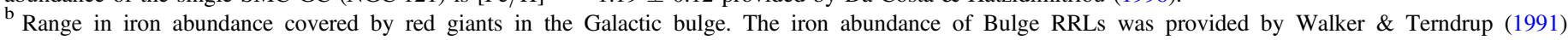
$[\mathrm{Fe} / \mathrm{H}]=-1.00 \pm 0.16$ using low-resolution spectra.

measurements of LMC RRLs suggesting metal abundances ranging from $[\mathrm{Fe} / \mathrm{H}]=-2.12$ to $-0.27 \mathrm{dex}$ (Gratton et al. 2004). The $\mathrm{SMC} \mathrm{RR}_{d}$ cover a slightly narrower period ratio range $\left(0.741 \leqslant P_{1} / P_{0} \leqslant 0.747\right.$ days $)$ but according to recent studies, and within current uncertainties affecting metallicity estimates for RRLs in these systems (Haschke et al. 2012) the metallicity spread for the old stellar populations in the two Clouds is similar.

The location of $\mathrm{RR}_{d}$ of Carina and Draco is the same in the Petersen diagram. Indeed current spectroscopic estimates, based on medium resolution spectra, provide a very metalpoor iron abundance $([\mathrm{Fe} / \mathrm{H}]=-1.92 \pm 0.01 \mathrm{dex}$, see Table 8$)$ also for Draco. The steady decrease in period ratio of $\mathrm{RR}_{d}$ in Sculptor is strongly supported by the recent spectroscopic measurements suggesting an iron abundance of $[\mathrm{Fe} / \mathrm{H}]=$ $-1.68 \pm 0.01 \mathrm{dex}$ (see Table 8). The empirical evidence concerning Sagittarius needs to be discussed in detail, because the periods and period ratios attain values that are on average smaller than for $\mathrm{RR}_{d}$ in other dSphs. Spectroscopic estimates based on high-resolution spectra by Carretta et al. (2010), suggest for Sagittarius a mean iron abundance, based on 27 $\mathrm{RGs}$, of $[\mathrm{Fe} / \mathrm{H}]=-0.62$ and individual values ranging from -1.0 to above solar. A smaller spread in iron abundance was also suggested by (Kunder \& Chaboyer 2008) using RR Lyrae properties. The spread in period ratios and the range in fundamental periods $(0.45 \leqslant P \leqslant 0.49$ days $)$ showed by Sagittarius RRLs soundly support the spectroscopic measurements and the similarity with LMC RRLs. This indicates that Sagittarius is a fundamental nearby laboratory to constrain the pulsation properties of metal-rich RRL in gas poor systems.

The $\mathrm{RR}_{d}$ in the Bulge (small green circles) display a clear overdensity for $P_{0} \sim 0.46$ days. This overdensity was explained by Soszyński et al. (2011) as the relic of a former dwarf galaxy that was captured by the Milky Way. More recent investigations based on a larger sample (28 versus 16$)$ indicate that they are distributed along a stream that crosses the Galactic

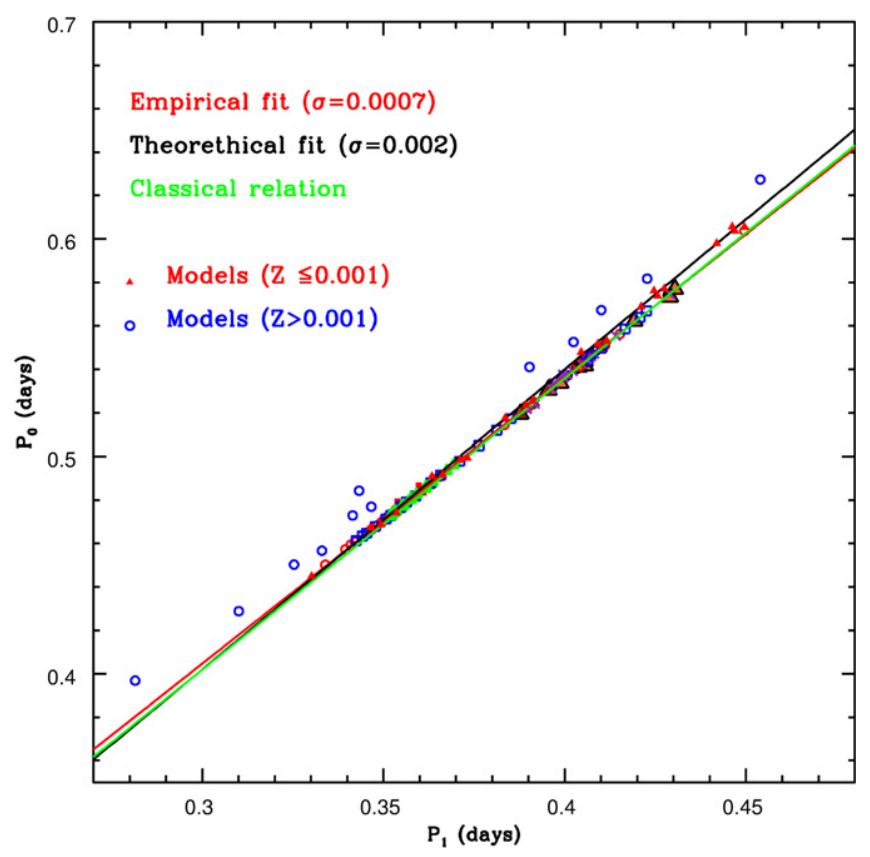

Figure 8. Correlation between fundamental and first overtone periods. Observed $\mathrm{RR}_{d}$ variables have been plotted using the same symbols of Figure 7. The red line shows the fit to the observed data, the black line shows the fit to the pulsation models and the green line shows the classical relation. Red triangles and blue dots display metal-poor and metal-rich pulsation models.

bulge almost vertically. Note that the comparison with theoretical predictions suggests, for the above stellar system, a metal-intermediate chemical composition $(\mathrm{Z}=0.001-0.002)$.

The $\mathrm{RR}_{d}$ also provide a unique opportunity to validate the current approach to fundamentalizing the first overtones. Whenever the sample of RRLs hosted in a stellar system is limited, fundamental and first overtone variables are treated as 


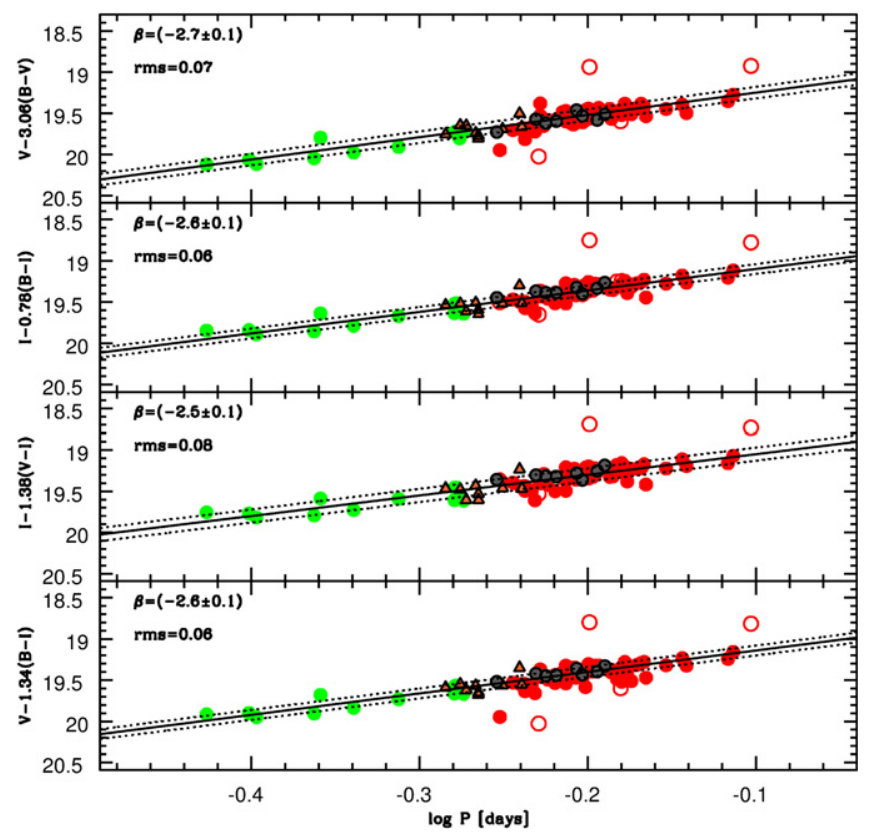

Figure 9. Observed optical PW relations. From top to bottom the different panels display the $B V$, the $B I$, the $V I$, and the $B V I$ relations. First overtone pulsators were fundamentalized. The symbols are the same as in Figure 1. The solid lines display the fit, while the dotted lines show the $1 \sigma$ difference. The standard deviations (rms), the coefficient of the logarithmic period, and their errors are also labeled. The two empty circles were not included in the estimate of the PW relations.

a single sample by transforming the periods of the first overtones into "equivalent" fundamental periods, using the relation $\log P_{\mathrm{F}}=\log P_{\mathrm{FO}}+0.127$. This assumption dates back to almost half a century and relies on the few $\mathrm{RR}_{d}$ variables known at that time (Sandage et al. 1981; Cox et al. 1983; Petersen 1991). The above constant period shift is further supported by the new theoretical scenario by M2015 and by the sizable sample of $\mathrm{RR}_{d}$ variables recently identified by large, dedicated photometric surveys.

In passing we note that this issue is far from being an academic dispute, since the same fix is also used to improve the precision of distance determinations based on both RRLs (Braga et al. 2015) and classical Cepheids (e.g., Marengo et al. 2010). Figure 8 shows fundamental versus first overtone periods $\left(P_{0}\right.$ versus $\left.P_{1}\right)$ for the entire sample of $\mathrm{RR}_{d}$ plotted in Figure 7 . The red line shows the fit to the empirical data. We found:

$$
P_{0}=0.0096+1.317 * P_{1} \text {. }
$$

The new estimate of both the slope and the zero-point soundly supports the old fix, and indeed the green line, showing the classical fix, agrees quite well with the new observations.

To further constrain the impact that the period ratios of $\mathrm{RR}_{d}$ variables have in constraining the pulsation properties of RRLs, we plotted in the same plane theoretical predictions for the "OR" regions adopted in Figure 7. The black solid line was estimated by considering only models more metal-poor than $Z=0.001$, i.e., a metallicity range similar to the observed one. The agreement is quite good over the period range. In passing we also note that when moving to more metal-rich models $(Z>0.001$, blue circles) the period increase is significantly larger in the fundamental period than in the first overtone one.
Table 9

Results of the Empirical Fit of Period-Wesenheit Relations $W=\alpha+\beta \log P_{\mathrm{F}}$

\begin{tabular}{lcc}
\hline \hline$\alpha$ & $\beta$ & $\mathrm{rms}$ \\
\hline$(18.98 \pm 0.03)$ & $W(B, V)=M_{V}-3.06(B-V)$ & 0.07 \\
& $(-2.7 \pm 0.1)$ & \\
$(18.84 \pm 0.03)$ & $(B, I)=M_{I}-0.78(B-I)$ & 0.06 \\
$(18.80 \pm 0.03)$ & $(-2.6 \pm 0.1)$ & 0.08 \\
& $(V, I)=M_{I}-1.38(V-I)$ & \\
$(18.88 \pm 0.03)$ & $(-2.5 \pm 0.1)$ & 0.06 \\
\hline
\end{tabular}

We still lack firm empirical evidence for such objects and it is not clear whether it is an observational bias or the consequence of an evolutionary property connected to the dependence of the HB morphology on the metal content.

\section{DISTANCE TO CARINA FROM OPTICAL PERIOD- WESENHEIT RELATIONS}

\subsection{Carina Distance Determination Based on the Empirical $P W B V$ Relation}

One of the most important tools for deriving distances from pulsating stars is the so called Wesenheit relation (see for example, van den Bergh 1975; Madore 1982) that is independent of reddening by definition, assuming that the ratio of total-to-selective absorption is fixed. This is a periodluminosity relation that includes a color term whose coefficient is the ratio between the total and the selective extinction coefficients. Figure 9 shows the observed PW relations and the empirical fit to the data (solid black lines) obtained by fundamentalizing the first overtone pulsators by using Equation (1). Dashed lines depict the dispersion of the above inferred relations. Results of these fits are listed in Table 9, where the zero-points, the slopes, and the dispersions of the relations are reported in the first three columns, respectively. In the fit determination we excluded stars outside $3 \sigma$ of the inferred empirical $B V$ Wesenheit relation. These stars are the two peculiar pulsators V158 and V182 and the stars V170 and V171 for which we have uncertain parameters (red open symbols).

Thanks to the use of the Fine Guidance Sensor on board the Hubble Space Telescope (HST), Benedict et al. (2011) provided accurate estimates of the trigonometric parallaxes for five field RRLs: SU Dra, XZ Cyg, RZ Cep, XZ Cyg, and RR Lyr. Using their data in Table 2, we derived the mean magnitude in the $B$ and $V$ bands from a fit with a spline under tension. We then calculated the absolute Wesenheit parameter for each star, $(W=\langle V\rangle-3.06(B-V)-\mu)$, where $\mu$ is the individual distance modulus based on the $H S T$ parallax. Individual mean magnitudes of the calibrating RRLs and their distances are listed in Table 10. We applied the individual calibrating RRL to the empirical PW relations (see Figure 9 and Table 9). Note that the calibrating RRLs cover a limited range in metallicity (from -1.80 to -1.41 dex, Benedict et al. 2011). The current theoretical predictions (see Section 4.2) suggest a mild dependence on the metal content. Therefore, we neglected the metallicity dependence of the calibrating RRLs. The data 
Table 10

Periods, $B$ and $V$ Mean Magnitudes, True Distance Moduli and Metallicity for the Five Field RRLs Stars by Benedict et al. (2011)

\begin{tabular}{lccccc}
\hline \hline Name & $\log P$ & $\langle B\rangle$ & $\langle V\rangle$ & $\mu$ & {$[\mathrm{Fe} / \mathrm{H}]$} \\
\hline RR Lyr & -0.24655 & $8.07 \pm 0.01$ & $7.75 \pm 0.01$ & $-1.41 \pm 0.13$ \\
SU Dra & -0.18018 & $9.71 \pm 0.01$ & $9.95 \pm 0.01$ & $9.35 \pm 0.07$ & $-1.80 \pm 0.2$ \\
UV Oct & -0.26552 & $10.13 \pm 0.01$ & $9.35 \pm 0.01$ & $8.87 \pm 0.13$ & $-1.47 \pm 0.11$ \\
XZ Cyg & -0.33107 & $9.92 \pm 0.01$ & $9.84 \pm 0.01$ & $8.98 \pm 0.22$ & $-1.44 \pm 0.2$ \\
RZ Cep & -0.38352 & $9.46 \pm 0.01$ & $8.03 \pm 0.16$ & $-1.77 \pm 0.2$ \\
\hline
\end{tabular}

Note. The distance moduli in this column are slightly different than those in Table 8 of Benedict et al. (2011) due to typographical errors in the paper. Iron abundances are on the Zinn \& West (1984) metallicity scale according to Benedict et al. (2011; see their Table 1).

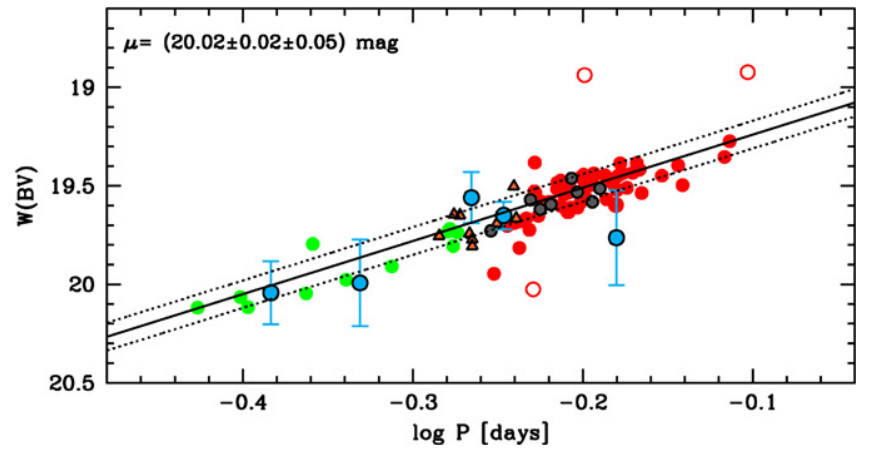

Figure 10. Same as the top panel of Figure 9, but with the the five field RRLs (light blue circles) for which trigonometric parallaxes have been estimated using FGS at $H S T$. The vertical error bars take account of the photometric error and of the uncertainty in distance. The object with the smallest error bar is RR Lyr itself. The true distance modulus based on the empirical slope and on the calibrating RRL is labeled.

plotted in Figure 10 show in the W-log $P$ plane the calibrating RRLs together with the Carina RRLs. The error bars of the calibrating RRLs take into account both the photometric errors and the uncertainties of the trigonometric parallaxes. A glance at the data discloses that only for RR Lyr is the precision of the absolute distance better than $1 \%$. Using all the calibrating RRLs we found a true distance modulus for Carina of $\mu=(20.02 \pm 0.02 \pm 0.05) \mathrm{mag}$. Note that for the above reasons the accuracy of the distance mainly depends on the accuracy of the data for RR Lyr.

\subsection{Carina Distance Determination Based on Theoretical $P W Z$ Relations}

To fully exploit the multiband photometry of Carina RRLs we also decided to use predicted PW relations. The new theoretical framework derived by M2015 indicates that the PW $(B V)$ relation is independent of the metal content. This is a very positive feature for two different reasons: (a) the PW $(B V)$ can be applied to estimate individual distances of field RRLs for which the metal content is not available; (b) the PW $(B V)$ is particularly useful to estimate distances of RRLs in nearby dwarf galaxies, since they typically cover a broad range in iron content. We applied the predicted relation to Carina RRLs and we found a true distance modulus of $\mu=(20.08 \pm 0.007 \pm$ $0.07) \mathrm{mag}$. The distance determination has been estimated using the predicted relation for fundamental pulsators. The number of $\mathrm{RR}_{c}$ variables in Carina is modest (12) and they have been fundamentalized. As noted in the previous section in the current distance determination we did not consider stars outside $3 \sigma$ of the inferred empirical Wesenheit $(B V)$ relation. The above distance agrees, within the errors, quite well with the
Table 11

Carina True Distance Moduli Based on Different Diagnostics

\begin{tabular}{llll}
\hline \hline$\mu$ & $E(B-V)$ & Method & Reference \\
\hline $20.06 \pm 0.12$ & 0.025 & PL (DC) & Mateo et al. (1998) \\
$20.10 \pm 0.12$ & 0.03 & PLC & D03 \\
$20.00 \pm 0.10$ & 0.03 & PLA & D03 \\
$20.10 \pm 0.04$ & 0.03 & FOBE & D03 \\
$20.11 \pm 0.13$ & 0.06 & TRGB & Pietrzyński et al. (2009) \\
$20.17 \pm 0.10$ & 0.063 & PL (DC) & Vivas \& Mateo (2013) \\
$20.09 \pm 0.07$ & 0.03 & PLW & Paper VI \\
\hline
\end{tabular}

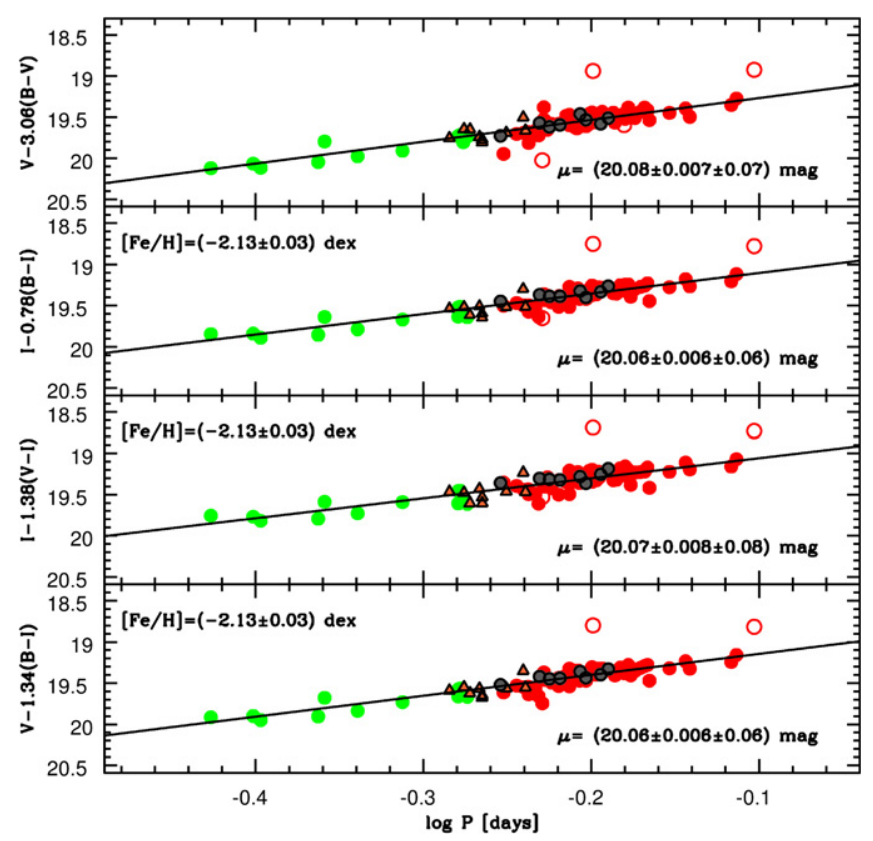

Figure 11. Top: predicted PW $(B V)$ relation (black line). The true distance modulus, the standard error of the mean and the standard deviations are labeled. Note that this PW relation is independent of the metal content. Middle: same as the top, but for the PW $(B I)$ relation. The true distance modulus was estimated using the labeled value of iron abundance. Bottom: same as the middle, but for the PW $(V I)$ relation.

true Carina distance based on empirical calibrators. Moreover, the new distance determination also agrees with Carina distances available in the literature that are based on robust standard candles (see Table 11).

To take advantage of the multiband photometry for Carina RRLs, we estimated the distance using the PWZ $(B I)$, the PWZ $(V I)$, and the triple band PWZ (BIV) relations (see Figure 11). The current pulsation predictions suggest a mild dependence on the metal content. Indeed, the coefficients of the metallicity term are $0.106(B I), 0.150(V I)$, and $0.075(B V I)$. In passing we 


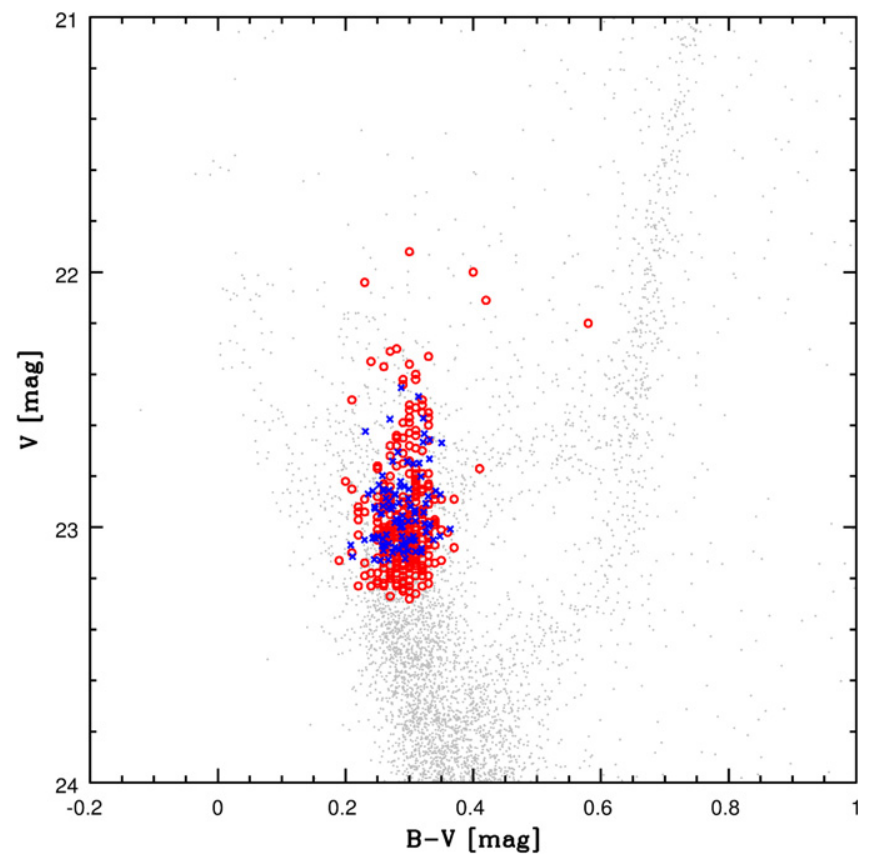

Figure 12. Position of $\mathrm{SX}$ Phe in $V,(B-V)$ CMD. Red circles and blue crosses display the VM13 and the new discovered variables, respectively. Their positions, periods, and magnitudes are given in Tables 13 and 12 .

note that the metallicity dependence of the optical PW relation for RRL stars displays a different trend when compared with similar relations for classical Cepheids. For the latter objects the PW $(V I)$ relation is almost independent of the metallicity, while the PW $(B V)$ shows a strong dependence on the iron content. The reader interested in a detailed discussion of the difference between RRLs and classical Cepheids is referred to the recent paper by M2015.

To estimate the distance from the PWZ $(B I)$, the PWZ $(V I)$ and the PWZ $(B V I)$ we adopted a mean iron abundance for Carina RRLs of $(-2.13 \pm 0.03 \pm 0.28)$ dex (Fabrizio et al. 2015). Using this value we found the following true distance moduli: $\mu=(20.086 \pm 0.006 \pm 0.06) \mathrm{mag}(B I), \quad \mu=$ $(20.07 \pm 0.008 \pm 0.08) \mathrm{mag}(V I)$, and $\mu=(20.06 \pm 0.006 \pm$ $0.06)$ mag $(V B I)$. Note that the uncertainties in the distance modulus account for the photometric errors, the standard deviation of the theoretical PWZ relations and the intrinsic spread in iron abundance. Once again the above distance determinations agree quite well with the empirical distance, with the distance based on the PW $(B V)$, and with distances available in the literature.

\section{SX PHOENICIS}

More that 30 years ago Niss (1981) identified the first variable Blue Straggler (BS) in the Galactic globular $\omega$ Cen. The oscillating BSs have been the cross-road of several theoretical (Gilliland et al. 1998; Santolamazza et al. 2001; Fiorentino et al. 2015b) and observational (Kaluzny \& Thompson 2003; Olech et al. 2005; Fiorentino et al. 2014) investigations. However, no general consensus has been reached yet on their evolutionary and pulsation properties. The names suggested in the literature range from SX Phoenicis (Nemec \& Mateo 1990, p. 134) to Dwarf Cepheids (Mateo et al. 1998; Vivas \& Mateo 2013) to ultra-short-period Cepheids (Eggen 1979) to AI Velorum stars (Bessell 1969) to high-amplitude $\delta$ Scuti stars (McNamara 1995; Hog \& Petersen 1997).

The most relevant point concerning the classification is that SX Phe are considered the metal-poor extension of the classical $\delta$ Scuti stars. Ironically, the prototype, SX Phe itself, is a metalintermediate $([\mathrm{Fe} / \mathrm{H}]=-1.3 \mathrm{dex} ; \mathrm{Hog} \&$ Petersen 1997). The circumstantial empirical evidence concerning the pulsation properties are: they oscillate both in radial and non radial modes and a significant fraction are mixed-mode variables (Gilliland et al. 1998); their amplitude ranges from a few hundredths to a few tenths of magnitude; they do not show a clear separation in the Bailey diagram (luminosity amplitude versus pulsation period), this means that the mode identification based on their pulsation properties is not trivial; they obey a period-luminosity (McNamara 2000) and a period-luminosity-color-metallicity (Petersen \& Christensen-Dalsgaard 1999; Fiorentino et al. 2013).

The region of the CMD in which these objects are located is strongly affected by degeneracy. They are at the transition between low- and intermediate-mass stars during either central hydrogen burning or thick shell hydrogen burning. However, the same region is also crossed by stellar structures approaching the main sequence (Marconi et al. 2000) and by stellar structures that experienced either a collisional merging or a binary merging, i.e., the so-called BSs (Dalessandro et al. 2013).

The above evidence indicates that the evolutionary channel producing field $\delta$ Scuti and SX Phe can hardly be constrained by their position in the CMD or the Bailey diagram. The empirical scenario becomes easier for variables hosted either in open or in globular clusters, since they are typically brighter and bluer than MS turn-off stars. This further supports their "peculiar" evolutionary origin.

In this context, dwarf galaxies play a crucial role. The stellar content of dwarf spheroidal galaxies that experienced only a single star formation event (Cetus Monelli et al. 2012a) and (Tucana Monelli et al. 2010a) appears similar to globular clusters. These stellar systems are dominated by old $(t>10 \mathrm{Gyr})$ stellar populations, therefore the colors of their main sequence turn off stars (MSTO) are systematically redder (cooler) than the red edge of the IS. This means that the Cepheid IS in these stellar systems can only be crossed by BSs, since these objects are hotter and brighter than canonical MSTO stars. However, dwarf galaxies that underwent multiple star formation episodes, in particular a well defined star formation episode 6-9 Gyrs ago, are going to have both "canonical" and "peculiar" objects crossing the Cepheid IS. The Carina dwarf spheroidal galaxy belongs to the latter group.

The above scenario has been soundly supported by the recent detailed photometric investigation by VM13. They identified more than 340 new SX Phe in Carina with periods ranging from 0.03860 to 0.18058 days and luminosity amplitudes ranging from 0.22 to $1.10 \mathrm{mag}$ in the $V$ band. In this context it is worth mentioning that their magnitude $(21.89 \leqslant V \leqslant 23.55)$ and color distribution $(0.05 \leqslant B-V \leqslant 0.54)$ indicate that only a minor fraction belongs to the so-called Blue Plume $(22.0 \leqslant V$ $\leqslant 23.2 ; 0.02 \leqslant B-V \leqslant 0.22)$, i.e., to the objects for which it is not clear whether they are truly young $(t<1 \mathrm{Gyr})$ or BSs of the old populations (Okamoto et al. 2008; Monelli et al. 2012b). The bulk seem to belong to canonical main sequence intermediate-age stars. This evidence opens a new path in the investigation of these interesting objects, since we are dealing 
with objects that are the aftermath of the different channels located at the same distance and covering a narrow range in metal abundances (Fabrizio et al. 2015).

Although, the investigation by VM13 is a substantial step forward in the identification of these objects we decided to further investigate the possible occurrence of SX Phe stars. The working hypothesis was mainly supported by the slope of the Cepheid IS suggesting that the actual sample of SX Phe might be even larger. The similarity of the Cepheid IS with the location of SX Phe, $\delta$ Scuti, and RR Lyrae stars is supported by detailed investigations concerning their pulsation properties (McNamara 2011; McNamara \& Barnes 2014). Thanks to the photometric precision and accuracy of our multiband photometric catalog we identified 101 new SX Phe. Some examples of light curves are plotted in Figure set 3, while their positions, epochs, periods and mean magnitudes are listed in Table 12. Finally, Figure 12 shows the position of VM13 (red circles) and the new discovered (blue crosses) SX Phe in $I V$ (B-V) CMD. The mean magnitudes were estimated as intensity means using an analytical fit of the light curves. Moreover, we confirm the variability for 324 out of the 340 known SX Phe in Carina. For the other 16, we have insufficient data for DC-1, DC-2, DC-3, DC-4, DC-5, DC-6, DC-284, and DC-339, and we do not confirm the variability for DC-75, DC-158, DC-264, and DC295, we do not find DC-180 and DC-340 and we consider DC1, DC-111 and DC-144 the same variable. The pulsation properties for these objects are given in Table 13.

The comparison between predicted and observed evolutionary and pulsation properties of Carina SX Phe will be discussed in a forthcoming paper.

\section{CONCLUSIONS AND FUTURE PERSPECTIVES}

We have discussed new and accurate multiband optical $(U B V I)$ photometry of helium burning variables in the Carina dwarf spheroidal galaxy. The current photometry covers a time interval of more than 20 years. This means that we can provide robust identification of regular variables with periods close to a half and one day, as well as variables showing modulations in the pulsation period and/or in in amplitude (mixed mode pulsators, Blazhko).

We ended up with a sample of 92 RRLs, among them 12 first overtones $\left(\mathrm{RR}_{c}\right), 63$ fundamental $\left(\mathrm{RR}_{a b}\right)$ pulsators, 9 mixedmode variables $\left(\mathrm{RR}_{d}\right)$, and 8 candidate Blazhko variables $(\mathrm{Bl})$. Six out of the 92 RRL variables are new identifications. Moreover, we identified one new double mode pulsator. We also identified $20 \mathrm{ACs}$, and among them 1 is a new identification. Together with all these variables we found two new LPVs and seven EB candidates.

For the entire sample of variables we provide accurate pulsation properties (periods, luminosity amplitudes) plus accurate estimates of the mean $B V I$ magnitudes. The mean $B V$ magnitudes are based on a spline fit, while the mean $I$-band mean magnitude is based on a template fit. For the $\mathrm{RR}_{d}$ variables we have been able to estimate the two oscillating frequencies and also the luminosity amplitude of both the FO and the $\mathrm{F}$ component. The current data do not allow us to constrain the secondary oscillation of the candidate Blazhko RRLs. According to extensive photometric surveys of field RRLs, they are a minor fraction (8\%) of Blazhko RRLs (Soszyński et al. 2011).

Although the pulsation properties of ACs in Carina are very accurate we are still facing the problem of mode identification.
It seems that optical bands do not help us in settling this longstanding problem.

The analysis of the Bailey diagram confirms that Carina is an Oosterhoff intermediate system and shows that the luminosity amplitudes of the $\mathrm{FO}$ component in $\mathrm{RR}_{d}$ variables are located along the long-period tail of "regular" $\mathrm{RR}_{c}$ variables, while the fundamental components are located along the short-period tale of "regular" $\mathrm{RR}_{a b}$ variables. To our knowledge this is the first time that we can properly locate the two components of $\mathrm{RR}_{d}$ variables.

The comparison between theory and observations in the Petersen diagram for $\mathrm{RR}_{d}$ variables indicates that a steady increase in the metal content causes a steady decrease in the period ratio and in the fundamental period. This evidence is supported not only by $\mathrm{RR}_{d}$ variables in Galactic globulars, but also by $\mathrm{RR}_{d}$ variables in the Galactic halo and bulge. Moreover, the same diagram shows that $\mathrm{RR}_{d}$ variables in nearby dwarf spheroidals and dwarf irregulars (the Magellanic Clouds) display similar properties and that Carina $\mathrm{RR}_{d}$ variables are located in a region in which there are only $\mathrm{RR}_{d}$ variables hosted in metal-poor globular clusters (M15, M68), in the Halo, or in metal-poor dwarf spheroidal galaxies (e.g., Draco).

The new accurate and precise mean magnitudes allowed us to provide new independent estimates of Carina's true distance modulus. We investigated four different reddening-free PeriodWesenheit relations $(B V, B I, V I, B V I)$. We found that the $\mathrm{PW}$ $(B V)$ is independent of the metal content. This finding soundly supports recent pulsation predictions based on nonlinear, convective, hydrodynamical models of RRL stars (M2015).

We took advantage of the trigonometric parallaxes for five field RRLs provided by Benedict et al. (2011) to give a new independent estimate of Carina's true distance modulus using the observed slope of the PW $(B V)$ relation. We found $\mu=20.02 \pm 0.02$ (standard error of the mean) \pm 0.05 (standard deviation) mag. The distance was evaluated using the entire sample of variables. In particular, the $\mathrm{RR}_{c}$ variables were fundamentalized. The above estimate agrees, within the errors, with Carina distances available in the literature that are based on solid standard candles (see Table 11).

To take advantage of the new predicted optical and NIR PW relations provided by M2015 we also estimated the Carina distances using the zero-point and the slope of the PWZ $(B V$, $B I, V I, B V I)$ relations. We found true distance moduli of $\mu=(20.08 \pm 0.007 \pm 0.07), \mu=(20.06 \pm 0.006 \pm 0.06)$, $\mu=(20.07 \pm 0.008 \pm 0.08) \mathrm{mag}$, and $\mu=(20.06 \pm 0.006 \pm$ $0.06)$ mag. Note that the distances based on both predicted and empirical PW $(B V)$ relations are independent of the metal content. The distances based on the PWZ ( $B I, V I, B V I)$ relations have been estimated by assuming a mean iron abundance for Carina RRLs of $[\mathrm{Fe} / \mathrm{H}]=(-2.13 \pm 0.03 \pm 0.28)$ dex. All the above distances are independent of uncertainties in the reddening. However, they rely on the assumption that the reddening law adopted to estimate the color coefficients of the PW relations is appropriate. The true distances based on empirical and predicted PW relations agree quite well with each other and with similar distances available in the literature.

There is evidence that distances based on the theoretical calibrations are $\sim 0.05-0.1 \mathrm{mag}$ larger than the distance based on empirical calibrations. The evidence applies not only to the PW relation that is independent of metallicity, but also to the PW relation based on triple bands indicates that the 
Table 12

Pulsation Properties of the New Carina SX Phe

\begin{tabular}{|c|c|c|c|c|c|c|}
\hline ID & $\alpha$ & $\delta$ & Period (day) & $\langle B\rangle^{b}$ & $\langle V\rangle^{b}$ & $\langle I\rangle^{b}$ \\
\hline 341 & 064014.14 & -510123.4 & 0.05898004 & 23.11 & 22.85 & 22.42 \\
\hline 342 & 064015.46 & -510831.2 & 0.06404925 & 23.25 & 22.94 & 22.50 \\
\hline 343 & 064017.76 & -505606.0 & 0.06189917 & 22.99 & 22.70 & 22.33 \\
\hline 344 & 064023.45 & -510018.5 & 0.05700707 & 23.39 & 23.04 & 22.49 \\
\hline 345 & 064023.84 & -505516.0 & 0.0616327 & 23.28 & 23.04 & 22.65 \\
\hline 346 & 064026.44 & -505512.2 & 0.06983306 & 23.20 & 22.93 & 22.48 \\
\hline 347 & 064034.03 & -505636.9 & 0.06382168 & 23.17 & 22.92 & 22.49 \\
\hline 348 & 064038.49 & -505034.2 & 0.05887662 & 23.01 & 22.74 & 22.34 \\
\hline 349 & 064038.82 & -511352.6 & 0.05220283 & 23.32 & 23.03 & 22.61 \\
\hline 350 & 064040.71 & -505829.0 & 0.05225971 & 23.12 & 22.86 & 22.49 \\
\hline 351 & 064049.07 & -505310.6 & 0.06510992 & 23.34 & 23.04 & 22.62 \\
\hline 352 & 064049.13 & -505437.1 & 0.05555380 & 22.84 & 22.58 & 22.16 \\
\hline 353 & 064049.50 & $\begin{array}{lll}-51 & 0126.7\end{array}$ & 0.05860522 & 23.17 & 22.92 & 22.62 \\
\hline 354 & 064055.07 & -505518.3 & 0.0559396 & 23.35 & 23.06 & 22.65 \\
\hline 355 & 064058.61 & -505505.4 & 0.06195699 & 23.33 & 23.07 & 22.74 \\
\hline 356 & 064059.22 & -505652.2 & 0.05387742 & 23.26 & 22.97 & 22.55 \\
\hline 357 & 064102.69 & -505705.9 & 0.05802369 & 23.41 & 23.09 & 22.66 \\
\hline 358 & 064103.13 & -505151.1 & 0.04853505 & 23.10 & 22.86 & 22.51 \\
\hline 359 & 064102.86 & -505800.1 & 0.05870867 & 23.29 & 23.04 & 22.49 \\
\hline 360 & 064104.51 & -505507.3 & 0.05059894 & 23.05 & 22.80 & 22.41 \\
\hline 361 & 064107.09 & -504750.9 & 0.05405631 & 23.33 & 23.07 & 22.59 \\
\hline 362 & 064106.85 & $\begin{array}{lll}-51 & 04 & 14.1\end{array}$ & 0.05004092 & 22.89 & 22.57 & 22.18 \\
\hline 363 & 064108.60 & -505435.8 & 0.0591939 & 23.29 & 23.04 & 22.67 \\
\hline 364 & 064111.16 & -510011.8 & 0.06534271 & 23.29 & 23.00 & 22.63 \\
\hline 365 & 064112.44 & -504531.2 & 0.05781447 & 23.33 & 23.06 & 22.60 \\
\hline 366 & 064113.10 & $\begin{array}{lll}-51 & 09 & 07.4\end{array}$ & 0.05277468 & 23.28 & 23.04 & 22.64 \\
\hline 367 & $0641 \quad 14.32$ & -510157.8 & 0.06230673 & 23.19 & 22.90 & 22.45 \\
\hline 368 & $0641 \quad 14.88$ & -505516.6 & 0.07088727 & 23.21 & 22.88 & 22.46 \\
\hline 369 & 064115.33 & -505512.5 & 0.05923093 & 23.29 & 23.03 & 22.63 \\
\hline 370 & 064116.36 & -505436.0 & 0.05263070 & 23.24 & 22.97 & 22.53 \\
\hline 371 & $0641 \quad 18.51$ & -505148.0 & 0.0610961 & 23.14 & 22.87 & 22.49 \\
\hline 372 & 064123.09 & -510742.1 & 0.06317447 & 22.99 & 22.66 & 22.28 \\
\hline 373 & 064124.30 & -505925.6 & 0.05712586 & 23.41 & 23.10 & 22.61 \\
\hline 374 & 064124.29 & -510421.8 & 0.05256096 & 23.02 & 22.67 & 22.28 \\
\hline 375 & 064125.07 & -505553.9 & 0.07412219 & 23.06 & 22.75 & 22.33 \\
\hline 376 & 064126.05 & -511219.0 & 0.0739414 & 23.20 & 22.86 & 22.46 \\
\hline 377 & 064128.18 & -505526.9 & 0.05301314 & 23.06 & 22.73 & 22.34 \\
\hline 378 & 064128.80 & -504311.2 & 0.05483068 & 23.42 & 23.12 & 22.71 \\
\hline 379 & 064128.81 & -510020.0 & 0.07092323 & 23.12 & 22.80 & 22.38 \\
\hline 380 & 064129.87 & -505205.1 & 0.06527955 & 22.99 & 22.67 & 22.27 \\
\hline 381 & 064132.44 & -504757.3 & 0.07488229 & 22.96 & 22.63 & 22.19 \\
\hline 382 & 064132.63 & -505743.7 & 0.07197212 & 23.31 & 22.98 & 22.63 \\
\hline 383 & 064132.93 & -505254.2 & 0.1594306 & 23.28 & 23.07 & 22.78 \\
\hline 384 & 064133.43 & -505248.5 & 0.05863985 & 23.32 & 22.98 & 22.49 \\
\hline 385 & 064133.65 & -505737.8 & 0.05633247 & 22.80 & 22.49 & 22.19 \\
\hline 386 & 064135.77 & -510039.1 & 0.0636862 & 23.20 & 22.95 & 22.52 \\
\hline 387 & 064136.96 & -505747.0 & 0.05627784 & 23.37 & 23.13 & 22.73 \\
\hline 388 & 064137.74 & -505513.6 & 0.06400101 & 23.15 & 22.85 & 22.52 \\
\hline 389 & 064137.61 & -505858.0 & 0.07160168 & 23.06 & 22.75 & 22.44 \\
\hline 390 & 064139.58 & -505701.2 & 0.0582159 & 23.32 & 22.99 & 22.60 \\
\hline 391 & 064142.92 & -505231.4 & 0.06104802 & 23.23 & 22.91 & 22.43 \\
\hline 392 & 064144.17 & -504422.9 & 0.06385933 & 22.74 & 22.45 & 21.87 \\
\hline 393 & 064144.84 & -510348.3 & 0.05746218 & 23.37 & 23.09 & 22.72 \\
\hline 394 & 064145.69 & -505001.3 & 0.04855492 & 23.15 & 22.89 & 22.62 \\
\hline 395 & 064149.86 & -510021.5 & 0.05655043 & 22.86 & 22.62 & 22.33 \\
\hline 396 & 064149.87 & -510032.4 & 0.06046970 & 23.26 & 22.94 & 22.55 \\
\hline 397 & 064151.00 & -505805.9 & 0.0645517 & 23.40 & 23.09 & 22.70 \\
\hline 398 & 064151.59 & -505305.0 & 0.06014112 & 23.39 & 23.09 & 22.67 \\
\hline 399 & 064151.66 & -505608.9 & 0.04527754 & 23.17 & 22.93 & 22.58 \\
\hline 400 & 064157.09 & -505508.0 & 0.06364862 & 23.24 & 22.95 & 22.49 \\
\hline 401 & 064158.08 & -505758.1 & 0.05734282 & 23.12 & 22.84 & 22.47 \\
\hline 402 & 064159.28 & -510000.0 & 0.07441239 & 23.12 & 22.85 & 22.39 \\
\hline 403 & 064159.76 & -505535.4 & 0.04671546 & 23.17 & 22.90 & 22.48 \\
\hline 404 & 064200.27 & -505219.9 & 0.06205412 & 23.28 & 22.97 & 22.63 \\
\hline
\end{tabular}


Table 12

(Continued)

\begin{tabular}{|c|c|c|c|c|c|c|}
\hline ID & $\alpha$ & $\delta$ & Period (day) & $\langle B\rangle^{b}$ & $\langle V\rangle^{b}$ & $\langle I\rangle^{b}$ \\
\hline 405 & 064201.47 & -505227.1 & 0.06348319 & 23.37 & 23.01 & 22.55 \\
\hline 406 & 064201.82 & -505130.4 & 0.06373945 & 23.04 & 22.74 & 22.35 \\
\hline 407 & 064203.52 & -505733.5 & 0.05525245 & 23.19 & 22.92 & 23.19 \\
\hline 408 & 064203.49 & $\begin{array}{lll}-51 & 01 & 12.2\end{array}$ & 0.05567260 & 23.20 & 22.95 & 22.54 \\
\hline 409 & 064205.51 & -505743.0 & 0.05779336 & 23.38 & 23.03 & 22.66 \\
\hline 410 & 064207.77 & -510051.5 & 0.05261781 & 23.19 & 22.92 & 22.45 \\
\hline 411 & 064208.13 & -505236.7 & 0.06459468 & 23.11 & 22.82 & 22.37 \\
\hline 412 & 064209.22 & -505536.2 & 0.05646218 & 23.27 & 22.98 & 22.58 \\
\hline 413 & 064209.61 & $\begin{array}{lll}-51 & 0103.4\end{array}$ & 0.06032828 & 23.34 & 23.02 & 22.54 \\
\hline 414 & $0642 \quad 12.67$ & -505623.8 & 0.05842271 & 23.22 & 22.87 & 22.42 \\
\hline 415 & $0642 \quad 13.13$ & -505601.6 & 0.05598982 & 23.18 & 22.89 & 22.44 \\
\hline 416 & $0642 \quad 17.19$ & -505118.2 & 0.05422022 & 23.33 & 23.07 & 22.63 \\
\hline 417 & $\begin{array}{lll}06 & 42 & 17.68\end{array}$ & -504839.2 & 0.06434278 & 23.26 & 22.98 & 22.63 \\
\hline 418 & 064219.08 & -504746.7 & 0.05607941 & 23.22 & 22.92 & 22.50 \\
\hline 419 & 064219.88 & -504504.8 & 0.05780775 & 23.36 & 23.07 & 22.50 \\
\hline 420 & 064221.25 & -504535.8 & 0.06575657 & 23.15 & 22.87 & 22.43 \\
\hline 421 & 064222.01 & -505006.5 & 0.05161476 & 23.36 & 23.07 & 22.65 \\
\hline 422 & $0642 \quad 23.24$ & -505450.4 & 0.07029254 & 23.13 & 22.84 & 22.23 \\
\hline 423 & 064223.67 & -504916.7 & 0.06527164 & 23.08 & 22.83 & 22.48 \\
\hline 424 & $0642 \quad 24.87$ & -510302.1 & 0.07014507 & 23.22 & 22.92 & 22.37 \\
\hline 425 & 064225.71 & -505700.4 & 0.05172937 & 23.28 & 23.00 & 22.53 \\
\hline 426 & 064230.36 & -504905.9 & 0.06320298 & 23.10 & 22.87 & 22.50 \\
\hline 427 & 064231.01 & -504602.6 & 0.05587489 & 23.38 & 23.13 & 22.70 \\
\hline 428 & 064231.58 & -505728.1 & 0.05228718 & 23.36 & 23.05 & 22.65 \\
\hline 429 & 064233.88 & -504846.0 & 0.05360831 & 23.33 & 23.11 & 22.53 \\
\hline 430 & 064233.99 & -504803.3 & 0.05866563 & 23.39 & 23.10 & 22.64 \\
\hline 431 & 064235.21 & -505504.0 & 0.05962675 & 23.40 & 23.08 & 22.66 \\
\hline 432 & 064235.53 & -510222.6 & 0.05995828 & 23.35 & 23.05 & 22.58 \\
\hline 433 & 064237.57 & -505741.4 & 0.05497264 & 23.37 & 23.10 & 22.67 \\
\hline 434 & 064238.39 & -505316.7 & 0.07192960 & 23.24 & 22.93 & 22.43 \\
\hline 435 & 064241.09 & -504938.2 & 0.06649085 & 23.34 & 23.04 & 22.64 \\
\hline 436 & 064242.07 & -505731.2 & 0.05926036 & 23.39 & 23.13 & 22.70 \\
\hline 437 & 064249.56 & -505153.9 & 0.05158018 & 23.30 & 23.05 & 22.70 \\
\hline 438 & 064255.43 & -505443.3 & 0.06550635 & 23.29 & 23.00 & 22.60 \\
\hline 439 & 064255.57 & -505255.2 & 0.06189711 & 23.35 & 23.08 & 22.59 \\
\hline 440 & 064301.04 & -505207.7 & 0.05804751 & 23.35 & 23.09 & 22.70 \\
\hline 441 & 064304.87 & -505345.1 & 0.06346540 & 23.26 & 22.98 & 22.62 \\
\hline 442 & 064325.38 & -505226.9 & 0.05947808 & 23.28 & 23.05 & 22.65 \\
\hline
\end{tabular}

difference is mainly caused by a difference in the zero-point. The comparison between distances based on empirical and on predicted PW relations for stellar systems with precise distances are required to constrain possible systematics either in the current trigonometric parallaxes or in the predicted luminosities at fixed mass.

The above findings further support the mature phase that RRLs are approaching as distance indicators. This circumstantial evidence is going to be reinforced using either NIR and/or MIR PW relations (Madore et al. 2013; Braga et al. 2015). The key advantage of the above findings is that RRL PW relations display either a mild or a vanishing metallicity dependence. This offers a unique opportunity to estimate individual distances for a significant fraction of Halo and Bulge RRLs that have been recently discovered by long-term photometric surveys (OGLE IV, Soszyński et al. 2014; Catalina, Drake et al. 2009; LINEAR, Palaversa et al. 2013; LONEOS, Miceli et al. 2008; ASAS, Pojmanski 2002; QUEST, Vivas et al. 2004; Gaia, Eyer et al. 2012). This appears to be a crucial stepping stone before RRLs can be used as solid primary distance indicators in the local universe.

The recent findings concerning the difference in iron and in $\mathrm{Mg}$ distribution between the old and the intermediate-age
Carina stellar populations opened the path for a more detailed pulsation analysis of Carina SX Phe stars. In a recent investigation Fiorentino et al. (2015b) found that the pulsation properties of SX Phe can provide solid constraints on their pulsation masses. This approach has already been applied to canonical Galactic globulars (NGC 6541, Fiorentino et al. 2014) and to Omega Centauri, i.e., in stellar systems mainly hosting old stellar populations. The sizable sample of Carina SX Phe stars is a very promising laboratory to constrain the mass distribution of the different stellar populations. Moreover, they are located at the same distance, and therefore they offer a unique opportunity to constrain whether the aftermaths of single and binary star evolution display different massluminosity relations. This means the prodrome for their use as solid distance indicators.

It is a real pleasure to thank the referee, Mario Mateo, for the positive opinion concerning the content of the paper and for several suggestions and insights that improved the readability and the cut of the paper. This work was partially supported by PRININAF 2011 "Tracing the formation and evolution of the Galactic halo with VST" (PI: M. Marconi) and by PRIN-MIUR (2010LY5N2T) "Chemical and dynamical evolution of the Milky Way and Local Group galaxies" (PI: F. Matteucci). M. 
Table 13

Pulsation Properties of the Known Carina SX Phe

\begin{tabular}{|c|c|c|c|c|c|c|c|}
\hline ID & $\alpha$ & $\delta$ & Period (day) & $\langle B\rangle^{b}$ & $\langle V\rangle^{b}$ & $\langle I\rangle^{b}$ & M98 \\
\hline 7 & 063953.11 & -510431.5 & 0.05861265 & 23.43 & 23.13 & 22.12 & $\ldots$ \\
\hline 8 & 063956.16 & -510643.6 & 0.0840233 & 22.66 & 22.36 & 21.92 & $\ldots$ \\
\hline 9 & 064000.79 & -505758.5 & 0.06283058 & 23.33 & 23.04 & 22.45 & $\ldots$ \\
\hline 10 & 064003.36 & -510755.6 & 0.06600411 & 23.19 & 22.93 & 22.43 & $\ldots$ \\
\hline 11 & 064006.08 & -505558.0 & 0.0566776 & 23.32 & 23.04 & 22.58 & $\ldots$ \\
\hline 12 & 064014.68 & -510505.0 & 0.05116040 & 23.46 & 23.18 & $\ldots$ & $\ldots$ \\
\hline 13 & 064017.93 & -505633.7 & 0.0572522 & 23.27 & 23.01 & 22.59 & $\ldots$ \\
\hline 14 & 064018.59 & $\begin{array}{lll}-51 & 0301.9\end{array}$ & 0.05801669 & 23.33 & 23.06 & 22.67 & $\ldots$ \\
\hline 15 & 064021.39 & $\begin{array}{llll}-51 & 01 & 02.2\end{array}$ & 0.06133516 & 23.14 & 22.92 & 22.57 & $\ldots$ \\
\hline 16 & 064023.91 & -510644.4 & 0.06490812 & 23.16 & 22.90 & 22.50 & $\ldots$ \\
\hline 17 & 064027.16 & -510617.6 & 0.06391992 & 23.31 & 22.99 & 22.52 & $\ldots$ \\
\hline 18 & 064027.18 & $\begin{array}{lll}-51 & 01 & 03.4\end{array}$ & 0.06005208 & 23.36 & 23.07 & 22.66 & $\ldots$ \\
\hline 19 & 064028.33 & -505258.8 & 0.05846323 & 23.16 & 22.94 & 22.64 & $\ldots$ \\
\hline 20 & 064029.07 & -505558.4 & 0.05692126 & 23.28 & 23.01 & 22.49 & $\ldots$ \\
\hline 21 & 064031.67 & -510854.2 & 0.07130197 & 23.12 & 22.81 & 22.37 & $\ldots$ \\
\hline 22 & 064032.86 & -510143.4 & 0.05866617 & 23.27 & 23.00 & 22.54 & $\ldots$ \\
\hline 23 & 064035.66 & -505648.1 & 0.05035238 & 23.51 & 23.23 & 22.83 & $\ldots$ \\
\hline 24 & 064037.79 & -505811.5 & 0.05697949 & 23.30 & 23.03 & 22.61 & $\ldots$ \\
\hline 25 & 064038.21 & -505804.9 & 0.05765942 & 23.31 & 23.03 & 22.58 & $\ldots$ \\
\hline 26 & 064038.84 & -505839.7 & 0.05955433 & 23.28 & 23.03 & 22.64 & $\ldots$ \\
\hline 27 & 064039.93 & -510032.7 & 0.06096031 & 23.28 & 22.99 & 22.61 & $\ldots$ \\
\hline 28 & 064040.56 & -510230.7 & 0.06020401 & 23.08 & 22.77 & 22.35 & $\ldots$ \\
\hline 29 & 064041.70 & -505910.5 & 0.05311877 & 23.11 & 22.84 & 22.44 & $\ldots$ \\
\hline 30 & 064045.02 & -505929.4 & 0.06503761 & 23.02 & 22.70 & 22.30 & $\ldots$ \\
\hline 31 & 064045.26 & -510618.6 & 0.06037914 & 23.35 & 23.04 & 22.56 & $\ldots$ \\
\hline 32 & 064046.39 & -505753.4 & 0.05804718 & 23.26 & 22.98 & 22.58 & $\ldots$ \\
\hline 33 & 064046.64 & -510937.3 & 0.05529873 & 23.38 & 23.09 & 22.65 & $\ldots$ \\
\hline 34 & 064046.76 & -505217.5 & 0.05526398 & 23.29 & 23.03 & 22.64 & $\ldots$ \\
\hline 35 & 064047.68 & -505644.1 & 0.06166175 & 23.26 & 22.94 & 22.48 & $\ldots$ \\
\hline 36 & 064048.00 & -505833.9 & 0.05985196 & 23.17 & 22.90 & 22.49 & $\ldots$ \\
\hline 37 & 064048.47 & -511055.6 & 0.05410886 & 23.36 & 23.03 & 22.62 & $\ldots$ \\
\hline 38 & 064048.81 & -510041.2 & 0.05365485 & 23.32 & 23.02 & 22.58 & $\ldots$ \\
\hline 39 & 064050.04 & -505924.6 & 0.05440634 & 23.28 & 23.01 & 22.57 & $\ldots$ \\
\hline 40 & 064050.29 & -510931.2 & 0.05952932 & 23.24 & 22.91 & 22.47 & $\ldots$ \\
\hline 41 & 064050.86 & -505928.7 & 0.06489616 & 22.82 & 22.50 & 22.11 & $\ldots$ \\
\hline 42 & 064051.77 & -510716.9 & 0.05697602 & 23.18 & 22.87 & 22.48 & $\ldots$ \\
\hline 43 & 064053.52 & -510714.6 & 0.06162396 & 23.25 & 22.94 & 22.46 & $\ldots$ \\
\hline 44 & 064054.40 & $\begin{array}{lll}-51 & 0128.4\end{array}$ & 0.06879427 & 22.27 & 22.04 & 21.49 & $\ldots$ \\
\hline 45 & 064055.63 & -510043.0 & 0.05688051 & 23.39 & 23.09 & 22.72 & V4 \\
\hline 46 & 064057.65 & -505609.5 & 0.06778138 & 23.04 & 22.74 & 22.26 & $\ldots$ \\
\hline 47 & 064057.88 & $\begin{array}{lll}-51 & 0207.9\end{array}$ & 0.05986604 & 23.23 & 22.98 & 22.48 & $\ldots$ \\
\hline 48 & 064059.18 & -504944.3 & 0.0565391 & 23.31 & 23.04 & 22.74 & $\ldots$ \\
\hline 49 & 064059.27 & -505031.6 & 0.05626531 & 23.40 & 23.14 & 22.69 & $\ldots$ \\
\hline 50 & 064059.31 & -505649.1 & 0.07320810 & 22.71 & 22.42 & 22.00 & $\ldots$ \\
\hline 51 & 064059.92 & -504844.6 & 0.05879641 & 23.37 & 23.08 & 22.50 & $\ldots$ \\
\hline 52 & 064100.18 & -510726.7 & 0.06380392 & 23.31 & 23.00 & 22.55 & $\ldots$ \\
\hline 53 & 064100.50 & -505453.6 & 0.05843550 & 23.27 & 22.99 & 22.51 & $\ldots$ \\
\hline 54 & 064100.89 & -510001.1 & 0.06134829 & 23.33 & 23.06 & 22.64 & $\ldots$ \\
\hline 55 & 064102.48 & -510025.8 & 0.05870253 & 23.32 & 23.01 & 22.65 & V5 \\
\hline 56 & 064102.98 & -505841.1 & 0.05851668 & 23.29 & 23.00 & 22.59 & V12 \\
\hline 57 & 064103.29 & -505614.8 & 0.05586523 & 23.31 & 23.01 & 22.15 & $\ldots$ \\
\hline 58 & 064103.89 & -505844.1 & 0.05123522 & 23.54 & 23.27 & 22.59 & $\ldots$ \\
\hline 59 & 064105.03 & $\begin{array}{lll}-51 & 05 & 15.2\end{array}$ & 0.06586275 & 22.88 & 22.59 & 22.18 & $\ldots$ \\
\hline 60 & 064105.44 & $-5048 \quad 27.0$ & 0.05964722 & 23.32 & 23.04 & 22.42 & $\ldots$ \\
\hline 61 & 064105.72 & -505744.1 & 0.06627047 & 22.84 & 22.54 & 22.13 & $\ldots$ \\
\hline 62 & 064106.14 & -505151.3 & 0.04596514 & 23.45 & 23.23 & $\ldots$ & $\ldots$ \\
\hline 63 & 064106.37 & -504923.2 & 0.05920742 & 23.37 & 23.06 & 22.53 & $\ldots$ \\
\hline 64 & 064106.61 & -510844.1 & 0.05839355 & 23.37 & 23.07 & 22.64 & V10 \\
\hline 65 & 064106.93 & -505918.4 & 0.06264205 & 23.26 & 23.00 & 22.57 & $\ldots$ \\
\hline 66 & 064107.69 & -505234.0 & 0.0525963 & 23.37 & 23.10 & 22.65 & $\ldots$ \\
\hline 67 & 064107.88 & -505600.9 & 0.06022396 & 23.35 & 23.06 & 22.61 & V16 \\
\hline 68 & 064108.76 & -505427.4 & 0.06496999 & 23.15 & 22.84 & 22.31 & $\ldots$ \\
\hline 69 & 064109.70 & -505737.9 & 0.05489874 & 23.33 & 23.01 & 22.61 & $\ldots$ \\
\hline 70 & 064111.27 & -505741.0 & 0.05504275 & 22.94 & 22.65 & 22.22 & $\ldots$ \\
\hline
\end{tabular}


Table 13

(Continued)

\begin{tabular}{|c|c|c|c|c|c|c|c|}
\hline ID & $\alpha$ & $\delta$ & Period (day) & $\langle B\rangle^{b}$ & $\langle V\rangle^{b}$ & $\langle I\rangle^{b}$ & M98 \\
\hline$\overline{71}$ & 064111.73 & -510135.5 & 0.05628829 & 23.36 & 23.06 & 22.69 & $\ldots$ \\
\hline 72 & 064112.00 & -505301.6 & 0.05684917 & 23.48 & 23.18 & 22.68 & $\ldots$ \\
\hline 73 & $0641 \quad 12.06$ & -505640.2 & 0.05332273 & 23.55 & 23.25 & 22.79 & $\ldots$ \\
\hline 74 & $0641 \quad 12.44$ & -505310.8 & 0.06307089 & 22.90 & 22.57 & 22.09 & $\ldots$ \\
\hline 76 & $0641 \quad 13.33$ & -505631.5 & 0.07069207 & 22.84 & 22.53 & 22.06 & $\ldots$ \\
\hline 77 & $0641 \quad 13.43$ & -505308.2 & 0.0641156 & 23.17 & 22.86 & 22.48 & $\ldots$ \\
\hline 78 & $0641 \quad 14.31$ & -505932.8 & 0.07554039 & 23.11 & 22.79 & 22.41 & V9 \\
\hline 79 & 064114.59 & -510249.3 & 0.06153187 & 23.19 & 22.93 & 22.51 & $\ldots$ \\
\hline 80 & $0641 \quad 14.69$ & -510844.1 & 0.05600743 & 23.35 & 23.09 & 22.59 & $\ldots$ \\
\hline 81 & $0641 \quad 14.75$ & -505920.3 & 0.06095969 & 22.53 & 22.11 & 21.50 & $\ldots$ \\
\hline 82 & $0641 \quad 15.38$ & $\begin{array}{lll}-51 & 08 & 24.3\end{array}$ & 0.05954823 & 22.93 & 22.60 & 22.16 & $\ldots$ \\
\hline 83 & $0641 \quad 15.89$ & -505544.0 & 0.05754453 & 23.17 & 22.92 & 22.56 & V15 \\
\hline 84 & $0641 \quad 16.15$ & -510256.8 & 0.06525352 & 23.25 & 22.96 & 22.60 & $\ldots$ \\
\hline 85 & $0641 \quad 16.81$ & -510355.9 & 0.06078077 & 23.26 & 22.89 & 21.68 & $\ldots$ \\
\hline 86 & $0641 \quad 16.87$ & -505305.0 & 0.06751050 & 23.00 & 22.71 & 22.28 & $\ldots$ \\
\hline 87 & $0641 \quad 16.89$ & -510345.9 & 0.06586277 & 22.88 & 22.59 & 22.18 & $\ldots$ \\
\hline 88 & $0641 \quad 17.29$ & -510526.6 & 0.05904829 & 22.87 & 22.55 & 22.06 & $\ldots$ \\
\hline 89 & $0641 \quad 17.81$ & -505601.9 & 0.05871582 & 22.99 & 22.72 & 22.31 & $\ldots$ \\
\hline 90 & $0641 \quad 18.05$ & -505640.5 & 0.06652948 & 22.59 & 22.35 & 22.02 & V14 \\
\hline 91 & $0641 \quad 18.09$ & $\begin{array}{lll}-51 & 03 & 17.0\end{array}$ & 0.06557268 & 23.14 & 22.83 & 22.39 & $\ldots$ \\
\hline 92 & $0641 \quad 18.13$ & -505934.1 & 0.05762093 & 23.55 & 23.23 & 22.80 & $\ldots$ \\
\hline 93 & $0641 \quad 18.2$ & -505252.5 & 0.05359279 & 23.41 & 23.11 & 22.69 & $\ldots$ \\
\hline 94 & $0641 \quad 19.06$ & -505610.9 & 0.0604939 & 23.33 & 23.04 & 22.54 & $\ldots$ \\
\hline 95 & $0641 \quad 19.67$ & -510957.5 & 0.05337312 & 23.33 & 22.99 & 22.52 & $\ldots$ \\
\hline 96 & 064120.01 & -50595.9 & 0.06881335 & 22.98 & 22.68 & 22.29 & $\ldots$ \\
\hline 97 & 064120.42 & -505940.7 & 0.06044260 & 23.24 & 22.89 & 22.45 & $\ldots$ \\
\hline 98 & 064120.9 & -50583.0 & 0.05979826 & 23.32 & 23.04 & 22.48 & $\ldots$ \\
\hline 99 & 064121.19 & -51026.5 & 0.06289669 & 23.26 & 22.99 & 22.53 & $\ldots$ \\
\hline 100 & 064121.42 & -505713.2 & 0.05890563 & 23.43 & 23.13 & 22.79 & $\ldots$ \\
\hline 101 & 064121.56 & -510041.5 & 0.05224501 & 23.42 & 23.12 & 22.77 & $\ldots$ \\
\hline 102 & 064121.92 & -50577.7 & 0.05272663 & 23.31 & 23.04 & 22.70 & $\ldots$ \\
\hline 103 & 064122.15 & -505713.5 & 0.05679868 & 22.87 & 22.57 & 22.19 & $\ldots$ \\
\hline 104 & 064122.26 & -50579.4 & 0.05467434 & 23.17 & 22.90 & 22.48 & $\ldots$ \\
\hline 105 & 064122.36 & -505856.5 & 0.0604251 & 23.33 & 23.07 & 22.71 & $\ldots$ \\
\hline 106 & 064122.62 & -505148.9 & 0.07006015 & 22.84 & 22.53 & 22.07 & $\ldots$ \\
\hline 107 & 064123.17 & -510154.1 & 0.06589533 & 23.21 & 22.91 & 22.49 & $\ldots$ \\
\hline 108 & 064123.31 & -51017.3 & 0.06002635 & 23.39 & 23.12 & 22.69 & $\ldots$ \\
\hline 109 & 064123.56 & -505355.7 & 0.06008917 & 23.36 & 23.05 & 22.60 & $\ldots$ \\
\hline 110 & 064123.56 & -510256.0 & 0.05742446 & 23.43 & 23.14 & 22.76 & $\ldots$ \\
\hline 112 & 064125.16 & -504227.2 & 0.05093029 & 23.02 & 22.77 & 22.23 & $\ldots$ \\
\hline 113 & 064125.22 & -505820.6 & 0.05366079 & 23.37 & 23.10 & 22.57 & $\ldots$ \\
\hline 114 & 064125.47 & $\begin{array}{lll}-51 & 00 & 12.5\end{array}$ & 0.06051002 & 22.58 & 22.31 & 21.93 & $\ldots$ \\
\hline 115 & 064126.0 & $\begin{array}{lll}-51 & 03 & 26.0\end{array}$ & 0.06565715 & 23.38 & 23.10 & 22.63 & $\ldots$ \\
\hline 116 & 064126.02 & -51065.0 & 0.05160868 & 23.48 & 23.16 & 22.83 & $\ldots$ \\
\hline 117 & 064126.17 & $-5107 \quad 16.5$ & 0.05743431 & 23.52 & 23.19 & 22.82 & $\ldots$ \\
\hline 118 & 064126.34 & -505743.9 & 0.05157820 & 23.54 & 23.25 & 22.76 & $\ldots$ \\
\hline 119 & 064126.39 & -505620.9 & 0.05193595 & 23.50 & 23.19 & 22.80 & $\ldots$ \\
\hline 120 & 064126.67 & -505821.6 & 0.06033252 & 23.04 & 22.75 & 22.37 & $\ldots$ \\
\hline 121 & 064127.07 & -50530.9 & 0.05577754 & 23.29 & 23.02 & 22.58 & $\ldots$ \\
\hline 122 & 064127.8 & -510126.0 & 0.04680622 & 23.19 & 22.89 & 22.45 & $\cdots$ \\
\hline 123 & 064127.8 & $\begin{array}{lll}-50 & 59 & 1.0\end{array}$ & 0.05847475 & 23.44 & 23.15 & 22.67 & $\ldots$ \\
\hline 124 & 064127.83 & -505541.8 & 0.05863472 & 23.21 & 22.87 & 22.48 & $\ldots$ \\
\hline 125 & 064128.21 & -505337.1 & 0.05675304 & 23.17 & 22.84 & 22.38 & $\ldots$ \\
\hline 126 & 064128.45 & -50598.5 & 0.06369570 & 23.10 & 22.84 & 22.51 & $\ldots$ \\
\hline 127 & 064128.71 & -505811.1 & 0.0601055 & 23.39 & 23.06 & 22.69 & $\ldots$ \\
\hline 128 & 064128.8 & -505135.4 & 0.0527838 & 23.31 & 23.04 & 22.63 & $\ldots$ \\
\hline 129 & 064129.04 & -505319.0 & 0.0544041 & 23.35 & 23.08 & 22.51 & $\cdots$ \\
\hline 130 & 064129.21 & -510023.5 & 0.05956936 & 22.84 & 22.52 & 22.07 & $\ldots$ \\
\hline 131 & 064129.35 & -505544.1 & 0.06545531 & 23.25 & 22.96 & 22.46 & $\ldots$ \\
\hline 132 & 064129.44 & -505739.1 & 0.06719052 & 23.25 & 22.96 & 22.56 & V13 \\
\hline 133 & 064129.67 & -505314.4 & 0.05464765 & 23.39 & 23.11 & 22.61 & $\ldots$ \\
\hline 134 & 064129.85 & $\begin{array}{llll}-51 & 02 & 34.7\end{array}$ & 0.05504185 & 23.44 & 23.14 & 22.71 & $\ldots$ \\
\hline 135 & 064129.87 & -505937.8 & 0.0534139 & 23.49 & 23.17 & 22.69 & $\ldots$ \\
\hline 136 & 064130.25 & $\begin{array}{lll}-51 & 09 & 3.2\end{array}$ & 0.04970627 & 23.40 & 23.10 & 22.54 & $\ldots$ \\
\hline
\end{tabular}


Table 13

(Continued)

\begin{tabular}{|c|c|c|c|c|c|c|c|}
\hline ID & $\alpha$ & $\delta$ & Period (day) & $\langle B\rangle^{b}$ & $\langle V\rangle^{b}$ & $\langle I\rangle^{b}$ & M98 \\
\hline 137 & 064130.42 & -505129.1 & 0.0529662 & 23.36 & 23.10 & 22.72 & $\ldots$ \\
\hline 138 & 064130.47 & -504815.3 & 0.05815515 & 23.41 & 23.09 & $\ldots$ & $\ldots$ \\
\hline 139 & 064130.79 & -505523.3 & 0.0644954 & 23.26 & 22.94 & 22.52 & $\ldots$ \\
\hline 140 & 064130.97 & -510627.7 & 0.05051116 & 23.45 & 23.08 & $\ldots$ & $\ldots$ \\
\hline 141 & 064132.25 & $\begin{array}{lll}-51 & 02 & 38.5\end{array}$ & 0.05788590 & 23.48 & 23.14 & 22.76 & $\ldots$ \\
\hline 142 & 064132.56 & -510056.0 & 0.06607394 & 23.31 & 22.97 & 22.52 & $\ldots$ \\
\hline 143 & 064132.68 & -505258.7 & 0.06626448 & 23.27 & 22.97 & 22.55 & $\ldots$ \\
\hline 145 & 064132.88 & -505721.9 & 0.05559422 & 23.30 & 23.05 & 22.53 & $\ldots$ \\
\hline 146 & 064133.29 & -505552.0 & 0.05518256 & 23.42 & 23.14 & 22.64 & $\ldots$ \\
\hline 147 & 064133.31 & -505621.0 & 0.0516737 & 23.27 & 22.97 & 22.51 & $\ldots$ \\
\hline 148 & 064133.49 & -505216.3 & 0.04502640 & 23.46 & 23.21 & $\ldots$ & $\ldots$ \\
\hline 149 & 064133.6 & -505317.2 & 0.06592061 & 23.15 & 22.84 & 22.50 & $\ldots$ \\
\hline 150 & 064133.68 & -510222.5 & 0.0607464 & 23.27 & 22.95 & 22.64 & $\ldots$ \\
\hline 151 & 064133.87 & -505752.6 & 0.05790728 & 23.09 & 22.83 & 22.43 & $\ldots$ \\
\hline 152 & 064134.1 & -510452.1 & 0.05515096 & 23.48 & 23.13 & 22.65 & $\ldots$ \\
\hline 153 & 064134.39 & -505944.7 & 0.08287027 & 22.66 & 22.33 & 21.90 & $\ldots$ \\
\hline 154 & 064134.74 & -505258.7 & 0.05223474 & 23.53 & 23.24 & $\ldots$ & $\ldots$ \\
\hline 155 & 064135.37 & -510757.2 & 0.05196965 & 23.55 & 23.25 & $\ldots$ & $\ldots$ \\
\hline 156 & 064135.62 & -505221.0 & 0.05518927 & 23.46 & 23.17 & 22.75 & $\ldots$ \\
\hline 157 & 064136.08 & -510031.2 & 0.06138969 & 23.57 & 23.26 & 22.64 & $\ldots$ \\
\hline 159 & 064136.81 & -505830.4 & 0.06345606 & 22.93 & 22.63 & 22.10 & $\ldots$ \\
\hline 160 & 064136.91 & -510546.8 & 0.794001 & 22.95 & 22.64 & 17.16 & $\ldots$ \\
\hline 161 & 064137.15 & -510726.0 & 0.06743787 & 23.32 & 22.98 & 22.26 & $\ldots$ \\
\hline 162 & 064137.44 & -505744.1 & 0.04950919 & 23.44 & 23.16 & $\ldots$ & $\ldots$ \\
\hline 163 & 064137.52 & -505554.8 & 0.05993709 & 23.45 & 23.14 & 22.58 & $\ldots$ \\
\hline 164 & 064137.61 & -505941.7 & 0.06221228 & 23.13 & 22.88 & 22.04 & $\ldots$ \\
\hline 165 & 064137.68 & -505546.8 & 0.05804816 & 23.26 & 23.00 & 22.46 & $\ldots$ \\
\hline 166 & 064137.77 & -505930.2 & 0.06451736 & 23.14 & 22.87 & 22.49 & $\ldots$ \\
\hline 167 & 064138.48 & -50577.7 & 0.0538934 & 23.33 & 23.04 & 22.46 & $\ldots$ \\
\hline 168 & 064138.71 & -510452.4 & 0.05157579 & 23.48 & 23.19 & $\ldots$ & $\ldots$ \\
\hline 169 & 064139.09 & -505052.8 & 0.0494872 & 23.37 & 23.12 & $\ldots$ & $\ldots$ \\
\hline 170 & 064139.15 & $\begin{array}{llll}-51 & 10 & 26.1\end{array}$ & 0.05545547 & 23.46 & 23.17 & $\ldots$ & $\ldots$ \\
\hline 171 & 064139.34 & -50530.5 & 0.05387548 & 23.17 & 22.88 & 22.54 & $\ldots$ \\
\hline 172 & 064139.47 & -505848.9 & 0.07324209 & 22.99 & 22.66 & 22.19 & $\ldots$ \\
\hline 173 & 064139.55 & -504743.3 & 0.05155752 & 23.36 & 23.03 & $\ldots$ & $\ldots$ \\
\hline 174 & 064140.39 & -505439.7 & 0.06174584 & 23.42 & 23.12 & $\ldots$ & $\ldots$ \\
\hline 175 & 064140.54 & -510058.8 & 0.05422135 & 23.43 & 23.18 & $\ldots$ & $\ldots$ \\
\hline 176 & 064140.59 & -505911.0 & 0.05880960 & 23.50 & 23.22 & $\ldots$ & $\ldots$ \\
\hline 177 & 064140.66 & -504633.7 & 0.05548850 & 23.36 & 23.03 & $\ldots$ & $\ldots$ \\
\hline 178 & 064141.01 & -504845.8 & 0.05676424 & 23.42 & 23.16 & $\ldots$ & $\ldots$ \\
\hline 179 & 064141.45 & -510355.3 & 0.05777849 & 23.31 & 22.98 & 22.50 & $\ldots$ \\
\hline 181 & 064141.67 & -505550.3 & 0.05891665 & 23.40 & 23.07 & 22.53 & $\ldots$ \\
\hline 182 & 064142.16 & -510350.8 & 0.06129068 & 23.43 & 23.12 & 22.69 & $\ldots$ \\
\hline 183 & 064142.21 & -505712.3 & 0.10774755 & 22.40 & 22.00 & 21.51 & $\ldots$ \\
\hline 184 & 064143.02 & $\begin{array}{lll}-51 & 02 & 27.1\end{array}$ & 0.05879365 & 22.89 & 22.59 & 22.17 & $\ldots$ \\
\hline 185 & 064143.11 & -51018.2 & 0.04519765 & 23.02 & 22.82 & 22.59 & $\ldots$ \\
\hline 186 & 064143.19 & -510446.4 & 0.06336417 & 23.00 & 22.69 & 22.27 & $\ldots$ \\
\hline 187 & 064143.51 & $\begin{array}{llll}-51 & 04 & 12.0\end{array}$ & 0.05053246 & 23.45 & 23.18 & 22.75 & $\ldots$ \\
\hline 188 & 064143.56 & -505246.5 & 0.06248493 & 23.08 & 22.77 & 22.30 & $\ldots$ \\
\hline 189 & 064144.16 & -510424.9 & 0.06737899 & 23.19 & 22.90 & 22.50 & $\ldots$ \\
\hline 190 & 064144.58 & -510316.4 & 0.05927821 & 22.93 & 22.65 & 22.24 & $\ldots$ \\
\hline 191 & 064144.66 & $\begin{array}{lll}-51 & 09 & 12.9\end{array}$ & 0.05014270 & 23.43 & 23.15 & $\ldots$ & $\ldots$ \\
\hline 192 & 064144.69 & $\begin{array}{lll}-51 & 02 & 44.2\end{array}$ & 0.05215024 & 23.23 & 22.95 & 22.51 & $\ldots$ \\
\hline 193 & 064144.72 & -505013.0 & 0.06786510 & 23.29 & 22.99 & 22.62 & $\ldots$ \\
\hline 194 & 064144.96 & -50545.7 & 0.0638021 & 23.25 & 22.94 & 22.52 & $\ldots$ \\
\hline 195 & 064145.1 & -505611.4 & 0.0604508 & 23.28 & 23.00 & 22.45 & $\ldots$ \\
\hline 196 & 064145.53 & $\begin{array}{lll}-51 & 01 & 35.4\end{array}$ & 0.05485589 & 23.39 & 23.11 & 22.66 & $\ldots$ \\
\hline 197 & 064145.64 & -510521.8 & 0.05700529 & 23.46 & 23.19 & 22.58 & $\ldots$ \\
\hline 198 & 064146.01 & -510422.6 & 0.0552781 & 23.37 & 23.14 & 22.65 & $\ldots$ \\
\hline 199 & 064146.0 & -50530.7 & 0.05127621 & 23.47 & 23.15 & 22.72 & $\ldots$ \\
\hline 200 & 064146.11 & -50508.1 & 0.05729547 & 22.78 & 22.20 & 21.40 & $\ldots$ \\
\hline 201 & 064146.16 & -511235.1 & 0.05264742 & 23.01 & 22.76 & 22.41 & $\ldots$ \\
\hline 202 & 064146.47 & -505510.8 & 0.06236390 & 23.18 & 22.92 & 22.52 & V20 \\
\hline 203 & 064146.62 & -505243.8 & 0.05846323 & 23.16 & 22.94 & 22.64 & $\ldots$ \\
\hline
\end{tabular}


Table 13

(Continued)

\begin{tabular}{|c|c|c|c|c|c|c|c|}
\hline ID & $\alpha$ & $\delta$ & Period (day) & $\langle B\rangle^{b}$ & $\langle V\rangle^{b}$ & $\langle I\rangle^{b}$ & M98 \\
\hline 204 & 064146.72 & -505827.3 & 0.05327753 & 23.47 & 23.22 & $\ldots$ & $\ldots$ \\
\hline 205 & 064146.83 & $\begin{array}{llll}-51 & 05 & 2.3\end{array}$ & 0.05975768 & 23.37 & 23.04 & 22.65 & $\ldots$ \\
\hline 206 & 064146.94 & -505852.0 & 0.05747476 & 23.42 & 23.15 & 22.46 & $\ldots$ \\
\hline 207 & 064147.16 & -505330.2 & 0.06124722 & 22.89 & 22.59 & 22.09 & $\ldots$ \\
\hline 208 & 064147.17 & -50598.2 & 0.05641026 & 22.63 & 22.37 & 21.94 & $\ldots$ \\
\hline 209 & 064147.38 & -505127.5 & 0.05379832 & 23.34 & 23.05 & 22.62 & $\ldots$ \\
\hline 210 & 064147.4 & -504931.8 & 0.06601826 & 23.22 & 22.95 & 22.67 & $\ldots$ \\
\hline 211 & 064147.49 & -505447.0 & 0.03863078 & 23.40 & 23.09 & 22.65 & $\ldots$ \\
\hline 212 & 064147.55 & -510048.9 & 0.05764756 & 23.32 & 23.04 & 22.58 & $\ldots$ \\
\hline 213 & 064147.69 & -50549.8 & 0.06027106 & 23.43 & 23.12 & 22.65 & $\ldots$ \\
\hline 214 & 064147.72 & -50523.6 & 0.04701230 & 23.48 & 23.22 & 22.78 & $\ldots$ \\
\hline 215 & 064147.8 & -510136.8 & 0.05662963 & 23.36 & 23.10 & 22.81 & $\ldots$ \\
\hline 216 & 064148.37 & -505055.6 & 0.05979195 & 23.32 & 23.03 & 22.61 & $\ldots$ \\
\hline 217 & 064149.31 & -505757.6 & 0.05807511 & 23.48 & 23.16 & 22.59 & $\ldots$ \\
\hline 218 & 064149.96 & -50465.0 & 0.06366226 & 23.17 & 22.94 & 22.58 & $\ldots$ \\
\hline 219 & 064150.43 & $\begin{array}{lll}-51 & 04 & 14.3\end{array}$ & 0.06075013 & 23.34 & 23.08 & 22.60 & $\ldots$ \\
\hline 220 & 064150.66 & -510049.5 & 0.05904003 & 23.21 & 22.90 & 22.51 & $\ldots$ \\
\hline 221 & 064150.79 & -505853.0 & 0.05209704 & 23.37 & 23.08 & 22.65 & $\ldots$ \\
\hline 222 & 064151.04 & -505429.0 & 0.05576081 & 23.41 & 23.11 & 22.66 & $\ldots$ \\
\hline 223 & 064151.06 & --510431.7 & 0.06984379 & 23.21 & 22.91 & 22.38 & $\ldots$ \\
\hline 224 & 064151.68 & -505727.0 & 0.05308256 & 23.38 & 23.07 & 22.61 & $\ldots$ \\
\hline 225 & 064151.7 & -50574.3 & 0.07427555 & 23.23 & 22.89 & 22.46 & $\ldots$ \\
\hline 226 & 064151.89 & -51041.4 & 0.05877806 & 22.95 & 22.68 & 22.24 & $\ldots$ \\
\hline 227 & 064151.94 & -510246.5 & 0.04577285 & 23.06 & 22.85 & 22.56 & $\ldots$ \\
\hline 228 & 064152.29 & -50587.4 & 0.05670511 & 23.31 & 23.10 & 22.67 & $\ldots$ \\
\hline 229 & 064152.74 & -505650.2 & 0.05704030 & 23.32 & 22.98 & 22.56 & $\ldots$ \\
\hline 230 & 064152.78 & -505912.5 & 0.05804334 & 23.58 & 23.28 & $\ldots$ & $\ldots$ \\
\hline 231 & 064152.83 & -51048.9 & 0.06504728 & 23.24 & 22.96 & 22.56 & $\ldots$ \\
\hline 232 & 064153.2 & -504630.9 & 0.06587775 & 23.34 & 23.01 & 22.59 & $\ldots$ \\
\hline 233 & 064153.2 & -505515.6 & 0.05519357 & 22.88 & 22.55 & 22.08 & $\ldots$ \\
\hline 234 & 064153.3 & -510022.5 & 0.07879928 & 23.04 & 22.76 & 22.34 & $\ldots$ \\
\hline 235 & 064154.04 & -505435.2 & 0.05946258 & 23.44 & 23.13 & 22.60 & $\ldots$ \\
\hline 236 & 064154.11 & -510611.7 & 0.05388561 & 23.38 & 23.08 & 22.61 & $\ldots$ \\
\hline 237 & 064154.81 & -510244.2 & 0.06376287 & 23.33 & 23.01 & 22.57 & $\ldots$ \\
\hline 238 & 064155.75 & -505828.9 & 0.05211579 & 23.42 & 23.18 & $\ldots$ & $\ldots$ \\
\hline 239 & 064156.05 & -504821.0 & 0.05420404 & 23.49 & 23.23 & 22.69 & $\ldots$ \\
\hline 240 & 064156.9 & -50587.1 & 0.06098996 & 23.24 & 22.96 & 22.47 & $\ldots$ \\
\hline 241 & 064156.93 & -510610.7 & 0.06707216 & 23.29 & 22.97 & 22.49 & $\ldots$ \\
\hline 242 & 064157.13 & -505611.8 & 0.05633799 & 22.82 & 22.52 & 22.11 & V18 \\
\hline 243 & 064157.35 & -50533.3 & 0.05820235 & 23.45 & 23.14 & 22.69 & $\ldots$ \\
\hline 244 & 064157.71 & -505422.9 & 0.03887685 & 23.28 & 22.98 & 22.63 & $\ldots$ \\
\hline 245 & 064158.42 & -510546.3 & 0.06735249 & 23.27 & 22.96 & 22.50 & $\ldots$ \\
\hline 246 & 064158.93 & -510135.5 & 0.06902505 & 23.13 & 22.82 & 22.33 & $\ldots$ \\
\hline 247 & 064159.06 & -505614.1 & 0.07260680 & 23.19 & 22.89 & 22.44 & $\ldots$ \\
\hline 248 & 064159.51 & -50497.0 & 0.05233172 & 23.48 & 23.18 & $\ldots$ & $\ldots$ \\
\hline 249 & 064159.69 & -504523.6 & 0.07625333 & 23.09 & 22.79 & 22.32 & $\ldots$ \\
\hline 250 & 06420.03 & -505643.3 & 0.07485741 & 23.16 & 22.83 & 22.44 & $\ldots$ \\
\hline 251 & 06420.05 & -505552.2 & 0.06031459 & 23.16 & 22.90 & 22.57 & V17 \\
\hline 252 & 06420.42 & -505728.4 & 0.05574277 & 23.21 & 22.96 & $\ldots$ & $\ldots$ \\
\hline 253 & 06420.61 & -510138.8 & 0.05781329 & 23.53 & 23.23 & 22.78 & $\ldots$ \\
\hline 254 & 06420.97 & -504612.8 & 0.07540239 & 22.73 & 22.42 & 22.00 & $\ldots$ \\
\hline 255 & 06420.97 & -510520.9 & 0.05291626 & 23.51 & 23.19 & $\ldots$ & $\ldots$ \\
\hline 256 & 06421.11 & -505331.3 & 0.05283800 & 23.51 & 23.21 & 22.74 & $\ldots$ \\
\hline 257 & 06421.31 & -510124.7 & 0.05176433 & 23.46 & 23.19 & 22.72 & $\ldots$ \\
\hline 258 & 06421.72 & -510122.7 & 0.05421737 & 23.26 & 23.01 & 22.57 & $\ldots$ \\
\hline 259 & 06421.83 & -505152.9 & 0.06569699 & 23.26 & 22.96 & 22.53 & $\ldots$ \\
\hline 260 & 06421.88 & -505551.3 & 0.05638275 & 22.98 & 22.68 & 22.28 & $\ldots$ \\
\hline 261 & 06421.97 & -504946.9 & 0.05943899 & 23.30 & 23.02 & 22.65 & $\ldots$ \\
\hline 262 & 06422.65 & -50587.1 & 0.05790880 & 23.17 & 22.92 & 22.38 & $\ldots$ \\
\hline 263 & 06423.21 & -50534.3 & 0.06510705 & 23.36 & 23.01 & 22.54 & $\ldots$ \\
\hline 265 & 06423.72 & -505340.1 & 0.06100386 & 23.34 & 23.04 & 22.59 & $\ldots$ \\
\hline 266 & 06423.84 & -505255.2 & 0.05297954 & 23.39 & 23.11 & 22.62 & $\ldots$ \\
\hline 267 & 06424.06 & -505543.7 & 0.05502621 & 23.42 & 23.11 & 22.69 & $\ldots$ \\
\hline 268 & 06424.51 & -50550.6 & 0.05722660 & 22.88 & 22.59 & 22.19 & $\ldots$ \\
\hline
\end{tabular}


Table 13

(Continued)

\begin{tabular}{|c|c|c|c|c|c|c|c|}
\hline ID & $\alpha$ & $\delta$ & Period (day) & $\langle B\rangle^{b}$ & $\langle V\rangle^{b}$ & $\langle I\rangle^{b}$ & M98 \\
\hline 269 & 06424.95 & $\begin{array}{llll}-51 & 05 & 9.3\end{array}$ & 0.06504377 & 23.44 & 23.12 & 22.63 & $\ldots$ \\
\hline 270 & 06425.03 & -505850.3 & 0.05499679 & 23.53 & 23.23 & 22.72 & $\ldots$ \\
\hline 271 & 06425.26 & -505557.4 & 0.06152380 & 23.18 & 22.86 & 22.39 & $\ldots$ \\
\hline 272 & 06425.28 & -50492.3 & 0.05880554 & 23.36 & 23.07 & 22.59 & $\ldots$ \\
\hline 273 & 06425.55 & -510257.8 & 0.05339638 & 23.43 & 23.15 & 22.68 & $\ldots$ \\
\hline 274 & 06425.61 & -505513.2 & 0.04986932 & 23.47 & 23.20 & 22.73 & $\ldots$ \\
\hline 275 & 06425.87 & $\begin{array}{lll}-51 & 01 & 40.2\end{array}$ & 0.05530886 & 23.29 & 23.03 & 22.60 & $\ldots$ \\
\hline 276 & 06426.14 & -505633.9 & 0.05151944 & 23.25 & 22.95 & 22.52 & $\ldots$ \\
\hline 277 & 06426.21 & -505731.3 & 0.05929224 & 23.47 & 23.14 & 22.28 & $\ldots$ \\
\hline 278 & 06426.24 & -50469.9 & 0.05903120 & 23.20 & 22.91 & 22.48 & $\ldots$ \\
\hline 279 & 06427.06 & -505913.3 & 0.0689945 & 23.20 & 22.91 & 22.50 & $\ldots$ \\
\hline 280 & 06427.26 & -510346.8 & 0.05790894 & 22.71 & 22.50 & 22.14 & $\ldots$ \\
\hline 281 & 06427.44 & -505620.0 & 0.05449367 & 22.73 & 22.44 & 22.08 & $\ldots$ \\
\hline 282 & 06427.78 & -511215.5 & 0.0677668 & 23.10 & 22.79 & 22.41 & $\ldots$ \\
\hline 283 & 06428.17 & -51062.3 & 0.06326881 & 22.71 & 22.40 & 22.04 & $\ldots$ \\
\hline 285 & 06428.95 & -504949.7 & 0.05939892 & 23.39 & 23.10 & 22.63 & $\ldots$ \\
\hline 286 & 06429.02 & -50479.5 & 0.06478860 & 23.17 & 22.91 & 22.49 & $\ldots$ \\
\hline 287 & 06429.79 & -505328.6 & 0.05328744 & 23.40 & 23.12 & 22.71 & $\ldots$ \\
\hline 288 & $0642 \quad 10.25$ & -504542.3 & 0.06449873 & 23.12 & 22.89 & 22.54 & $\ldots$ \\
\hline 289 & $0642 \quad 11.36$ & -510334.1 & 0.06686331 & 23.19 & 22.92 & 22.52 & $\ldots$ \\
\hline 290 & $0642 \quad 11.54$ & -505152.4 & 0.05631450 & 23.51 & 23.22 & 22.74 & $\ldots$ \\
\hline 291 & $0642 \quad 11.78$ & -505614.7 & 0.06001563 & 23.42 & 23.10 & 22.67 & $\ldots$ \\
\hline 292 & $0642 \quad 14.39$ & -510136.0 & 0.06529897 & 23.23 & 22.94 & 22.55 & $\ldots$ \\
\hline 293 & $0642 \quad 14.53$ & -504719.1 & 0.05494963 & 23.39 & 23.10 & 22.72 & $\ldots$ \\
\hline 294 & $0642 \quad 14.67$ & -50536.3 & 0.0590915 & 23.44 & 23.11 & 22.70 & $\ldots$ \\
\hline 296 & $0642 \quad 15.21$ & -505839.3 & 0.05625275 & 23.31 & 23.03 & 22.54 & $\ldots$ \\
\hline 297 & $0642 \quad 15.62$ & -50545.5 & 0.06991609 & 23.10 & 22.84 & 22.45 & $\ldots$ \\
\hline 298 & $0642 \quad 16.43$ & -50560.4 & 0.07426899 & 23.17 & 22.85 & 22.36 & $\ldots$ \\
\hline 299 & $0642 \quad 16.57$ & -505137.4 & 0.05705933 & 23.45 & 23.15 & 22.66 & $\cdots$ \\
\hline 300 & $0642 \quad 17.33$ & -505828.8 & 0.05419873 & 23.28 & 23.02 & 22.54 & $\ldots$ \\
\hline 301 & $0642 \quad 19.44$ & -505529.0 & 0.05615369 & 22.22 & 21.92 & 21.51 & $\ldots$ \\
\hline 302 & $0642 \quad 19.48$ & -504316.2 & 0.05671087 & 23.36 & 23.07 & 22.53 & $\ldots$ \\
\hline 303 & $0642 \quad 19.67$ & -505520.4 & 0.06518118 & 23.25 & 22.93 & 22.51 & $\ldots$ \\
\hline 304 & $0642 \quad 21.2$ & -50562.3 & 0.05414244 & 22.58 & 22.30 & 21.90 & $\ldots$ \\
\hline 305 & 064221.73 & -505215.0 & 0.05867896 & 23.16 & 22.89 & 22.43 & $\cdots$ \\
\hline 306 & 064223.36 & -510323.8 & 0.05754752 & 23.41 & 23.07 & 22.66 & $\ldots$ \\
\hline 307 & 064223.53 & -50492.5 & 0.04933231 & 23.32 & 23.13 & 22.58 & $\ldots$ \\
\hline 308 & $0642 \quad 25.71$ & -504437.8 & 0.05746768 & 23.19 & 22.97 & 22.66 & $\ldots$ \\
\hline 309 & $0642 \quad 26.22$ & -505441.8 & 0.05833121 & 23.34 & 23.08 & 22.71 & $\ldots$ \\
\hline 310 & $0642 \quad 30.21$ & -504740.6 & 0.06787074 & 23.12 & 22.86 & 22.43 & $\ldots$ \\
\hline 311 & 064230.6 & -504630.4 & 0.05826303 & 23.25 & 23.03 & 22.64 & $\ldots$ \\
\hline 312 & 064231.28 & -505612.1 & 0.07580626 & 23.19 & 22.88 & 22.38 & $\ldots$ \\
\hline 313 & 064231.83 & -505052.6 & 0.08140471 & 23.04 & 22.75 & 22.29 & $\ldots$ \\
\hline 314 & 064233.17 & -505637.5 & 0.05044822 & 23.47 & 23.23 & 22.70 & $\ldots$ \\
\hline 315 & 064234.36 & -505010.9 & 0.05782427 & 23.46 & 23.15 & 22.70 & $\ldots$ \\
\hline 316 & 064234.52 & -505256.2 & 0.05146992 & 23.19 & 22.90 & 22.55 & $\ldots$ \\
\hline 317 & 064239.61 & $\begin{array}{llll}-50 & 51 & 12.4\end{array}$ & 0.05268487 & 23.33 & 23.03 & 22.62 & $\ldots$ \\
\hline 318 & 064240.33 & -50556.3 & 0.05775708 & 23.50 & 23.21 & 22.72 & $\ldots$ \\
\hline 319 & 064240.36 & -505456.0 & 0.05554502 & 23.18 & 22.77 & 22.23 & $\ldots$ \\
\hline 320 & 064241.59 & -505742.3 & 0.06197303 & 22.93 & 22.62 & 22.19 & $\ldots$ \\
\hline 321 & 064243.39 & -504650.0 & 0.06700168 & 23.15 & 22.88 & 22.48 & $\ldots$ \\
\hline 322 & 064244.68 & -505524.5 & 0.04944784 & 23.42 & 23.16 & 22.69 & $\ldots$ \\
\hline 323 & 064245.69 & $\begin{array}{lll}-51 & 02 & 22.9\end{array}$ & 0.06264889 & 23.34 & 23.02 & 22.58 & $\cdots$ \\
\hline 324 & 064246.56 & -505622.7 & 0.05710797 & 23.27 & 23.01 & 22.59 & $\ldots$ \\
\hline 325 & 064246.79 & -503423.2 & 0.058553866 & 23.55 & 23.22 & $\ldots$ & $\ldots$ \\
\hline 326 & 064249.08 & -504454.3 & 0.06133020 & 22.88 & 22.59 & 22.17 & $\ldots$ \\
\hline 327 & 064254.51 & -50488.1 & 0.06121390 & 22.92 & 22.64 & 22.27 & $\ldots$ \\
\hline 328 & 064254.6 & -505911.1 & 0.05483696 & 22.96 & 22.68 & 22.26 & $\ldots$ \\
\hline 329 & 064255.87 & -50597.1 & 0.05684852 & 23.38 & 23.10 & 22.58 & $\ldots$ \\
\hline 330 & 06434.94 & -50563.9 & 0.06522519 & 23.12 & 22.79 & 22.33 & $\ldots$ \\
\hline 331 & $0643 \quad 10.89$ & -510342.2 & 0.05529448 & 23.39 & 23.07 & 22.67 & $\ldots$ \\
\hline 332 & $0643 \quad 18.68$ & -504819.4 & 0.05442560 & 23.07 & 22.80 & 22.42 & $\ldots$ \\
\hline 333 & 064322.28 & -505417.1 & 0.06204207 & 23.32 & 23.06 & 22.52 & $\ldots$ \\
\hline 334 & 064327.85 & -504551.3 & 0.05501861 & 23.30 & 23.04 & 22.63 & $\ldots$ \\
\hline
\end{tabular}


Table 13

(Continued)

\begin{tabular}{|c|c|c|c|c|c|c|c|}
\hline ID & $\alpha$ & $\delta$ & Period (day) & $\langle B\rangle^{b}$ & $\langle V\rangle^{b}$ & $\langle I\rangle^{b}$ & M98 \\
\hline 335 & 064338.45 & -505811.4 & 0.06575684 & 23.38 & 23.02 & $\ldots$ & $\ldots$ \\
\hline 336 & 064340.55 & -510228.6 & 0.05646906 & 23.45 & 23.18 & $\ldots$ & $\ldots$ \\
\hline 337 & 064351.08 & $\begin{array}{llll}-50 & 50 & 27.7\end{array}$ & 0.05884652 & 23.46 & 23.16 & $\ldots$ & \\
\hline$\ldots$ & 064102.39 & -510153.3 & 0.06397398 & 23.32 & 23.02 & 22.54 & V1 \\
\hline$\ldots$ & 064109.57 & -510120.6 & 0.04791289 & 23.46 & 23.15 & 22.73 & V2 \\
\hline$\ldots$ & 064104.12 & $\begin{array}{lll}-51 & 01 & 14.7\end{array}$ & 0.07691745 & 23.19 & 22.88 & 22.40 & V3 \\
\hline$\ldots$ & 064056.40 & -505939.8 & 0.05575196 & 23.50 & 23.14 & 22.79 & V8 \\
\hline$\ldots$ & 064102.22 & -505854.7 & 0.07171579 & 23.09 & 22.77 & 22.36 & V11 \\
\hline$\ldots$ & 064156.74 & -505613.7 & 0.05907561 & 23.08 & 22.78 & 22.35 & V18 \\
\hline$\ldots$ & 064155.20 & -505615.4 & 0.06491275 & 22.86 & 22.58 & 22.17 & V19 \\
\hline
\end{tabular}

Monelli was supported by the Education and Science Ministry of Spain (grants AYA2010-16717). M. Fabrizio acknowledges financial support from the PO FSE Abruzzo 2007-2013 through the grant "Spectro-photometric characterization of stellar populations in Local Group dwarf galaxies, prot.89/2014/OACTe/D (PI: S. Cassisi). G. Fiorentino was supported by the Futuro in Ricerca 2013 (RBFR13J716).

\section{APPENDIX \\ NOTES ON INDIVIDUAL VARIABLES}

V11, V26, V74: Classified as first-overtone pulsators by S86, according to the current photometry they seem to be doublemode pulsators.

V17: Classified as a suspected variable in S86 and NV by D03. According to the current photometry it appears to be an EB with a period of 0.3933393 days.

V22: Classified as first-overtone pulsator by S86 and D03. According to the current photometry it seems to pulsate in the fundamental mode with period of days.

V25, V31: Classified as first-overtone pulsators by $\mathrm{S} 86$. According to the current photometry they seem to pulsate in the fundamental mode with periods of 0.05765942 and 0.06037914 days and they also show the Blazhko effect.

V27, V29, V33, V129 and V149: Classified as fundamental mode pulsators by S86. According to the current photometry they appear to be ACs.

V40 and V115: Classified as uncertain variables by S86. According to our current set of data, they appear to pulsate as first-overtones and ACs, respectively.

V41 and V87: Classified as suspected variables by S86. According to our current set of data, they appear to be anomalous cepheids.

V61, V77, V126, V127: Classified as fundamental mode pulsators by S86. According to the current photometry they also show the Blazhko effect.

V89: Classified as a fundamental mode pulsator by S86. According to the current photometry it seems to be a doublemode pulsator.

V138 and V141: Classified as suspected variables by S86. According to our current set of data, they appear to be fundamental-mode pulsators.

V142 and V151: Classified as suspected variables by S86. According to our current set of data, they appear to be first overtone pulsators.
V176: Classified as a first-overtone pulsator by D03 with period of $\sim 0.4$. According to the current photometry it seems to pulsate in the fundamental mode with period of 0.764565 days.

V40, V65, V73, V84, V85, V123, V136, V138, V149, V177, V183, V186, V188, V195, V199, V201, V208, V211, V227, $V 228, V 229, V 230$ : The I-band light curves are poorly sampled in the pulsation across maximum light.

V31, V61, V76, V77, V126, V127, V206: Classified as fundamental pulsators by D03. According to the current photometry they seem to pulsate in the fundamental mode and they also show the Blazhko effect.

V74: Classified as a first overtone pulsator by D03. According to the current photometry they seem to pulsate in the double mode.

V85, V90, V181, V186, V196, V226: The light curves are noisy.

V148: This $\mathrm{RR}_{c}$ is $\sim 0.3 \mathrm{mag}$ brighter than the typical luminosity of $\mathrm{HB}$ stars.

V158, V182: We confirm the peculiar nature of the RRLs (see also Paper VI).

V161: Classified as a suspected variable in D03. According to the current photometry it does not show variability.

V170 and V171: Not enough data to fit light curves; uncertain parameters.

V176, V179, V196, V182, V200, V223: These RRLs have very small luminosity amplitudes for an ab-type RRL.

V181: This variable has a very small luminosity amplitude for a c-type RRL. Possible blend with nearby faint star.

V193, V205, V216 , V217, V219: These variables have very small amplitudes for an AC-type variable. Possible blends with nearby faint stars.

V3, V25, V44, V58, and V133: These stars were classified as newly discovered variables in Paper VI with the identification numbers: V215, V213, V212, V211, and V209, respectively.

V4, V32, V41 and V165: These stars were classified in D03 and Paper VI as V177, V202, V180 and V165, respectively.

V218, V227, V228, V229, V230: Poorly sampled light curves, pulsational parameters are uncertain.

$R R L-1, R R L-2, R R L-3, R R L-4, R R L-5, R R L-38, A C-1, A C-9$, $A C$-10: These stars are the six RRLs (three $\mathrm{RR}_{c}$, three $\mathrm{RR}_{a b}$ ) and the three ACs recently detected by VM13 outside the tidal radius of Carina. 


\section{REFERENCES}

Baldry, I. K., Driver, S. P., Loveday, J., et al. 2012, MNRAS, 421, 621

Bauer, A. E., Conselice, C. J., Pérez-González, P. G., et al. 2011, MNRAS, 417, 289

Benedict, G. F., McArthur, B. E., Feast, M. W., et al. 2011, AJ, 142, 187

Bessell, M. S. 1969, ApJS, 18, 195

Blanco-Cuaresma, S., Soubiran, C., Heiter, U., et al. 2015, A\&A, 577, A47

Bono, G., Caputo, F., Cassisi, S., Incerpi, R., \& Marconi, M. 1997a, ApJ, 483,811

Bono, G., Caputo, F., Castellani, V., \& Marconi, M. 1997b, A\&AS, 121, 327

Bono, G., Caputo, F., \& di Criscienzo, M. 2007, A\&A, 476, 779

Bono, G., Caputo, F., \& Marconi, M. 1995, AJ, 110, 2365

Bono, G., Dall'Ora, M., Caputo, F., et al. 2011, in RR Lyrae Stars, Metal-Poor Stars, and the Galaxy, ed. A. McWilliam (Pasadena, CA: The Carnegie Institution), 1

Bono, G., Marconi, M., Cassisi, S., et al. 2005, ApJ, 621, 966

Bono, G., Stetson, P. B., Walker, A. R., et al. 2010, PASP, 122, 651

Braga, V. F., Dall'Ora, M., Bono, G., et al. 2015, ApJ, 799, 165

Bragaglia, A., Gratton, R. G., Carretta, E., et al. 2001, AJ, 122, 207

Cacciari, C., Corwin, T. M., \& Carney, B. W. 2005, AJ, 129, 267

Caputo, F. 1998, A\&ARv, 9, 33

Cardelli, J. A., Clayton, G. C., \& Mathis, J. S. 1989, ApJ, 345, 245

Carretta, E., Bragaglia, A., Gratton, R., D’Orazi, V., \& Lucatello, S. 2009, A\&A, 508, 695

Carretta, E., Bragaglia, A., Gratton, R. G., et al. 2010, A\&A, 520, AA95

Castellani, V., Chieffi, A., \& Pulone, L. 1991, ApJS, 76, 911

Castellani, V., \& degl'Innocenti, S. 1995, A\&A, 298, 827

Clement, C. M., Ferance, S., \& Simon, N. R. 1993, ApJ, 412, 183

Clement, C. M., Kinman, T. D., \& Suntzeff, N. B. 1991, ApJ, 372, 273

Clement, C. M., \& Shelton, I. 1999, ApJL, 515, L85

Clementini, G., Cignoni, M., Contreras Ramos, R., et al. 2012, ApJ, 756, 108

Clementini, G., Corwin, T. M., Carney, B. W., \& Sumerel, A. N. 2004, AJ, 127,938

Clementini, G., Di Tomaso, S., di Fabrizio, L., et al. 2000, AJ, 120, 2054

Cole, A. A., Skillman, E. D., Tolstoy, E., et al. 2007, ApJL, 659, L17

Coppola, G., Stetson, P. B., Marconi, M., et al. 2013, ApJ, 775, 6 (Paper VI)

Cox, A. N., Hodson, S. W., \& Clancy, S. P. 1983, ApJ, 266, 94

Cseresnjes, P. 2001, A\&A, 375, 909

Da Costa, G. S., \& Hatzidimitriou, D. 1998, AJ, 115, 1934

Dalessandro, E., Ferraro, F. R., Massari, D., et al. 2013, ApJ, 778, 135

Dall'Ora, M., Clementini, G., Kinemuchi, K., et al. 2006, ApJL, 653, L109

Dall'Ora, M., Kinemuchi, K., Ripepi, V., et al. 2012, ApJ, 752, 42

Dall'Ora, M., Ripepi, V., Caputo, F., et al. 2003, AJ, 126, 197 (D03)

Di Criscienzo, M., Greco, C., Ripepi, V., et al. 2011, AJ, 141, 81

Di Criscienzo, M., Marconi, M., \& Caputo, F. 2004, ApJ, 612, 1092

Drake, A. J., Djorgovski, S. G., Mahabal, A., et al. 2009, ApJ, 696, 870

Duncan, K., Conselice, C. J., Mortlock, A., et al. 2014, MNRAS, 444, 2960

Eggen, O. J. 1979, ApJS, 41, 413

Eyer, L., Palaversa, L., Mowlavi, N., et al. 2012, Ap\&SS, 341, 207

Fabrizio, M., Nonino, M., Bono, G., et al. 2015, arXiv:1505.06597

Fabrizio, M., Raimondo, G., Brocato, E., et al. 2014, A\&A, 570, AA61

Fiorentino, G., Bono, G., Monelli, M., et al. 2015a, ApJL, 798, L12

Fiorentino, G., Bono, G., Monelli, M., et al. 2015b, ApJ, 810, 15

Fiorentino, G., Lanzoni, B., Dalessandro, E., et al. 2014, ApJ, 783, 34

Fiorentino, G., Limongi, M., Caputo, F., \& Marconi, M. 2006, A\&A, 460, 155

Fiorentino, G., Musella, I., \& Marconi, M. 2013, MNRAS, 434, 2866

Fiorentino, G., Stetson, P. B., Monelli, M., et al. 2012, ApJL, 759, L12

Gallart, C., Zoccali, M., \& Aparicio, A. 2005, ARA\&A, 43, 387

Garcia-Melendo, E., \& Clement, C. M. 1997, AJ, 114, 1190

Garofalo, A., Cusano, F., Clementini, G., et al. 2013, ApJ, 767, 62

Gilliland, R. L., Bono, G., Edmonds, P. D., et al. 1998, ApJ, 507, 818

Gratton, R. G., Bragaglia, A., Clementini, G., et al. 2004, A\&A, 421, 937

Greco, C., Dall'Ora, M., Clementini, G., et al. 2008, ApJL, 675, L73

Haschke, R., Grebel, E. K., Frebel, A., et al. 2012, AJ, 144, 88

Hatzidimitriou, D., Papadakis, I., Croke, B. F. W., et al. 1999, AJ, 117, 3059

Hog, E., \& Petersen, J. O. 1997, A\&A, 323, 827

Kaluzny, J., \& Thompson, I. B. 2003, AJ, 125, 2534

Kirby, E. N., Cohen, J. G., Guhathakurta, P., et al. 2013, ApJ, 779, 102

Kovács, G. 2001, A\&A, 375, 469

Kuehn, C., Kinemuchi, K., Ripepi, V., et al. 2008, ApJL, 674, L81
Kunder, A., \& Chaboyer, B. 2008, AJ, 136, 2441

Kunder, A., Stetson, P. B., Cassisi, S., et al. 2013, AJ, 146, 119

Layden, A. C. 1994, AJ, 108, 1016

Madore, B. F. 1982, ApJ, 253, 575

Madore, B. F., Hoffman, D., Freedman, W. L., et al. 2013, ApJ, 776, 135

Marconi, M., Bono, G., Caputo, F., et al. 2011, ApJ, 738, 111

Marconi, M., Coppola, G., Bono, G., Braga, V., et al. 2015, ApJ, 808, 50

Marconi, M., Fiorentino, G., \& Caputo, F. 2004, A\&A, 417, 1101

Marconi, M., Ripepi, V., Alcalá, J. M., et al. 2000, A\&A, 355, L35

Marengo, M., Evans, N. R., Barmby, P., et al. 2010, ApJ, 709, 120

Mateo, M., Hurley-Keller, D., \& Nemec, J. 1998, AJ, 115, 1856

Matsunaga, N., Feast, M. W., Kawadu, T., et al. 2013, MNRAS, 429, 385

Matsunaga, N., Kawadu, T., Nishiyama, S., et al. 2011, Natur, 477, 188

McConnachie, A. W. 2012, AJ, 144, 4

McNamara, D. H. 1995, AJ, 109, 1751

McNamara, D. H. 2000, PASP, 112, 1096

McNamara, D. H. 2011, AJ, 142, 110

McNamara, D. H., \& Barnes, J. 2014, AJ, 147, 31

Miceli, A., Rest, A., Stubbs, C. W., et al. 2008, ApJ, 678, 865

Monelli, M., Bernard, E. J., Gallart, C., et al. 2012a, MNRAS, 422, 89

Monelli, M., Cassisi, S., Mapelli, M., et al. 2012b, ApJ, 744, 157

Monelli, M., Gallart, C., Hidalgo, S. L., et al. 2010a, ApJ, 722, 1864

Monelli, M., Hidalgo, S. L., Stetson, P. B., et al. 2010b, ApJ, 720, 1225

Monelli, M., Milone, A. P., Fabrizio, M., et al. 2014, ApJ, 796, 90

Monelli, M., Pulone, L., Corsi, C. E., et al. 2003, AJ, 126, 218

Moretti, M. I., Dall'Ora, M., Ripepi, V., et al. 2009, ApJL, 699, L125

Mortlock, A., Conselice, C. J., Hartley, W. G., et al. 2013, MNRAS, 433, 1185

Musella, I., Ripepi, V., Clementini, G., et al. 2009, ApJL, 695, L83

Musella, I., Ripepi, V., Marconi, M., et al. 2012, ApJ, 756, 121

Nemec, J., \& Mateo, M. 1990, in ASP Conf. Ser. 10, Evolution of the Universe of Galaxies, Vol. 10, ed. R. G. Kron (San Francisco, CA: ASP), 134

Nemec, J. M. 1985a, AJ, 90, 204

Nemec, J. M. 1985b, AJ, 90, 240

Niss, B. 1981, A\&A, 98, 415

Okamoto, S., Arimoto, N., Yamada, Y., \& Onodera, M. 2008, A\&A, 487, 103

Olech, A., Dziembowski, W. A., Pamyatnykh, A. A., et al. 2005, MNRAS, 363,40

Palaversa, L., Ivezić, Ž, Eyer, L., et al. 2013, AJ, 146, 101

Petersen, J. O. 1991, A\&A, 243, 426

Petersen, J. O., \& Christensen-Dalsgaard, J. 1999, A\&A, 352, 547

Pietrinferni, A., Cassisi, S., Salaris, M., \& Castelli, F. 2004, ApJ, 612, 168

Pietrzyński, G., Górski, M., Gieren, W., et al. 2009, AJ, 138, 459

Pojmanski, G. 2002, ACTAA, 52, 397

Romaniello, M., Primas, F., Mottini, M., et al. 2008, A\&A, 488, 731

Saha, A., Monet, D. G., \& Seitzer, P. 1986, AJ, 92, 302

Salvadori, S., Ferrara, A., Schneider, R., Scannapieco, E., \& Kawata, D. 2010, MNRAS, 401, L5

Sandage, A., Katem, B., \& Sandage, M. 1981, ApJS, 46, 41

Sanna, N., Bono, G., Stetson, P. B., et al. 2009, ApJL, 699, L84

Santolamazza, P., Marconi, M., Bono, G., et al. 2001, ApJ, 554, 1124

Smith, H. A. 2006, MmSAI, 77, 492

Soszyński, I., Dziembowski, W. A., Udalski, A., et al. 2011, ACTAA, 61, 1

Soszynski, I., Poleski, R., Udalski, A., et al. 2008, ACTAA, 58, 163

Soszyński, I., Udalski, A., Szymański, M. K., et al. 2009, ACTAA, 59, 1

Soszyñski, I., Udalski, A., Szymañski, M. K., et al. 2010, ACTAA, 60, 165

Soszyński, I., Udalski, A., Szymański, M. K., et al. 2014, ACTAA, 64, 177

Springel, V., White, S. D. M., Jenkins, A., et al. 2005, Natur, 435, 629

Stetson, P. B., Braga, V. F., Dall'Ora, M., et al. 2014a, PASP, 126, 521

Stetson, P. B., Fiorentino, G., Bono, G., et al. 2014b, PASP, 126, 616

van Albada, T. S., \& Baker, N. 1971, ApJ, 169, 311

van den Bergh, S. 1975, in Galaxies and the Universe, ed. A. Sandage,

M. Sandage, \& J. Kristian (Chicago, IL: Univ. of Chicago Press), 509

Vivas, A. K., \& Mateo, M. 2013, AJ, 146, 141 (VM13)

Vivas, A. K., Zinn, R., Abad, C., et al. 2004, AJ, 127, 1158

Walker, A. R. 1994, AJ, 108, 555

Walker, A. R., \& Nemec, J. M. 1996, AJ, 112, 2026

Walker, A. R., \& Terndrup, D. M. 1991, ApJ, 378, 119

Weinzirl, T., Jogee, S., Conselice, C. J., et al. 2011, ApJ, 743, 87

Zinn, R., \& West, M. J. 1984, ApJS, 55, 45

Zoccali, M., Hill, V., Lecureur, A., et al. 2008, A\&A, 486, 177 